ESAIM: COCV

Vol. 13, No 3,2007 , pp. 419-457

DOI: $10.1051 /$ cocv:2007036
ESAIM: Control, Optimisation and Calculus of Variations

www.edpsciences.org/cocv

\title{
JUNCTION OF ELASTIC PLATES AND BEAMS
}

\author{
Antonio Gaudiello ${ }^{1}$, RéGis Monneau ${ }^{2}$, Jacqueline Mossino $^{3}$, \\ FranÇOIS MURAT $^{4}$ AND Ali Sili ${ }^{5}$
}

\begin{abstract}
We consider the linearized elasticity system in a multidomain of $\mathbf{R}^{3}$. This multidomain is the union of a horizontal plate with fixed cross section and small thickness $\varepsilon$, and of a vertical beam with fixed height and small cross section of radius $r^{\varepsilon}$. The lateral boundary of the plate and the top of the beam are assumed to be clamped. When $\varepsilon$ and $r^{\varepsilon}$ tend to zero simultaneously, with $r^{\varepsilon} \gg \varepsilon^{2}$, we identify the limit problem. This limit problem involves six junction conditions.
\end{abstract}

Mathematics Subject Classification. 35B40, 74B05, 74K30.

Received July 14, 2005.

\section{INTRODUCTION}

Let $\omega^{a}$ and $\omega^{b}$ ( $a$ for "above", $b$ for "below") be two bounded regular domains in $\mathbf{R}^{2}$. In the whole paper, the origin and axes are chosen so that:

$$
\int_{\omega^{a}} x_{1} \mathrm{~d} x_{1} \mathrm{~d} x_{2}=\int_{\omega^{a}} x_{2} \mathrm{~d} x_{1} \mathrm{~d} x_{2}=\int_{\omega^{a}} x_{1} x_{2} \mathrm{~d} x_{1} \mathrm{~d} x_{2}=0 \quad \text { and } \quad 0 \in \omega^{b} .
$$

Let $\varepsilon$ be a parameter taking values in a sequence of positive numbers converging to zero, and let $r^{\varepsilon}$ be another positive parameter tending to zero with $\varepsilon$. We introduce the thin multidomain $\Omega^{\varepsilon}=\Omega^{a \varepsilon} \bigcup J^{\varepsilon} \bigcup \Omega^{b \varepsilon}$, where $\Omega^{a \varepsilon}=r^{\varepsilon} \omega^{a} \times(0,1)$ represents a vertical beam with fixed height and small cross section, $\Omega^{b \varepsilon}=\omega^{b} \times(-\varepsilon, 0)$ represents a horizontal plate with small thickness and fixed cross section, and $J^{\varepsilon}=r^{\varepsilon} \omega^{a} \times\{0\}$ represents the interface at the junction between the beam and the plate.

Keywords and phrases. Junctions, thin structures, plates, beams, linear elasticity, asymptotic analysis.

1 Dipartimento di Automazione, Elettromagnetismo, Ingegneria dell'Informazione e Matematica Industriale, Università di Cassino, via G. Di Biasio 43, 03043 Cassino (FR), Italia; gaudiell@unina.it

2 CERMICS, École Nationale des Ponts et Chaussées, 6 et 8 Avenue Blaise Pascal, Cité Descartes, 77455 Champs-sur-Marne Cedex 2, France; monneau@cermics.enpc.fr

3 CMLA, École Normale Supérieure de Cachan, 61 Avenue du Président Wilson, 94235 Cachan Cedex, France; mossino@cmla.ens-cachan.fr

4 Laboratoire Jacques-Louis Lions, Université Pierre et Marie Curie, Boîte courrier 187, 75252 Paris Cedex 05, France; murat@ann.jussieu.fr

5 Département de Mathématiques, Université de Toulon et du Var, BP 132, 83957 La Garde Cedex, France; sili@univ-tln.fr

(c) EDP Sciences, SMAI 2007 
In this thin multidomain, we consider the displacement $\bar{U}^{\varepsilon}$, solution of the three-dimensional linearized elasticity system:

$$
\left\{\begin{array}{l}
\bar{U}^{\varepsilon} \in Y^{\varepsilon} \text { and } \forall U \in Y^{\varepsilon} \\
\int_{\Omega^{\varepsilon}}\left[A^{\varepsilon} e\left(\bar{U}^{\varepsilon}\right), e(U)\right] \mathrm{d} x=\int_{\Omega^{\varepsilon}} F^{\varepsilon} \cdot U \mathrm{~d} x+\int_{\Omega^{\varepsilon}}\left[G^{\varepsilon}, e(U)\right] \mathrm{d} x+\int_{\Sigma^{a \varepsilon} \cup T^{b \varepsilon} \cup B^{b \varepsilon}} H^{\varepsilon} \cdot U \mathrm{~d} \sigma
\end{array}\right.
$$

where:

- $Y^{\varepsilon}=\left\{U \in\left(H^{1}\left(\Omega^{\varepsilon}\right)\right)^{3}, U=0\right.$ on $T^{a \varepsilon}=r^{\varepsilon} \omega^{a} \times\{1\}$ and on $\left.\Sigma^{b \varepsilon}=\partial \omega^{b} \times(-\varepsilon, 0)\right\}$,

- $A^{\varepsilon}=A^{\varepsilon}(x)=\left\{\begin{array}{c}A^{a}, \text { if } x \in \Omega^{a \varepsilon}, \\ k^{\varepsilon} A^{b}, \text { if } x \in \Omega^{b \varepsilon},\end{array}\right.$

with $k^{\varepsilon}$ a positive parameter depending on $\varepsilon$ and $A^{a}, A^{b}$ tensors with constant coefficients $A_{i j k l}^{a}$ and $A_{i j k l}^{b}$, $i, j, k, l \in\{1,2,3\}$, satisfying the usual symmetry and coercivity conditions:

$$
\begin{gathered}
A_{i j k l}^{a}=A_{j i k l}^{a}=A_{i j l k}^{a}, \quad A_{i j k l}^{b}=A_{j i k l}^{b}=A_{i j l k}^{b}, \\
\exists C>0, \forall \xi \in \mathbf{R}_{s}^{3 \times 3},\left[A^{a} \xi, \xi\right] \geq C|\xi|^{2},\left[A^{b} \xi, \xi\right] \geq C|\xi|^{2},
\end{gathered}
$$

where $\mathbf{R}_{s}^{3 \times 3}$ denotes the set of symmetric $3 \times 3$-matrices, $\left(A^{a} \xi\right)_{i j}=\sum_{k l} A_{i j k l}^{a} \xi_{k l}$, the scalar product [., .] in $\mathbf{R}^{3 \times 3}$ is defined by $[\eta, \xi]=\sum_{i j} \eta_{i j} \xi_{i j}$ and $|$.$| is the associated norm; the euclidian scalar product in \mathbf{R}^{3}$ is denoted by a dot;

- $e_{i j}(U)=\frac{1}{2}\left(\frac{\partial U_{i}}{\partial x_{j}}+\frac{\partial U_{j}}{\partial x_{i}}\right)$

- $F^{\varepsilon} \in\left(L^{2}\left(\Omega^{\varepsilon}\right)\right)^{3}$,

- $G^{\varepsilon} \in\left(L^{2}\left(\Omega^{\varepsilon}\right)\right)^{3 \times 3}$,

- $H^{\varepsilon} \in\left(L^{2}\left(\Sigma^{a \varepsilon} \cup T^{b \varepsilon} \cup B^{b \varepsilon}\right)\right)^{3}$, where $\Sigma^{a \varepsilon}$ denotes the lateral boundary of the beam, $T^{b \varepsilon}$ and $B^{b \varepsilon}$ are respectively the top and the bottom of the plate:

$$
\Sigma^{a \varepsilon}=r^{\varepsilon} \partial \omega^{a} \times(0,1), \quad T^{b \varepsilon}=\left(\omega^{b} \backslash r^{\varepsilon} \omega^{a}\right) \times\{0\}, \quad B^{b \varepsilon}=\omega^{b} \times\{-\varepsilon\} .
$$

The constraint " $U=0$ " in the definition of $Y^{\varepsilon}$ means that the multistructure is clamped on the top $T^{a \varepsilon}$ of the beam and on the lateral boundary $\Sigma^{b \varepsilon}$ of the plate. The case $k^{\varepsilon}$ tending to zero or infinity corresponds to very different materials in $\Omega^{a \varepsilon}$ and $\Omega^{b \varepsilon}$ (note that breaking the symmetry between $\Omega^{a \varepsilon}$ and $\Omega^{b \varepsilon}$ by introducing the coefficient $k^{\varepsilon}$ in front of $A^{b}$ is not restrictive). In the right-hand side of (1.2), the second term is written in divergence form like in $[15,27,28]$. It is well known that, by means of the Green formula, this second term can contribute to the other ones, giving possibly less regular (not necessarily $L^{2}$ ) volume and surface source terms. For convenience of the reader, we have chosen to write the three integrals: one recovers the classical formulation by setting $G^{\varepsilon}=0$, but the simplest case corresponds to $F^{\varepsilon}=0, H^{\varepsilon}=0$ and $G^{\varepsilon} \neq 0$. This case was considered in the short preliminary version [15].

Problem (1.2) admits a unique solution $\bar{U}^{\varepsilon}$ (see e.g. [29]). The aim of this paper is to describe the limit behaviour of the displacement $\bar{U}^{\varepsilon}$, as $\varepsilon$ tends to zero. We prove that this behaviour depends on the limit of the sequence $q^{\varepsilon}$ defined by:

$$
q^{\varepsilon}=k^{\varepsilon} \frac{\varepsilon^{3}}{\left(r^{\varepsilon}\right)^{2}}
$$


When $k^{\varepsilon} \varepsilon^{3}$ and $\left(r^{\varepsilon}\right)^{2}$ have same order (i.e. when $q^{\varepsilon}$ tends to $q$ with $0<q<+\infty$ ), the limit problem (obtained after suitable rescaling) is a coupled problem between a two-dimensional plate and a one-dimensional beam, with six junction conditions. If $k^{\varepsilon} \varepsilon^{3} \gg\left(r^{\varepsilon}\right)^{2}$, the multistructure has the limit behaviour of a thin rigid plate and a thin elastic beam which are independent of each other, the beam being clamped at both ends; on the contrary, if $k^{\varepsilon} \varepsilon^{3} \ll\left(r^{\varepsilon}\right)^{2}$, the structure behaves as a thin rigid beam and a thin elastic plate which are independent of each other, the plate being clamped on its contour and fixed vertically at the junction.

The reader is referred to $[1,3,4,6-8,10-12,21-23,25-28,30,31]$ for the derivation of the equations of plates and beams by asymptotic analysis. Junction problems are considered in $[5,9,13,14,16-20]$. The present work is a natural follow up of $[27,28]$, which deal with reduction of dimension for elastic thin cylinders, and of $[13,14]$, which deal with the diffusion equation in the thin multistructure considered in this paper. Our results were announced in the short note [15].

\section{THE RESUlT}

\subsection{The rescaled problem}

In the sequel, the indexes $\alpha$ and $\beta$ take values in the set $\{1,2\}$. Moreover, $x=\left(x^{\prime}, x_{3}\right)$ denotes the generic point in $\mathbf{R}^{3}$.

Let $\Omega^{a}=\omega^{a} \times(0,1), \Omega^{b}=\omega^{b} \times(-1,0), T^{a}=\omega^{a} \times\{1\}, \Sigma^{a}=\partial \omega^{a} \times(0,1)$ and $\Sigma^{b}=\partial \omega^{b} \times(-1,0)$. The asymptotic behaviour of $\bar{U}^{\varepsilon}$ can be described by using a convenient rescaling (the reader is referred to Sect. 3.1 for details). This rescaling maps the space $Y^{\varepsilon}$ onto the space $\mathcal{Y}^{\varepsilon}$ defined by:

$$
\begin{aligned}
\mathcal{Y}^{\varepsilon}=\{u= & \left(u^{a}, u^{b}\right) \in\left(H^{1}\left(\Omega^{a}\right)\right)^{3} \times\left(H^{1}\left(\Omega^{b}\right)\right)^{3}, u^{a}=0 \text { on } T^{a}, u^{b}=0 \text { on } \Sigma^{b}, \\
& \left.u_{\alpha}^{a}\left(x^{\prime}, 0\right)=\varepsilon r^{\varepsilon} u_{\alpha}^{b}\left(r^{\varepsilon} x^{\prime}, 0\right) \text { and } u_{3}^{a}\left(x^{\prime}, 0\right)=u_{3}^{b}\left(r^{\varepsilon} x^{\prime}, 0\right), \text { for a.e. } x^{\prime} \in \omega^{a}\right\} .
\end{aligned}
$$

In particular, we denote by $\bar{u}^{\varepsilon}=\left(\bar{u}^{a \varepsilon}, \bar{u}^{b \varepsilon}\right)$ the rescaling of the solution $\bar{U}^{\varepsilon}$ of problem (1.2). We set

$$
e^{a \varepsilon}\left(u^{a}\right)=\left(\begin{array}{cc}
\frac{1}{\left(r^{\varepsilon}\right)^{2}} e_{\alpha \beta}\left(u^{a}\right) & \frac{1}{r^{\varepsilon}} e_{\alpha 3}\left(u^{a}\right) \\
\frac{1}{r^{\varepsilon}} e_{3 \alpha}\left(u^{a}\right) & e_{33}\left(u^{a}\right)
\end{array}\right), e^{b \varepsilon}\left(u^{b}\right)=\left(\begin{array}{cc}
e_{\alpha \beta}\left(u^{b}\right) & \frac{1}{\varepsilon} e_{\alpha 3}\left(u^{b}\right) \\
\frac{1}{\varepsilon} e_{3 \alpha}\left(u^{b}\right) & \frac{1}{\varepsilon^{2}} e_{33}\left(u^{b}\right)
\end{array}\right) .
$$

Then $\bar{u}^{\varepsilon}$ is the unique solution of the following problem:

$$
\left\{\begin{array}{l}
\bar{u}^{\varepsilon} \in \mathcal{Y}^{\varepsilon} \text { and } \forall u \in \mathcal{Y}^{\varepsilon}, \\
\int_{\Omega^{a}}\left[A^{a} e^{a \varepsilon}\left(\bar{u}^{a \varepsilon}\right), e^{a \varepsilon}\left(u^{a}\right)\right] \mathrm{d} x+q^{\varepsilon} \int_{\Omega^{b}}\left[A^{b} e^{b \varepsilon}\left(\bar{u}^{b \varepsilon}\right), e^{b \varepsilon}\left(u^{b}\right)\right] \mathrm{d} x \\
=\int_{\Omega^{a}} f^{a \varepsilon} \cdot u^{a} \mathrm{~d} x+\int_{\Omega^{b}} f^{b \varepsilon} \cdot u^{b} \mathrm{~d} x+\int_{\Omega^{a}}\left[g^{a \varepsilon}, e^{a \varepsilon}\left(u^{a}\right)\right] \mathrm{d} x+\int_{\Omega^{b}}\left[g^{b \varepsilon}, e^{b \varepsilon}\left(u^{b}\right)\right] \mathrm{d} x \\
+\int_{\Sigma^{a}} h^{a \varepsilon} \cdot u^{a} \mathrm{~d} \sigma+\int_{\omega^{b}}\left(h_{+}^{b \varepsilon} \cdot u_{\mid x_{3}=0}^{b}+h_{-}^{b \varepsilon} \cdot u_{\mid x_{3}=-1}^{b}\right) \mathrm{d} x^{\prime}
\end{array}\right.
$$

where $q^{\varepsilon}$ is defined by:

$$
q^{\varepsilon}=k^{\varepsilon} \frac{\varepsilon^{3}}{\left(r^{\varepsilon}\right)^{2}}
$$

and where the source terms are suitable transforms of $\left(F^{\varepsilon}, G^{\varepsilon}, H^{\varepsilon}\right)$ (see Sect. 3.1). 


\subsection{The setting of the limit problem}

For the definition of the limit problem, we introduce the following functional spaces:

$$
\begin{aligned}
& \mathcal{U}^{a}=\left\{u^{a} \in\left(H_{0}^{2}(0,1)\right)^{2} \times H^{1}\left(\Omega^{a}\right), \exists \zeta^{a} \in H^{1}(0,1), \zeta^{a}(1)=0, u_{3}^{a}=\zeta^{a}-x_{1} \frac{\mathrm{d} u_{1}^{a}}{\mathrm{~d} x_{3}}-x_{2} \frac{\mathrm{d} u_{2}^{a}}{\mathrm{~d} x_{3}}\right\} \\
& \mathcal{V}^{a}=\left\{v^{a} \in\left(H^{1}\left(\Omega^{a}\right)\right)^{2} \times L^{2}\left(0,1 ; H^{1}\left(\omega^{a}\right)\right), \exists c \in H_{0}^{1}(0,1), v_{1}^{a}=-c x_{2}, v_{2}^{a}=c x_{1},\right. \\
& \left.\mathcal{W}_{\omega^{a}} v_{3}^{a}\left(x^{\prime}, x_{3}\right) \mathrm{d} x^{\prime}=0, \text { for a.e. } x_{3} \in(0,1)\right\}, \\
& \mathcal{U}^{b}=\left\{w^{a} \in\left(L^{2}\left(0,1 ; H^{1}\left(\omega^{a}\right)\right)\right)^{2} \times\{0\}, \int_{\omega^{a}} w_{\alpha}^{a} \mathrm{~d} x^{\prime}=\int_{\omega^{a}}\left(x_{1} w_{2}^{a}-x_{2} w_{1}^{a}\right) \mathrm{d} x^{\prime}=0, \text { for a.e. } x_{3} \in(0,1)\right\}, \\
& \mathcal{V}^{b}=\left\{\Omega^{b} \in\left(L^{2}\left(\omega^{b} ; H^{1}(-1,0)\right)\right)^{2} \times\{0\}, \int_{-1}^{2}\left(\omega^{b}\right), \exists \zeta_{\alpha}^{b} \in H_{0}^{1}\left(\omega^{b}\right), u_{\alpha}^{b}=\zeta_{\alpha}^{b}-x_{3} \frac{\partial u_{3}^{b}}{\partial x_{\alpha}}\right\}, \\
& \mathcal{W}^{b}=\left\{w^{b} \in(\{0\})^{2} \times L^{2}\left(\omega^{b} ; H^{1}(-1,0)\right), \int_{-1}^{0} w_{3}^{b}\left(x^{\prime}, x_{3}\right) \mathrm{d} x_{3}=0, \text { for a.e. } x^{\prime} \in \omega^{b}\right\} \\
& \mathcal{Z}^{a}=\mathcal{U}^{a} \times \mathcal{V}^{a} \times \mathcal{W}^{a}, \mathcal{Z}^{b}=\mathcal{U}^{b} \times \mathcal{V}^{b} \times \mathcal{W}^{b} .
\end{aligned}
$$

Without loss of generality, we assume that $q^{\varepsilon}$ defined by (2.4) satisfies:

$$
q^{\varepsilon} \rightarrow q, \text { with } \quad 0 \leq q \leq+\infty
$$

According to the value of $q$, the functional space for the limit problem is the following one:

$$
\begin{array}{rlr}
\mathcal{Z} & =\left\{z=\left(z^{a}, z^{b}\right)=\left(\left(u^{a}, v^{a}, w^{a}\right),\left(u^{b}, v^{b}, w^{b}\right)\right) \in \mathcal{Z}^{a} \times \mathcal{Z}^{b}, u_{3}^{a}\left(x^{\prime}, 0\right)=u_{3}^{b}(0), \text { for a.e. } x^{\prime} \in \omega^{a}\right\}, \text { if } 0<q<+\infty \\
\mathcal{Z}_{\infty} & =\left\{z^{a}=\left(u^{a}, v^{a}, w^{a}\right) \in \mathcal{Z}^{a}, u_{3}^{a}\left(x^{\prime}, 0\right)=0, \text { for a.e. } x^{\prime} \in \omega^{a}\right\}, & \text { if } q=+\infty, \\
\mathcal{Z}_{0} & =\left\{z^{b}=\left(u^{b}, v^{b}, w^{b}\right) \in \mathcal{Z}^{b}, u_{3}^{b}(0)=0\right\}, & \text { if } q=0 .
\end{array}
$$

Let us observe that $\mathcal{U}^{a}$ (resp. $\mathcal{U}^{b}$ ) is a Bernoulli-Navier (resp. Kirchhoff-Love) space of displacements. Less classical spaces are $\mathcal{V}^{a}, \mathcal{W}^{a}, \mathcal{V}^{b}, \mathcal{W}^{b}$, which are introduced in a way similar to $[27,28]$ (see also App., Sect. 8.1). As for the boundary conditions, some of them are due to the clamping. These are more or less standard ones:

$$
u_{\alpha}^{a}(1)=\frac{\mathrm{d} u_{\alpha}^{a}}{\mathrm{~d} x_{3}}(1)=c(1)=0, u_{3}^{b}=0 \text { and } \frac{\partial u_{3}^{b}}{\mathrm{~d} \nu}=0 \text { on } \partial \omega^{b} .
$$

In contrast with the other requirements, the six conditions:

$$
u_{\alpha}^{a}(0)=\frac{\mathrm{d} u_{\alpha}^{a}}{\mathrm{~d} x_{3}}(0)=c(0)=0, u_{3}^{a}\left(x^{\prime}, 0\right)=u_{3}^{b}(0)\left(\operatorname{respectively} u_{3}^{a}\left(x^{\prime}, 0\right)=0 \text { or } u_{3}^{b}(0)=0\right),
$$

which appear in the definition of the above spaces $\mathcal{U}^{a}, \mathcal{V}^{a}$ and $\mathcal{Z}$ (respectively $\mathcal{Z}_{\infty}$ or $\mathcal{Z}_{0}$ ), are specific to the junction between the beam and the plate. Note also that, in view of the definition of $\mathcal{U}^{a}$, the condition $u_{3}^{a}\left(x^{\prime}, 0\right)=u_{3}^{b}(0)$ (respectively $u_{3}^{a}\left(x^{\prime}, 0\right)=0$ ) reduces to $\zeta^{a}(0)=u_{3}^{b}(0)$ (respectively $\left.\zeta^{a}(0)=0\right)$. 
We finally introduce, for $z^{a}=\left(u^{a}, v^{a}, w^{a}\right)$ in $\mathcal{Z}^{a}$ and $z^{b}=\left(u^{b}, v^{b}, w^{b}\right)$ in $\mathcal{Z}^{b}$ :

$$
e^{a}\left(z^{a}\right)=\left(\begin{array}{cc}
e_{\alpha \beta}\left(w^{a}\right) & e_{\alpha 3}\left(v^{a}\right) \\
e_{3 \alpha}\left(v^{a}\right) & e_{33}\left(u^{a}\right)
\end{array}\right), e^{b}\left(z^{b}\right)=\left(\begin{array}{cc}
e_{\alpha \beta}\left(u^{b}\right) & e_{\alpha 3}\left(v^{b}\right) \\
e_{3 \alpha}\left(v^{b}\right) & e_{33}\left(w^{b}\right)
\end{array}\right) .
$$

\subsection{The main result}

We describe the limit behaviour of problem (2.3), as $\varepsilon$ tends to zero. In the sequel, we assume that

$$
\begin{gathered}
f^{a \varepsilon} \rightarrow f^{a} \text { weakly in }\left(L^{2}\left(\Omega^{a}\right)\right)^{3}, \\
f^{b \varepsilon} \rightarrow f^{b} \text { weakly in }\left(L^{2}\left(\Omega^{b}\right)\right)^{3}, \\
g^{a \varepsilon} \rightarrow g^{a} \text { weakly in }\left(L^{2}\left(\Omega^{a}\right)\right)^{3 \times 3}, \\
g^{b \varepsilon} \rightarrow g^{b} \text { weakly in }\left(L^{2}\left(\Omega^{b}\right)\right)^{3 \times 3}, \\
h^{a \varepsilon} \rightarrow h^{a} \text { weakly in }\left(L^{2}\left(\Sigma^{a}\right)\right)^{3}, \\
h_{+}^{b \varepsilon} \rightarrow h_{+}^{b} \text { and } h_{-}^{b \varepsilon} \rightarrow h_{-}^{b} \text { weakly in }\left(L^{2}\left(\omega^{b}\right)\right)^{3},
\end{gathered}
$$

which is not restrictive, as proved in Remark 4 below.

Our main result is the following one:

Theorem 1. Assume that $\frac{r^{\varepsilon}}{\varepsilon^{2}}$ tends to $+\infty$ and that $(2.5),(2.7)$ to $(2.12)$ hold true. Then, with the notation $e^{a \varepsilon}, e^{b \varepsilon}$ defined in (2.2) and $e^{a}, e^{b}$ defined in (2.6), one has:

(i) If $0<q<+\infty$, there exists $\bar{z}=\left(\bar{z}^{a}, \bar{z}^{b}\right)=\left(\left(\bar{u}^{a}, \bar{v}^{a}, \bar{w}^{a}\right),\left(\bar{u}^{b}, \bar{v}^{b}, \bar{w}^{b}\right)\right) \in \mathcal{Z}$ such that:

$$
\begin{gathered}
\left(\bar{u}^{a \varepsilon}, \bar{u}^{b \varepsilon}\right) \rightarrow\left(\bar{u}^{a}, \bar{u}^{b}\right) \text { weakly in }\left(H^{1}\left(\Omega^{a}\right)\right)^{3} \times\left(H^{1}\left(\Omega^{b}\right)\right)^{3}, \\
\left(e^{a \varepsilon}\left(\bar{u}^{a \varepsilon}\right), e^{b \varepsilon}\left(\bar{u}^{b \varepsilon}\right)\right) \rightarrow\left(e^{a}\left(\bar{z}^{a}\right), e^{b}\left(\bar{z}^{b}\right)\right) \text { weakly in }\left(L^{2}\left(\Omega^{a}\right)\right)^{3 \times 3} \times\left(L^{2}\left(\Omega^{b}\right)\right)^{3 \times 3},
\end{gathered}
$$

and $\bar{z}$ is the unique solution of the following problem:

$$
\left\{\begin{array}{l}
\bar{z} \in \mathcal{Z} \text { and } \forall z \in \mathcal{Z} \\
\int_{\Omega^{a}}\left[A^{a} e^{a}\left(\bar{z}^{a}\right), e^{a}\left(z^{a}\right)\right] \mathrm{d} x+q \int_{\Omega^{b}}\left[A^{b} e^{b}\left(\bar{z}^{b}\right), e^{b}\left(z^{b}\right)\right] \mathrm{d} x \\
=\int_{\Omega^{a}} f^{a} \cdot u^{a} \mathrm{~d} x+\int_{\Omega^{b}} f^{b} \cdot u^{b} \mathrm{~d} x+\int_{\Omega^{a}}\left[g^{a}, e^{a}\left(z^{a}\right)\right] \mathrm{d} x+\int_{\Omega^{b}}\left[g^{b}, e^{b}\left(z^{b}\right)\right] \mathrm{d} x \\
+\int_{\Sigma^{a}} h^{a} \cdot u^{a} \mathrm{~d} \sigma+\int_{\omega^{b}}\left(h_{+}^{b} \cdot u_{\mid x_{3}=0}^{b}+h_{-}^{b} \cdot u_{\mid x_{3}=-1}^{b}\right) \mathrm{d} x^{\prime}
\end{array}\right.
$$

Moreover, if the convergences in (2.9), (2.10) are strong, then the convergences in (2.13) and (2.14) are strong. 
(ii) If $q=+\infty$, there exists $\bar{z}^{a}=\left(\bar{u}^{a}, \bar{v}^{a}, \bar{w}^{a}\right) \in \mathcal{Z}_{\infty}$ such that:

$$
\begin{gathered}
\bar{u}^{a \varepsilon} \rightarrow \bar{u}^{a} \text { weakly in }\left(H^{1}\left(\Omega^{a}\right)\right)^{3}, \bar{u}^{b \varepsilon} \rightarrow 0 \text { strongly in }\left(H^{1}\left(\Omega^{b}\right)\right)^{3}, \\
e^{a \varepsilon}\left(\bar{u}^{a \varepsilon}\right) \rightarrow e^{a}\left(\bar{z}^{a}\right) \text { weakly in }\left(L^{2}\left(\Omega^{a}\right)\right)^{3 \times 3}, \quad e^{b \varepsilon}\left(\bar{u}^{b \varepsilon}\right) \rightarrow 0 \text { strongly in }\left(L^{2}\left(\Omega^{b}\right)\right)^{3 \times 3},
\end{gathered}
$$

and $\bar{z}^{a}$ is the unique solution of the following problem:

$$
\left\{\begin{array}{l}
\bar{z}^{a} \in \mathcal{Z}_{\infty} \text { and } \forall z^{a} \in \mathcal{Z}_{\infty} \\
\int_{\Omega^{a}}\left[A^{a} e^{a}\left(\bar{z}^{a}\right), e^{a}\left(z^{a}\right)\right] \mathrm{d} x=\int_{\Omega^{a}} f^{a} \cdot u^{a} \mathrm{~d} x+\int_{\Omega^{a}}\left[g^{a}, e^{a}\left(z^{a}\right)\right] \mathrm{d} x+\int_{\Sigma^{a}} h^{a} \cdot u^{a} \mathrm{~d} \sigma
\end{array}\right.
$$

Moreover, if the convergence in (2.9) is strong, then:

$$
\begin{gathered}
\bar{u}^{a \varepsilon} \rightarrow \bar{u}^{a} \text { strongly in }\left(H^{1}\left(\Omega^{a}\right)\right)^{3}, \\
e^{a \varepsilon}\left(\bar{u}^{a \varepsilon}\right) \rightarrow e^{a}\left(\bar{z}^{a}\right) \text { strongly in }\left(L^{2}\left(\Omega^{a}\right)\right)^{3 \times 3}, \sqrt{q^{\varepsilon}} e^{b \varepsilon}\left(\bar{u}^{b \varepsilon}\right) \rightarrow 0 \text { strongly in }\left(L^{2}\left(\Omega^{b}\right)\right)^{3 \times 3} .
\end{gathered}
$$

(iii) If $q=0$, there exists $\bar{z}^{b}=\left(\bar{u}^{b}, \bar{v}^{b}, \bar{w}^{b}\right) \in \mathcal{Z}_{0}$ such that:

$$
\begin{gathered}
q^{\varepsilon} \bar{u}^{a \varepsilon} \rightarrow 0 \text { strongly in }\left(H^{1}\left(\Omega^{a}\right)\right)^{3}, q^{\varepsilon} \bar{u}^{b \varepsilon} \rightarrow \bar{u}^{b} \text { weakly in }\left(H^{1}\left(\Omega^{b}\right)\right)^{3}, \\
q^{\varepsilon} e^{a \varepsilon}\left(\bar{u}^{a \varepsilon}\right) \rightarrow 0 \text { strongly in }\left(L^{2}\left(\Omega^{a}\right)\right)^{3 \times 3}, q^{\varepsilon} e^{b \varepsilon}\left(\bar{u}^{b \varepsilon}\right) \rightarrow e^{b}\left(\bar{z}^{b}\right) \text { weakly in }\left(L^{2}\left(\Omega^{b}\right)\right)^{3 \times 3},
\end{gathered}
$$

and $\bar{z}^{b}$ is the unique solution of the following problem:

$$
\left\{\begin{array}{l}
\bar{z}^{b} \in \mathcal{Z}_{0} \text { and } \forall z^{b} \in \mathcal{Z}_{0}, \\
\int_{\Omega^{b}}\left[A^{b} e^{b}\left(\bar{z}^{b}\right), e^{b}\left(z^{b}\right)\right] \mathrm{d} x=\int_{\Omega^{b}} f^{b} \cdot u^{b} \mathrm{~d} x+\int_{\Omega^{b}}\left[g^{b}, e^{b}\left(z^{b}\right)\right] \mathrm{d} x+\int_{\omega^{b}}\left(h_{+}^{b} \cdot u_{\mid x_{3}=0}^{b}+h_{-}^{b} \cdot u_{\mid x_{3}=-1}^{b}\right) \mathrm{d} x^{\prime} .
\end{array}\right.
$$

Moreover, if the convergence in (2.10) is strong, then:

$$
\begin{gathered}
q^{\varepsilon} \bar{u}^{b \varepsilon} \rightarrow \bar{u}^{b} \text { strongly in }\left(H^{1}\left(\Omega^{b}\right)\right)^{3}, \\
\sqrt{q^{\varepsilon}} e^{a \varepsilon}\left(\bar{u}^{a \varepsilon}\right) \rightarrow 0 \text { strongly in }\left(L^{2}\left(\Omega^{b}\right)\right)^{3 \times 3}, q^{\varepsilon} e^{b \varepsilon}\left(\bar{u}^{b \varepsilon}\right) \rightarrow e^{b}\left(\bar{z}^{b}\right) \text { strongly in }\left(L^{2}\left(\Omega^{b}\right)\right)^{3 \times 3} .
\end{gathered}
$$

Remark 1. The condition that $\frac{r^{\varepsilon}}{\varepsilon^{2}}$ tends to $+\infty$ is only used to prove that $\bar{u}_{3}^{a}\left(x^{\prime}, 0\right)=\bar{u}_{3}^{b}(0)$ and $\bar{c}(0)=0$ (via a convenient Sobolev embedding theorem, as regards the second equality). We do not know if it is just a technical condition or not.

Remark 2. In the Appendix (Sect. 8.1) we prove that the functions $\bar{v}^{a}$ and $\bar{w}^{a}$ (resp. $\bar{v}^{b}$ and $\bar{w}^{b}$ ) which appear in the limit problem are the limits of suitable expressions of $\bar{u}^{a \varepsilon}\left(\right.$ resp. $\left.\bar{u}^{b \varepsilon}\right)$. 


\subsection{Back to the problem in the thin multidomain}

As far as the asymptotic behaviour of the "energy" of the solution of problem (1.2) in the thin multidomain is concerned, we define the following renormalized energy by:

$$
\mathcal{E}^{\varepsilon}=\left(\frac{\lambda^{\varepsilon}}{r^{\varepsilon}}\right)^{2} \int_{\Omega^{\varepsilon}}\left[A^{\varepsilon} e\left(\bar{U}^{\varepsilon}\right), e\left(\bar{U}^{\varepsilon}\right)\right] \mathrm{d} x
$$

where $\lambda^{\varepsilon}$ can be made explicit in terms of $\varepsilon, r^{\varepsilon}, F^{\varepsilon}, G^{\varepsilon}, H^{\varepsilon}$ (see (3.2) in Sect. 3.1); we also have:

$$
\mathcal{E}^{\varepsilon}=\int_{\Omega^{a}}\left[A^{a} e^{a \varepsilon}\left(\bar{u}^{a \varepsilon}\right), e^{a \varepsilon}\left(\bar{u}^{a \varepsilon}\right)\right] \mathrm{d} x+q^{\varepsilon} \int_{\Omega^{b}}\left[A^{b} e^{b \varepsilon}\left(\bar{u}^{b \varepsilon}\right), e^{b \varepsilon}\left(\bar{u}^{b \varepsilon}\right)\right] \mathrm{d} x,
$$

and from Theorem 1 we deduce the following corollary:

Corollary 1. Assume that $\frac{r^{\varepsilon}}{\varepsilon^{2}}$ tends to $+\infty$ and that (2.5), (2.7) to (2.12) hold true.

(i) If $0<q<+\infty$ and if the convergences in (2.9), (2.10) are strong, then:

$$
\mathcal{E}^{\mathcal{E}} \rightarrow \mathcal{E}=\int_{\Omega^{a}}\left[A^{a} e^{a}\left(\bar{z}^{a}\right), e^{a}\left(\bar{z}^{a}\right)\right] \mathrm{d} x+q \int_{\Omega^{b}}\left[A^{b} e^{b}\left(\bar{z}^{b}\right), e^{b}\left(\bar{z}^{b}\right)\right] \mathrm{d} x .
$$

(ii) If $q=+\infty$ and if the convergence in (2.9) is strong, then:

$$
\mathcal{E}^{\varepsilon} \rightarrow \mathcal{E}_{\infty}=\int_{\Omega^{a}}\left[A^{a} e^{a}\left(\bar{z}^{a}\right), e^{a}\left(\bar{z}^{a}\right)\right] \mathrm{d} x .
$$

(iii) If $q=0$ and if the convergence in (2.10) is strong, then:

$$
q^{\varepsilon} \mathcal{E}^{\varepsilon} \rightarrow \mathcal{E}_{0}=\int_{\Omega^{b}}\left[A^{b} e^{b}\left(\bar{z}^{b}\right), e^{b}\left(\bar{z}^{b}\right)\right] \mathrm{d} x .
$$

Actually, the proof of Corollary 1 is part of proof of Theorem 1, since the strong convergences of $\bar{u}^{a \varepsilon}$ to $\bar{u}^{a}$ (resp. $\bar{u}^{b \varepsilon}$ to $\bar{u}^{b}$ ) and $e^{a \varepsilon}\left(\bar{u}^{a \varepsilon}\right)$ to $e^{a}\left(\bar{z}^{a}\right)$ (resp. $e^{b \varepsilon}\left(\bar{u}^{b \varepsilon}\right)$ to $\left.e^{b}\left(\bar{z}^{b}\right)\right)$ follow from the convergence of the energy $\mathcal{E}^{\varepsilon}$. The following interpretation is a direct consequence of the strong convergences of $e^{a \varepsilon}\left(\bar{u}^{a \varepsilon}\right)$ and $e^{b \varepsilon}\left(\bar{u}^{b \varepsilon}\right)$.

Interpretation. For example, let us consider the particular case of problem (1.2), for which $G^{\varepsilon}=0, H^{\varepsilon}=0$, $k^{\varepsilon}=1$ and $A^{a}=A^{b}=A$ :

$$
\left\{\begin{array}{l}
\bar{U}^{\varepsilon} \in Y^{\varepsilon} \text { and } \forall U \in Y^{\varepsilon}, \\
\int_{\Omega^{\varepsilon}}\left[A e\left(\bar{U}^{\varepsilon}\right), e(U)\right] \mathrm{d} x=\int_{\Omega^{\varepsilon}} F^{\varepsilon} \cdot U \mathrm{~d} x,
\end{array}\right.
$$

and let us assume that $r^{\varepsilon}=\varepsilon^{3 / 2}$ and that:

$$
\frac{1}{\varepsilon^{9}} \sum_{\alpha}\left\|F_{\alpha}^{\varepsilon}\right\|_{L^{2}\left(\Omega^{a \varepsilon}\right)}^{2}+\frac{1}{\varepsilon^{6}} \sum_{\alpha}\left\|F_{3}^{\varepsilon}\right\|_{L^{2}\left(\Omega^{a \varepsilon}\right)}^{2}+\frac{1}{\varepsilon^{6}} \sum_{\alpha}\left\|F_{\alpha}^{\varepsilon}\right\|_{L^{2}\left(\Omega^{b \varepsilon}\right)}^{2}+\frac{1}{\varepsilon^{8}} \sum_{\alpha}\left\|F_{3}^{\varepsilon}\right\|_{L^{2}\left(\Omega^{b \varepsilon}\right)}^{2}=1 .
$$


This last condition is not restrictive, since it is just a matter of normalization. One can observe that, in this case, the parameter $\lambda^{\varepsilon}$ introduced in (3.2), in Section 3.1, has value $\varepsilon^{-3 / 2}$. Defining the rescaled force and the rescaled solution by:

$$
\begin{array}{lll}
f_{\alpha}^{a \varepsilon}(x)=\frac{1}{\varepsilon^{3}} F_{\alpha}^{\varepsilon}\left(\varepsilon^{\frac{3}{2}} x^{\prime}, x_{3}\right), & f_{3}^{a \varepsilon}(x)=\frac{1}{\varepsilon^{\frac{3}{2}}} F_{3}^{\varepsilon}\left(\varepsilon^{\frac{3}{2}} x^{\prime}, x_{3}\right), & \text { for } x \in \Omega^{a}, \\
f_{\alpha}^{b \varepsilon}(x)=\frac{1}{\varepsilon^{\frac{5}{2}}} F_{\alpha}^{\varepsilon}\left(x^{\prime}, \varepsilon x_{3}\right), & f_{3}^{b \varepsilon}(x)=\frac{1}{\varepsilon^{\frac{7}{2}}} F_{3}^{\varepsilon}\left(x^{\prime}, \varepsilon x_{3}\right), & \text { for } x \in \Omega^{b}, \\
\bar{u}_{\alpha}^{a \varepsilon}(x)=\bar{U}_{\alpha}^{\varepsilon}\left(\varepsilon^{\frac{3}{2}} x^{\prime}, x_{3}\right), & \bar{u}_{3}^{a \varepsilon}(x)=\frac{1}{\varepsilon^{\frac{3}{2}}} \bar{U}_{3}^{\varepsilon}\left(\varepsilon^{\frac{3}{2}} x^{\prime}, x_{3}\right), & \text { for } x \in \Omega^{a}, \\
\bar{u}_{\alpha}^{b \varepsilon}(x)=\frac{1}{\varepsilon^{\frac{5}{2}}} \bar{U}_{\alpha}^{\varepsilon}\left(x^{\prime}, \varepsilon x_{3}\right), & \bar{u}_{3}^{b \varepsilon}(x)=\frac{1}{\varepsilon^{\frac{3}{2}}} \bar{U}_{3}^{\varepsilon}\left(x^{\prime}, \varepsilon x_{3}\right), & \text { for } x \in \Omega^{b},
\end{array}
$$

one can check that $\bar{u}^{\varepsilon}$ solves the rescaled problem:

$$
\left\{\begin{array}{l}
\bar{u}^{\varepsilon} \in \mathcal{Y}^{\varepsilon} \text { and } \forall u \in \mathcal{Y}^{\varepsilon}, \\
\int_{\Omega^{a}}\left[A e^{a \varepsilon}\left(\bar{u}^{a \varepsilon}\right), e^{a \varepsilon}\left(u^{a}\right)\right] \mathrm{d} x+\int_{\Omega^{b}}\left[A e^{b \varepsilon}\left(\bar{u}^{b \varepsilon}\right), e^{b \varepsilon}\left(u^{b}\right)\right] \mathrm{d} x=\int_{\Omega^{a}} f^{a \varepsilon} \cdot u^{a} \mathrm{~d} x+\int_{\Omega^{b}} f^{b \varepsilon} \cdot u^{b} \mathrm{~d} x .
\end{array}\right.
$$

Since, thanks to (2.27),

$$
\int_{\Omega^{a}}\left|f^{a \varepsilon}\right|^{2} \mathrm{~d} x+\int_{\Omega^{b}}\left|f^{b \varepsilon}\right|^{2} \mathrm{~d} x=1
$$

it is not restrictive to assume that, for some subsequence of $\varepsilon$, still denoted by $\varepsilon$, and for some $f^{a}$ in $L^{2}\left(\Omega^{a}\right)$ and $f^{b}$ in $L^{2}\left(\Omega^{b}\right)$ :

$$
f^{a \varepsilon} \rightarrow f^{a} \text { in } L^{2}\left(\Omega^{a}\right) \text { and } f^{b \varepsilon} \rightarrow f^{b} \text { in } L^{2}\left(\Omega^{b}\right) .
$$

Then, Theorem 1 asserts that:

$$
\begin{gathered}
\bar{u}^{a \varepsilon} \rightarrow \bar{u}^{a} \text { strongly in }\left(H^{1}\left(\Omega^{a}\right)\right)^{3} \text { and } \bar{u}^{b \varepsilon} \rightarrow \bar{u}^{b} \text { strongly in }\left(H^{1}\left(\Omega^{b}\right)\right)^{3}, \\
e^{a \varepsilon}\left(\bar{u}^{a \varepsilon}\right) \rightarrow \bar{e}^{a} \text { strongly in }\left(L^{2}\left(\Omega^{a}\right)\right)^{3 \times 3} \text { and } e^{b \varepsilon}\left(\bar{u}^{b \varepsilon}\right) \rightarrow \bar{e}^{b} \text { strongly in }\left(L^{2}\left(\Omega^{b}\right)\right)^{3 \times 3},
\end{gathered}
$$

where $\bar{e}^{a}=e^{a}\left(\bar{z}^{a}\right), \bar{e}^{b}=e^{b}\left(\bar{z}^{b}\right)$ and $\bar{z}=\left(\bar{z}^{a}, \bar{z}^{b}\right)$ is the unique solution of the rescaled limit problem:

$$
\left\{\begin{array}{l}
\bar{z} \in \mathcal{Z} \text { and } \forall z \in \mathcal{Z}, \\
\int_{\Omega^{a}}\left[A e^{a}\left(\bar{z}^{a}\right), e^{a}\left(z^{a}\right)\right] \mathrm{d} x+\int_{\Omega^{b}}\left[A e^{b}\left(\bar{z}^{b}\right), e^{b}\left(z^{b}\right)\right] \mathrm{d} x=\int_{\Omega^{a}} f^{a} \cdot u^{a} \mathrm{~d} x+\int_{\Omega^{b}} f^{b} \cdot u^{b} \mathrm{~d} x .
\end{array}\right.
$$

Coming back to the initial domain, we define $\bar{E}^{a \varepsilon}$ and $\bar{E}^{b \varepsilon}$ by:

$$
\bar{E}^{a \varepsilon}=\varepsilon^{\frac{3}{2}} \bar{e}^{a}\left(\frac{x^{\prime}}{r^{\varepsilon}}, x_{3}\right), \text { for } x \in \Omega^{a \varepsilon}, \quad \bar{E}^{b \varepsilon}=\varepsilon^{\frac{5}{2}} \bar{e}^{b}\left(x^{\prime}, \frac{x_{3}}{\varepsilon}\right), \text { for } x \in \Omega^{b \varepsilon},
$$


and we define the relative errors $\Delta^{a \varepsilon}, \Delta^{b \varepsilon}$ and $\Delta^{\varepsilon}$ by:

$$
\begin{gathered}
\Delta^{a \varepsilon}=\frac{\int_{\Omega^{a \varepsilon}}\left|e\left(\bar{U}^{\varepsilon}\right)-\bar{E}^{a \varepsilon}\right|^{2} \mathrm{~d} x}{\int_{\Omega^{a \varepsilon}}\left|\bar{E}^{a \varepsilon}\right|^{2} \mathrm{~d} x}, \quad \Delta^{b \varepsilon}=\frac{\int_{\Omega^{b \varepsilon}}\left|e\left(\bar{U}^{\varepsilon}\right)-\bar{E}^{b \varepsilon}\right|^{2} \mathrm{~d} x}{\int_{\Omega^{b \varepsilon}}\left|\bar{E}^{b \varepsilon}\right|^{2} \mathrm{~d} x}, \\
\Delta^{\varepsilon}=\frac{\int_{\Omega^{a \varepsilon}}\left|e\left(\bar{U}^{\varepsilon}\right)-\bar{E}^{a \varepsilon}\right|^{2} \mathrm{~d} x+\int_{\Omega^{b \varepsilon}}\left|e\left(\bar{U}^{\varepsilon}\right)-\bar{E}^{b \varepsilon}\right|^{2} \mathrm{~d} x}{\int_{\Omega^{a \varepsilon}}\left|\bar{E}^{a \varepsilon}\right|^{2} \mathrm{~d} x+\int_{\Omega^{b \varepsilon}}\left|\bar{E}^{b \varepsilon}\right|^{2} \mathrm{~d} x} .
\end{gathered}
$$

Assuming that $\bar{e}^{a} \neq 0$ and $\bar{e}^{b} \neq 0$, an easy computation gives that:

$$
\Delta^{a \varepsilon}=\frac{\int_{\Omega^{a}}\left|e^{a \varepsilon}\left(\bar{u}^{a \varepsilon}\right)-\bar{e}^{a}\right|^{2} \mathrm{~d} x}{\int_{\Omega^{a}}\left|\bar{e}^{a}\right|^{2} \mathrm{~d} x}, \quad \Delta^{b \varepsilon}=\frac{\int_{\Omega^{b}}\left|e^{b \varepsilon}\left(\bar{u}^{b \varepsilon}\right)-\bar{e}^{b}\right|^{2} \mathrm{~d} x}{\int_{\Omega^{b}}\left|\bar{e}^{b}\right|^{2} \mathrm{~d} x} .
$$

Hence the strong convergences in (2.29) imply that $\Delta^{a \varepsilon}, \Delta^{b \varepsilon}$, and then $\Delta^{\varepsilon}$, tend to zero with $\varepsilon$. These convergences of the relative errors mean that the deformation of the original displacement is well described by $\bar{E}^{a \varepsilon}$ and $\bar{E}^{b \varepsilon}$ :

$$
e\left(\bar{U}^{\varepsilon}\right) \simeq \bar{E}^{a \varepsilon} \text { in } \Omega^{a \varepsilon}, \quad e\left(\bar{U}^{\varepsilon}\right) \simeq \bar{E}^{b \varepsilon} \text { in } \Omega^{b \varepsilon} .
$$

In the same spirit, from the solution $\bar{z}=\left(\bar{z}^{a}, \bar{z}^{b}\right)=\left(\left(\bar{u}^{a}, \bar{v}^{a}, \bar{w}^{a}\right),\left(\bar{u}^{b}, \bar{v}^{b}, \bar{w}^{b}\right)\right)$ of problem (2.30), we are going to define $\hat{U}^{a \varepsilon}$ and $\hat{U}^{b \varepsilon}$, which are good approximates of the restrictions of $\bar{U}^{\varepsilon}$ to $\Omega^{a \varepsilon}$ and $\Omega^{b \varepsilon}$, respectively. Actually, let us set:

$$
\begin{gathered}
\hat{u}^{a \varepsilon}=\bar{u}^{a}+r^{\varepsilon} \bar{v}^{a}+\left(r^{\varepsilon}\right)^{2} \bar{w}^{a}=\bar{u}^{a}+\varepsilon^{\frac{3}{2}} \bar{v}^{a}+\varepsilon^{3} \bar{w}^{a}, \\
\hat{u}^{b \varepsilon}=\bar{u}^{b}+\varepsilon \bar{v}^{b}+\varepsilon^{2} \bar{w}^{b}, \\
\hat{U}_{\alpha}^{a \varepsilon}(x)=\hat{u}_{\alpha}^{a \varepsilon}\left(\frac{x^{\prime}}{\varepsilon^{\frac{3}{2}}}, x_{3}\right), \quad \hat{U}_{3}^{a \varepsilon}(x)=\varepsilon^{\frac{3}{2}} \hat{u}_{3}^{a \varepsilon}\left(\frac{x^{\prime}}{\varepsilon^{\frac{3}{2}}}, x_{3}\right), \quad \text { for } x \in \Omega^{a \varepsilon}, \\
\hat{U}_{\alpha}^{b \varepsilon}(x)=\varepsilon^{\frac{5}{2}} \hat{u}_{\alpha}^{b \varepsilon}\left(x^{\prime}, \frac{x_{3}}{\varepsilon}\right), \quad \hat{U}_{3}^{b \varepsilon}(x)=\varepsilon^{\frac{3}{2}} \hat{u}_{3}^{b \varepsilon}\left(x^{\prime}, \frac{x_{3}}{\varepsilon}\right), \quad \text { for } x \in \Omega^{b \varepsilon} .
\end{gathered}
$$

Assuming that $\left(\bar{v}^{a}, \bar{w}^{a}\right),\left(\bar{v}^{b}, \bar{w}^{b}\right)$ have $H^{1}$ regularity, and since:

$$
e^{a \varepsilon}\left(\hat{u}^{a \varepsilon}\right)=\bar{e}^{a}+\varepsilon^{\frac{3}{2}}\left(\begin{array}{cc}
0 & e_{\alpha 3}\left(\bar{w}^{a}\right) \\
e_{3 \beta}\left(\bar{w}^{a}\right) & e_{33}\left(\bar{v}^{a}\right)
\end{array}\right), \quad e^{b \varepsilon}\left(\hat{u}^{b \varepsilon}\right)=\bar{e}^{b}+\varepsilon\left(\begin{array}{cc}
e_{\alpha \beta}\left(\bar{v}^{b}\right) & e_{\alpha 3}\left(\bar{w}^{b}\right) \\
e_{3 \beta}\left(\bar{w}^{b}\right) & 0
\end{array}\right),
$$

it is clear that, as $\varepsilon$ tends to zero, $e^{a \varepsilon}\left(\hat{u}^{a \varepsilon}\right)$ tends to $\bar{e}^{a}$ strongly in $\left(L^{2}\left(\Omega^{a}\right)\right)^{3 \times 3}$ and that $e^{b \varepsilon}\left(\hat{u}^{b \varepsilon}\right)$ tends to $\bar{e}^{b}$ strongly in $\left(L^{2}\left(\Omega^{b}\right)\right)^{3 \times 3}$, and then, from (2.29), that:

$$
\int_{\Omega^{a}}\left|e^{a \varepsilon}\left(\bar{u}^{a \varepsilon}-\hat{u}^{a \varepsilon}\right)\right|^{2} \mathrm{~d} x+\int_{\Omega^{b}}\left|e^{b \varepsilon}\left(\bar{u}^{b \varepsilon}-\hat{u}^{b \varepsilon}\right)\right|^{2} \mathrm{~d} x \rightarrow 0 .
$$


As above, if $\bar{e}^{a} \neq 0$ and $\bar{e}^{b} \neq 0$, we get that:

At least formally, this means that:

$$
\begin{aligned}
\hat{\Delta}^{a \varepsilon} & =\frac{\int_{\Omega^{a \varepsilon}}\left|e\left(\bar{U}^{\varepsilon}-\hat{U}^{a \varepsilon}\right)\right|^{2} \mathrm{~d} x}{\int_{\Omega^{a \varepsilon}}\left|e\left(\hat{U}^{a \varepsilon}\right)\right|^{2} \mathrm{~d} x}=\frac{\int_{\Omega^{a}}\left|e^{a \varepsilon}\left(\bar{u}^{a \varepsilon}-\hat{u}^{a \varepsilon}\right)\right|^{2} \mathrm{~d} x}{\int_{\Omega^{a}}\left|e^{a \varepsilon}\left(\hat{u}^{a \varepsilon}\right)\right|^{2} \mathrm{~d} x} \rightarrow 0, \\
\hat{\Delta}^{b \varepsilon} & =\frac{\int_{\Omega^{b \varepsilon}}\left|e\left(\bar{U}^{\varepsilon}-\hat{U}^{b \varepsilon}\right)\right|^{2} \mathrm{~d} x}{\int_{\Omega^{b \varepsilon}}\left|e\left(\hat{U}^{b \varepsilon}\right)\right|^{2} \mathrm{~d} x}=\frac{\int_{\Omega^{b}}\left|e^{b \varepsilon}\left(\bar{u}^{b \varepsilon}-\hat{u}^{b \varepsilon}\right)\right|^{2} \mathrm{~d} x}{\int_{\Omega^{b}}\left|e^{b \varepsilon}\left(\hat{u}^{b \varepsilon}\right)\right|^{2} \mathrm{~d} x} \rightarrow 0, \\
\hat{\Delta}^{\varepsilon} & =\frac{\int_{\Omega^{a \varepsilon}}\left|e\left(\bar{U}^{\varepsilon}-\hat{U}^{a \varepsilon}\right)\right|^{2} \mathrm{~d} x+\int_{\Omega^{b \varepsilon}}\left|e\left(\bar{U}^{\varepsilon}-\hat{U}^{b \varepsilon}\right)\right|^{2} \mathrm{~d} x}{\int_{\Omega^{a \varepsilon}}\left|e\left(\hat{U}^{a \varepsilon}\right)\right|^{2} \mathrm{~d} x+\int_{\Omega^{b \varepsilon}}\left|e\left(\hat{U}^{b \varepsilon}\right)\right|^{2} \mathrm{~d} x} \rightarrow 0 .
\end{aligned}
$$

$$
\bar{U}^{\varepsilon} \simeq \hat{U}^{a \varepsilon} \text { in } \Omega^{a \varepsilon}, \quad \bar{U}^{\varepsilon} \simeq \hat{U}^{b \varepsilon} \text { in } \Omega^{b \varepsilon} .
$$

Let us prove that, from the equivalence (2.31), one can recover heuristically the conditions at the junction. As a matter of fact, suppose we just know that:

$$
\begin{gathered}
\bar{u}_{\alpha}^{a}=\bar{u}_{\alpha}^{a}\left(x_{3}\right), \quad \bar{u}_{3}^{a}=\bar{\zeta}^{a}\left(x_{3}\right)-x_{1} \frac{\mathrm{d} \bar{u}_{1}^{a}}{\mathrm{~d} x_{3}}\left(x_{3}\right)-x_{2} \frac{\mathrm{d} \bar{u}_{2}^{a}}{\mathrm{~d} x_{3}}\left(x_{3}\right), \\
\bar{v}_{\alpha}^{a}=\bar{c}\left(x_{3}\right) x_{\alpha}^{\prime R}, \text { that is } \bar{v}_{1}^{a}=-\bar{c}\left(x_{3}\right) x_{2} \text { and } \bar{v}_{2}^{a}=\bar{c}\left(x_{3}\right) x_{1}, \\
\bar{w}_{3}^{a}=0, \\
\bar{u}_{3}^{b}=\bar{u}_{3}^{b}\left(x^{\prime}\right), \quad \bar{u}_{\alpha}^{b}=\bar{\zeta}_{\alpha}^{b}\left(x^{\prime}\right)-x_{3} \frac{\partial \bar{u}_{3}^{b}}{\partial x_{\alpha}}\left(x^{\prime}\right), \\
\bar{v}_{3}^{b}=0, \quad \bar{w}_{\alpha}^{b}=0 .
\end{gathered}
$$

From the above expressions of $\bar{u}^{a}, \bar{v}^{a}, \bar{w}^{a}, \bar{u}^{b}, \bar{v}^{b}, \bar{w}^{b}$, we deduce that:

$$
\begin{gathered}
\left\{\begin{array}{l}
\hat{U}_{\alpha}^{a \varepsilon}\left(\varepsilon^{\frac{3}{2}} x^{\prime}, x_{3}\right)=\bar{u}_{\alpha}^{a}\left(x_{3}\right)+\varepsilon^{\frac{3}{2}} \bar{c}\left(x_{3}\right) x_{\alpha}^{\prime R}+\varepsilon^{3} \bar{w}_{\alpha}^{a}(x), \text { for } x \in \Omega^{a}, \\
\hat{U}_{\alpha}^{b \varepsilon}\left(x^{\prime}, \varepsilon x_{3}\right)=\varepsilon^{\frac{5}{2}}\left(\bar{\zeta}_{\alpha}^{b}\left(x^{\prime}\right)-x_{3} \frac{\partial \bar{u}_{3}^{b}}{\partial x_{\alpha}}\left(x^{\prime}\right)+\varepsilon \bar{v}_{\alpha}^{b}(x)+\varepsilon^{2} \cdot 0\right), \text { for } x \in \Omega^{b},
\end{array}\right. \\
\left\{\begin{array}{l}
\hat{U}_{3}^{a \varepsilon}\left(\varepsilon^{\frac{3}{2}} x^{\prime}, x_{3}\right)=\varepsilon^{\frac{3}{2}}\left(\bar{\zeta}^{a}\left(x_{3}\right)-x_{1} \frac{d \bar{u}_{1}^{a}}{\mathrm{~d} x_{3}}\left(x_{3}\right)-x_{2} \frac{d \bar{u}_{2}^{a}}{\mathrm{~d} x_{3}}\left(x_{3}\right)+\varepsilon^{\frac{3}{2}} \bar{v}_{3}^{a}(x)+\varepsilon^{3} \cdot 0\right), \text { for } x \in \Omega^{a}, \\
\hat{U}_{3}^{b \varepsilon}\left(x^{\prime}, \varepsilon x_{3}\right)=\varepsilon^{\frac{3}{2}}\left(\bar{u}_{3}^{b}\left(x^{\prime}\right)+\varepsilon \cdot 0+\varepsilon^{2} \bar{w}_{3}^{b}(x)\right), \text { for } x \in \Omega^{b} .
\end{array}\right.
\end{gathered}
$$

At least formally, from (2.31), (2.32) and (2.33), it follows that:

$$
\begin{aligned}
& \bar{U}_{\alpha}^{\varepsilon}=O(1) \text { in } \Omega^{a \varepsilon}, \quad \bar{U}_{\alpha}^{\varepsilon}=O\left(\varepsilon^{\frac{5}{2}}\right) \text { in } \Omega^{b \varepsilon}, \\
& \bar{U}_{3}^{\varepsilon}=O\left(\varepsilon^{\frac{3}{2}}\right) \text { in } \Omega^{a \varepsilon}, \quad \bar{U}_{3}^{\varepsilon}=O\left(\varepsilon^{\frac{3}{2}}\right) \text { in } \Omega^{b \varepsilon} .
\end{aligned}
$$


We deduce from these estimates that the main observable displacement is the transversal displacement of the beam. At the junction, the continuity of $\bar{U}^{\varepsilon}$ formally implies that, for $x^{\prime} \in \omega^{a}$ :

$$
\begin{gathered}
\bar{u}_{\alpha}^{a}(0)+\varepsilon^{\frac{3}{2}} \bar{c}(0) x_{\alpha}^{\prime R}+\varepsilon^{3} \bar{w}_{\alpha}^{a}\left(x^{\prime}, 0\right) \simeq \varepsilon^{\frac{5}{2}}\left(\bar{\zeta}_{\alpha}^{b}\left(\varepsilon^{\frac{3}{2}} x^{\prime}\right)-0 \cdot \frac{\partial \bar{u}_{3}^{b}}{\partial x_{\alpha}}\left(\varepsilon^{\frac{3}{2}} x^{\prime}, 0\right)+\varepsilon \bar{v}_{\alpha}^{b}\left(\varepsilon^{\frac{3}{2}} x^{\prime}, 0\right)+\varepsilon^{2} \cdot 0\right), \\
\bar{\zeta}^{a}(0)-x_{1} \frac{\mathrm{d} \bar{u}_{1}^{a}}{\mathrm{~d} x_{3}}(0)-x_{2} \frac{\mathrm{d} \bar{u}_{2}^{a}}{\mathrm{~d} x_{3}}(0)+\varepsilon^{\frac{3}{2}} \bar{v}_{3}^{a}\left(x^{\prime}, 0\right)+\varepsilon^{3} \cdot 0 \simeq \bar{u}_{3}^{b}\left(\varepsilon^{\frac{3}{2}} x^{\prime}\right)+\varepsilon \cdot 0+\varepsilon^{2} \bar{w}_{3}^{b}\left(\varepsilon^{\frac{3}{2}} x^{\prime}, 0\right) .
\end{gathered}
$$

This gives formally:

$$
\begin{gathered}
\bar{u}_{\alpha}^{a}(0)=0, \quad \bar{c}(0)=0, \\
\bar{\zeta}^{a}(0)=\bar{u}_{3}^{b}(0), \quad \frac{\mathrm{d} \bar{u}_{\alpha}^{a}}{\mathrm{~d} x_{3}}(0)=0,
\end{gathered}
$$

which are the six conditions on the junction, a rigourous proof of which is given in Section 5. Moreover we get at the junction the following estimates:

$$
\bar{U}_{\alpha}^{\varepsilon}=O\left(\varepsilon^{\frac{5}{2}}\right), \quad \bar{U}_{3}^{\varepsilon}=O\left(\varepsilon^{\frac{3}{2}}\right) \text { on } J^{\varepsilon} .
$$

Remark 3. We could go further and formally deduce for instance that:

$$
\bar{\zeta}_{\alpha}^{b}(0)=0, \quad \bar{v}_{3}^{a}\left(x^{\prime}, 0\right)=x_{1} \frac{\partial \bar{u}_{3}^{b}}{\partial x_{1}}(0)+x_{2} \frac{\partial \bar{u}_{3}^{b}}{\partial x_{2}}(0)
$$

But these relations have no sense since the solutions are not sufficiently smooth. For instance $\bar{\zeta}_{\alpha}^{b}$ only belongs to $H^{1}\left(\omega^{b}\right)$, and its value $\bar{\zeta}_{\alpha}^{b}(0)$ is not well defined. In contrast, the functions involved in the conditions at the junction have a value in zero, since they belong to the functional space given by the limit problem.

The remaining part of the paper is devoted to the proofs of Theorem 1 and Corollary 1.

\section{The Derivation of the ResCaled PROBlem}

Let us emphasize that we perform different scalings for the respective restrictions of $U \in Y^{\varepsilon}$ to the respective subdomains $\Omega^{a \varepsilon}$ and $\Omega^{b \varepsilon}$, in order to get convenient transmission conditions for their transforms $u^{a}$ and $u^{b}$. We mean that, with the transmission conditions appearing in the definition $(2.1)$ of $\mathcal{Y}^{\varepsilon}$, namely:

$$
u_{\alpha}^{a}\left(x^{\prime}, 0\right)=\varepsilon r^{\varepsilon} u_{\alpha}^{b}\left(r^{\varepsilon} x^{\prime}, 0\right) \text { and } u_{3}^{a}\left(x^{\prime}, 0\right)=u_{3}^{b}\left(r^{\varepsilon} x^{\prime}, 0\right), \quad \text { for a.e. } x^{\prime} \in \omega^{a},
$$

we are able to derive the junction conditions for the limit problem. The derivation of the limit junction conditions seems to be delicate otherwise. Moreover this is the scaling for which the coupling is maximum at the limit, at least for the third component of the displacement.

\subsection{The result of the scaling}

In this subsection, we give the explicit expressions of the source terms and the solution of the rescaled problem (2.3), as functions of the corresponding terms of the initial problem (1.2). An explanation is given in Section 3.2 . 
On the first hand, assuming that $\left(F^{\varepsilon}, G^{\varepsilon}, H^{\varepsilon}\right) \neq(0,0,0)$ (otherwise the problem is trivial), we define $\lambda^{\varepsilon}$ by:

$$
\left\{\begin{array}{l}
\frac{1}{\left(r^{\varepsilon}\right)^{2}} \sum_{\alpha=1}^{2}\left\|F_{\alpha}^{\varepsilon}\right\|_{L^{2}\left(\Omega^{a \varepsilon}\right)}^{2}+\left\|F_{3}^{\varepsilon}\right\|_{L^{2}\left(\Omega^{a \varepsilon}\right)}^{2}+\frac{\varepsilon^{3}}{\left(r^{\varepsilon}\right)^{2}} \sum_{\alpha=1}^{2}\left\|F_{\alpha}^{\varepsilon}\right\|_{L^{2}\left(\Omega^{b \varepsilon}\right)}^{2}+\frac{\varepsilon}{\left(r^{\varepsilon}\right)^{2}}\left\|F_{3}^{\varepsilon}\right\|_{L^{2}\left(\Omega^{b \varepsilon}\right)}^{2} \\
+\left\|G^{\varepsilon}\right\|_{\left(L^{2}\left(\Omega^{a \varepsilon}\right)\right)^{3 \times 3}}^{2}+\frac{\varepsilon^{3}}{\left(r^{\varepsilon}\right)^{2}}\left\|G^{\varepsilon}\right\|_{\left(L^{2}\left(\Omega^{b \varepsilon}\right)\right)^{3 \times 3}}^{2}+\frac{1}{\left(r^{\varepsilon}\right)^{3}} \sum_{\alpha=1}^{2}\left\|H_{\alpha}^{\varepsilon}\right\|_{L^{2}\left(\Sigma^{a \varepsilon}\right)}^{2}+\frac{1}{r^{\varepsilon}}\left\|H_{3}^{\varepsilon}\right\|_{L^{2}\left(\Sigma^{a \varepsilon}\right)}^{2} \\
+\frac{\varepsilon^{2}}{\left(r^{\varepsilon}\right)^{2}} \sum_{\alpha=1}^{2}\left\|H_{\alpha}^{\varepsilon}\right\|_{L^{2}\left(T^{b \varepsilon} \cup B^{b \varepsilon}\right)}^{2}+\frac{1}{\left(r^{\varepsilon}\right)^{2}}\left\|H_{3}^{\varepsilon}\right\|_{L^{2}\left(T^{b \varepsilon} \cup B^{b \varepsilon}\right)}^{2}=\left(\frac{r^{\varepsilon}}{\lambda^{\varepsilon}}\right)^{2}
\end{array}\right.
$$

Then we set:

$$
\begin{aligned}
& \left\{\begin{array}{lll}
f_{\alpha}^{a \varepsilon}(x)=\frac{\lambda^{\varepsilon}}{r^{\varepsilon}} F_{\alpha}^{\varepsilon}\left(r^{\varepsilon} x^{\prime}, x_{3}\right), & f_{3}^{a \varepsilon}(x)=\lambda^{\varepsilon} F_{3}^{\varepsilon}\left(r^{\varepsilon} x^{\prime}, x_{3}\right), & \text { for } x \in \Omega^{a} \\
f_{\alpha}^{b \varepsilon}(x)=\lambda^{\varepsilon} \frac{\varepsilon^{2}}{\left(r^{\varepsilon}\right)^{2}} F_{\alpha}^{\varepsilon}\left(x^{\prime}, \varepsilon x_{3}\right), & f_{3}^{b \varepsilon}(x)=\lambda^{\varepsilon} \frac{\varepsilon}{\left(r^{\varepsilon}\right)^{2}} F_{3}^{\varepsilon}\left(x^{\prime}, \varepsilon x_{3}\right), & \text { for } x \in \Omega^{b}
\end{array}\right. \\
& g^{a \varepsilon}(x)=\lambda^{\varepsilon} G^{\varepsilon}\left(r^{\varepsilon} x^{\prime}, x_{3}\right), \text { for } x \in \Omega^{a}, \quad g^{b \varepsilon}(x)=\lambda^{\varepsilon} \frac{\varepsilon^{2}}{\left(r^{\varepsilon}\right)^{2}} G^{\varepsilon}\left(x^{\prime}, \varepsilon x_{3}\right), \text { for } x \in \Omega^{b}
\end{aligned}
$$

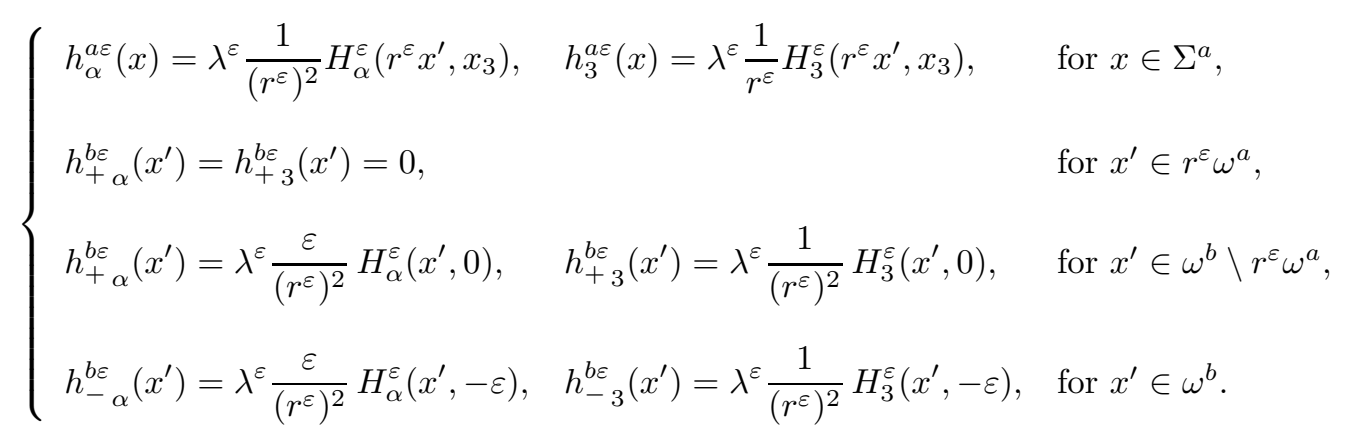

Note that $h_{+}^{b \varepsilon}=0$ on $r^{\varepsilon} \omega^{a}$, since there is no contribution of $H^{\varepsilon}$ on $J^{\varepsilon}$.

On the other hand, for any $U \in Y^{\varepsilon}$, we define the rescaled function $u=\left(u^{a}, u^{b}\right)$ by:

$$
\begin{gathered}
u_{\alpha}^{a}(x)=\lambda^{\varepsilon} r^{\varepsilon} U_{\alpha}\left(r^{\varepsilon} x^{\prime}, x_{3}\right), \quad u_{3}^{a}(x)=\lambda^{\varepsilon} U_{3}\left(r^{\varepsilon} x^{\prime}, x_{3}\right), \text { for } x \in \Omega^{a}, \\
u_{\alpha}^{b}(x)=\lambda^{\varepsilon} \frac{1}{\varepsilon} U_{\alpha}\left(x^{\prime}, \varepsilon x_{3}\right), \quad u_{3}^{b}(x)=\lambda^{\varepsilon} U_{3}\left(x^{\prime}, \varepsilon x_{3}\right), \text { for } x \in \Omega^{b} .
\end{gathered}
$$

Remark 4. Let us observe that the rescaled source terms are bounded, but not strongly converging to zero, since, by definition of $\lambda^{\varepsilon}$ (see (3.2)) and by (3.3) to (3.5):

$$
\begin{aligned}
& \left\|f^{a \varepsilon}\right\|_{\left(L^{2}\left(\Omega^{a}\right)\right)^{3}}^{2}+\left\|f^{b \varepsilon}\right\|_{\left(L^{2}\left(\Omega^{b}\right)\right)^{3}}^{2}+\left\|g^{a \varepsilon}\right\|_{\left(L^{2}\left(\Omega^{a}\right)\right)^{3 \times 3}}^{2}+\left\|g^{b \varepsilon}\right\|_{\left(L^{2}\left(\Omega^{b}\right)\right)^{3 \times 3}}^{2} \\
& +\left\|h^{a \varepsilon}\right\|_{\left(L^{2}\left(\Sigma^{a}\right)\right)^{3}}^{2}+\left\|h_{+}^{b \varepsilon}\right\|_{\left(L^{2}\left(\omega^{b}\right)\right)^{3}}^{2}+\left\|h_{-}^{b \varepsilon}\right\|_{\left(L^{2}\left(\omega^{b}\right)\right)^{3}}^{2}=1 .
\end{aligned}
$$




\subsection{The derivation of the scaling}

Let us consider the possible scalings for the solution $\bar{U}^{\varepsilon}$ and test function $U$. If, instead of a multidomain, one considers a single thin cylinder, the natural scaling is (see $[21,27,28]$ ):

$$
u_{\alpha}(x)=r^{\varepsilon} U_{\alpha}\left(r^{\varepsilon} x^{\prime}, x_{3}\right), u_{3}(x)=U_{3}\left(r^{\varepsilon} x^{\prime}, x_{3}\right), \text { for } x \in \Omega^{a},
$$

while for a single plate, the natural scaling is (see $[5,7])$ :

$$
u_{\alpha}(x)=U_{\alpha}\left(x^{\prime}, \varepsilon x_{3}\right), u_{3}(x)=\varepsilon U_{3}\left(x^{\prime}, \varepsilon x_{3}\right), \text { for } x \in \Omega^{b}
$$

For the multidomain made of the union of the beam and the plate, the idea is to consider different coefficients of normalization, $\lambda^{a \varepsilon}$ and $\lambda^{b \varepsilon}$, for $\Omega^{a \varepsilon}$ and $\Omega^{b \varepsilon}$ respectively, that is we set:

$$
\begin{aligned}
& u_{\alpha}^{a}(x)=\lambda^{a \varepsilon} r^{\varepsilon} U_{\alpha}\left(r^{\varepsilon} x^{\prime}, x_{3}\right), \quad u_{3}^{a}(x)=\lambda^{a \varepsilon} U_{3}\left(r^{\varepsilon} x^{\prime}, x_{3}\right), \quad \text { for } x \in \Omega^{a}, \\
& u_{\alpha}^{b}(x)=\lambda^{b \varepsilon} U_{\alpha}\left(x^{\prime}, \varepsilon x_{3}\right), \quad u_{3}^{b}(x)=\lambda^{b \varepsilon} \varepsilon U_{3}\left(x^{\prime}, \varepsilon x_{3}\right), \quad \text { for } x \in \Omega^{b} .
\end{aligned}
$$

Then one has, with $e^{a \varepsilon}, e^{b \varepsilon}$ defined in $(2.2)$ :

$$
e(U)\left(r^{\varepsilon} x^{\prime}, x_{3}\right)=\frac{1}{\lambda^{a \varepsilon}} e^{a \varepsilon}\left(u^{a \varepsilon}\right)(x) \text { for } x \in \Omega^{a} \text { and } e(U)\left(x^{\prime}, \varepsilon x_{3}\right)=\frac{1}{\lambda^{b \varepsilon}} e^{b \varepsilon}\left(u^{b \varepsilon}\right)(x) \text { for } x \in \Omega^{b},
$$

and it is easy to check that the variational equality in (1.2) reads, once each integral is written on the corresponding fixed domain:

$$
\left\{\begin{array}{l}
\frac{\left(r^{\varepsilon}\right)^{2}}{\left(\lambda^{a \varepsilon}\right)^{2}} \int_{\Omega^{a}}\left[A^{a} e^{a \varepsilon}\left(\bar{u}^{a \varepsilon}\right), e^{a \varepsilon}\left(u^{a}\right)\right] \mathrm{d} x+k^{\varepsilon} \frac{\varepsilon}{\left(\lambda^{b \varepsilon}\right)^{2}} \int_{\Omega^{b}}\left[A^{b} e^{b \varepsilon}\left(\bar{u}^{b \varepsilon}\right), e^{b \varepsilon}\left(u^{b}\right)\right] \mathrm{d} x \\
=\frac{1}{\lambda^{a \varepsilon}}\left(\sum_{\alpha=1}^{2} \int_{\Omega^{a}} r^{\varepsilon} F_{\alpha}^{\varepsilon}\left(r^{\varepsilon} x^{\prime}, x_{3}\right) u_{\alpha}^{a}(x) \mathrm{d} x+\int_{\Omega^{a}}\left(r^{\varepsilon}\right)^{2} F_{3}^{\varepsilon}\left(r^{\varepsilon} x^{\prime}, x_{3}\right) u_{3}^{a}(x) \mathrm{d} x\right) \\
+\frac{1}{\lambda^{b \varepsilon}}\left(\sum_{\alpha=1}^{2} \int_{\Omega^{b}} \varepsilon F_{\alpha}^{\varepsilon}\left(x^{\prime}, \varepsilon x_{3}\right) u_{\alpha}^{b}(x) \mathrm{d} x+\int_{\Omega^{b}} F_{3}^{\varepsilon}\left(x^{\prime}, \varepsilon x_{3}\right) u_{3}^{b}(x) \mathrm{d} x\right) \\
+\frac{\left(r^{\varepsilon}\right)^{2}}{\lambda^{a \varepsilon}} \int_{\Omega^{a}}\left[G^{\varepsilon}\left(r^{\varepsilon} x^{\prime}, x_{3}\right), e^{a \varepsilon}\left(u^{a}\right)\right] \mathrm{d} x+\frac{\varepsilon}{\lambda^{b \varepsilon}} \int_{\Omega^{b}}\left[G^{\varepsilon}\left(x^{\prime}, \varepsilon x_{3}\right), e^{b \varepsilon}\left(u^{b}\right)\right] \mathrm{d} x \\
+\frac{1}{\lambda^{a \varepsilon}}\left(\sum_{\alpha=1}^{2} \int_{\Sigma^{a}} H_{\alpha}^{\varepsilon}\left(r^{\varepsilon} x^{\prime}, x_{3}\right) u_{\alpha}^{a}(x) \mathrm{d} \sigma+\int_{\Sigma^{a}} r^{\varepsilon} H_{3}^{\varepsilon}\left(r^{\varepsilon} x^{\prime}, x_{3}\right) u_{3}^{a}(x) \mathrm{d} \sigma\right) \\
+\frac{1}{\lambda^{b \varepsilon}}\left(\sum_{\alpha=1}^{2} \int_{\omega^{b} \backslash r^{\varepsilon} \omega^{a}} H_{\alpha}^{\varepsilon}\left(x^{\prime}, 0\right) u_{\alpha}^{b}\left(x^{\prime}, 0\right) \mathrm{d} x^{\prime}+\int_{\omega^{b} \backslash r^{\varepsilon} \omega^{a}} \frac{1}{\varepsilon} H_{3}^{\varepsilon}\left(x^{\prime}, 0\right) u_{3}^{b}\left(x^{\prime}, 0\right) \mathrm{d} x^{\prime}\right) \\
+\frac{1}{\lambda^{b \varepsilon}}\left(\sum_{\alpha=1}^{2} \int_{\omega^{b}} H_{\alpha}^{\varepsilon}\left(x^{\prime},-\varepsilon\right) u_{\alpha}^{b}\left(x^{\prime},-1\right) \mathrm{d} x^{\prime}+\int_{\omega^{b}} \frac{1}{\varepsilon} H_{3}^{\varepsilon}\left(x^{\prime},-\varepsilon\right) u_{3}^{b}\left(x^{\prime},-1\right) \mathrm{d} x^{\prime}\right) .
\end{array}\right.
$$


We decide to choose $\lambda^{a \varepsilon}=\varepsilon \lambda^{b \varepsilon}$, so that the junction condition written for $\left(u^{a}, u^{b}\right)$ reads: for almost every $x^{\prime}$ in $\omega^{a}$, one has:

$$
u_{\alpha}^{a}\left(x^{\prime}, 0\right)=\frac{\lambda^{a \varepsilon}}{\lambda^{b \varepsilon}} r^{\varepsilon} u_{\alpha}^{b}\left(r^{\varepsilon} x^{\prime}, 0\right)=\varepsilon r^{\varepsilon} u_{\alpha}^{b}\left(r^{\varepsilon} x^{\prime}, 0\right) \text { and } u_{3}^{a}\left(x^{\prime}, 0\right)=\frac{\lambda^{a \varepsilon}}{\lambda^{b \varepsilon}} \frac{1}{\varepsilon} u_{3}^{b}\left(r^{\varepsilon} x^{\prime}, 0\right)=u_{3}^{b}\left(r^{\varepsilon} x^{\prime}, 0\right)
$$

(see also the beginning of Sect. 3). Then, after dividing by $\left(r^{\varepsilon}\right)^{2} /\left(\lambda^{a \varepsilon}\right)^{2}$, writing $\lambda^{\varepsilon}$ instead of $\lambda^{a \varepsilon}$, for simplicity, and defining the rescaled source terms by $(3.3),(3.4),(3.5)$, the equality (3.8) is exactly the variational equality in (2.3). Finally, we recall that the particular choice of $\lambda^{\varepsilon}$ given in (3.2) makes the source terms bounded, but not strongly converging to zero (see also Rem. 4).

Remark 5. Since the left-hand side of (3.8) is another way of writing $\int_{\Omega^{\varepsilon}}\left[A e\left(\bar{U}^{\varepsilon}\right), e(U)\right] \mathrm{d} x$, it follows that:

$$
\left(\frac{r^{\varepsilon}}{\lambda^{\varepsilon}}\right)^{2}\left(\int_{\Omega^{a}}\left[A^{a} e^{a \varepsilon}\left(\bar{u}^{a \varepsilon}\right), e^{a \varepsilon}\left(\bar{u}^{a \varepsilon}\right)\right] \mathrm{d} x+q^{\varepsilon} \int_{\Omega^{b}}\left[A^{b} e^{b \varepsilon}\left(\bar{u}^{b \varepsilon}\right), e^{b \varepsilon}\left(\bar{u}^{b \varepsilon}\right)\right] \mathrm{d} x\right)=\int_{\Omega^{\varepsilon}}\left[A e\left(\bar{U}^{\varepsilon}\right), e\left(\bar{U}^{\varepsilon}\right)\right] \mathrm{d} x
$$

which gives the definition of the renormalized energy in (2.26). In [15], we took $\lambda^{\varepsilon}=r^{\varepsilon}$, since the initial problem (1.2) was supposed to be suitably normalized.

\section{The A PRIORI ESTIMATES AND THE COMPACTNESS ARGUMENTS}

\subsection{A priori estimates}

In the following, we denote by $C$ any positive constant which does not depend on $\varepsilon$ and we write $\bar{e}^{a \varepsilon}$ (resp. $\bar{e}^{b \varepsilon}$ ) for $e^{a \varepsilon}\left(\bar{u}^{a \varepsilon}\right)\left(\right.$ resp. $\left.e^{b \varepsilon}\left(\bar{u}^{b \varepsilon}\right)\right)$. Taking $u=\bar{u}^{\varepsilon}=\left(\bar{u}^{a \varepsilon}, \bar{u}^{b \varepsilon}\right)$ as test function in (2.3), we get:

$$
\left\{\begin{array}{l}
\int_{\Omega^{a}}\left[A^{a} \bar{e}^{a \varepsilon}, \bar{e}^{a \varepsilon}\right] \mathrm{d} x+q^{\varepsilon} \int_{\Omega^{b}}\left[A^{b} \bar{e}^{b \varepsilon}, \bar{e}^{b \varepsilon}\right] \mathrm{d} x \\
=\int_{\Omega^{a}} f^{a \varepsilon} \cdot \bar{u}^{a \varepsilon} \mathrm{d} x+\int_{\Omega^{b}} f^{b \varepsilon} \cdot \bar{u}^{b \varepsilon} \mathrm{d} x+\int_{\Omega^{a}}\left[g^{a \varepsilon}, \bar{e}^{a \varepsilon}\right] \mathrm{d} x+\int_{\Omega^{b}}\left[g^{b \varepsilon}, \bar{e}^{b \varepsilon}\right] \mathrm{d} x \\
+\int_{\Sigma^{a}} h^{a \varepsilon} \cdot \bar{u}^{a \varepsilon} \mathrm{d} \sigma+\int_{\omega^{b}}\left(h_{+}^{b \varepsilon} \cdot \bar{u}_{\mid x_{3}=0}^{b \varepsilon}+h_{-}^{b \varepsilon} \cdot \bar{u}_{\mid x_{3}=-1}^{b \varepsilon}\right) \mathrm{d} x^{\prime} .
\end{array}\right.
$$

From Korn's inequality, since $\bar{u}^{a \varepsilon}$ vanishes on $T^{a}$ and $\bar{u}^{b \varepsilon}$ vanishes on $\Sigma^{b}$, we get for $\varepsilon \leq 1$ and $r^{\varepsilon} \leq 1$ :

$$
\begin{aligned}
\left\|\bar{u}^{a \varepsilon}\right\|_{\left(H^{1}\left(\Omega^{a}\right)\right)^{3}} & \leq C\left\|e\left(\bar{u}^{a \varepsilon}\right)\right\|_{\left(L^{2}\left(\Omega^{a}\right)\right)^{3 \times 3}} \leq C\left\|\bar{e}^{a \varepsilon}\right\|_{\left(L^{2}\left(\Omega^{a}\right)\right)^{3 \times 3}}, \\
\left\|\bar{u}^{b \varepsilon}\right\|_{\left(H^{1}\left(\Omega^{b}\right)\right)^{3}} & \leq C\left\|e\left(\bar{u}^{b \varepsilon}\right)\right\|_{\left(L^{2}\left(\Omega^{b}\right)\right)^{3 \times 3}} \leq C\left\|\bar{e}^{b \varepsilon}\right\|_{\left(L^{2}\left(\Omega^{b}\right)\right)^{3 \times 3}},
\end{aligned}
$$

and, by continuity of the trace mapping:

$$
\begin{gathered}
\left\|\bar{u}^{a \varepsilon}\right\|_{\left(L^{2}\left(\Sigma^{a}\right)\right)^{3}} \leq C\left\|\bar{u}^{a \varepsilon}\right\|_{\left(H^{1}\left(\Omega^{a}\right)\right)^{3}}, \\
\left\|\bar{u}_{\mid x_{3}=0}^{b \varepsilon}\right\|_{\left(L^{2}\left(\omega^{b}\right)\right)^{3}}+\left\|\bar{u}_{\mid x_{3}=-1}^{b \varepsilon}\right\|_{\left(L^{2}\left(\omega^{b}\right)\right)^{3}} \leq C\left\|\bar{u}^{b \varepsilon}\right\|_{\left(H^{1}\left(\Omega^{b}\right)\right)^{3}}
\end{gathered}
$$

By using the above inequalities, the coercivity of $A^{a}$ and $A^{b}$ and the boundedness of the source terms (see (2.7) 
to (2.12) and Rem. 4), it follows from (4.1) that

$$
\begin{aligned}
& C\left\|\bar{e}^{a \varepsilon}\right\|_{\left(L^{2}\left(\Omega^{a}\right)\right)^{3 \times 3}}^{2}+C q^{\varepsilon}\left\|\bar{e}^{b \varepsilon}\right\|_{\left(L^{2}\left(\Omega^{b}\right)\right)^{3 \times 3}}^{2} \\
& \leq\left(\left\|f^{a \varepsilon}\right\|_{\left(L^{2}\left(\Omega^{a}\right)\right)^{3}}+\left\|g^{a \varepsilon}\right\|_{\left(L^{2}\left(\Omega^{a}\right)\right)^{3 \times 3}}+\left\|h^{a \varepsilon}\right\|_{\left(L^{2}\left(\Sigma^{a}\right)\right)^{3}}\right)\left\|\bar{e}^{a \varepsilon}\right\|_{\left(L^{2}\left(\Omega^{a}\right)\right)^{3 \times 3}} \\
& +\left(\left\|f^{b \varepsilon}\right\|_{\left(L^{2}\left(\Omega^{b}\right)\right)^{3}}+\left\|g^{b \varepsilon}\right\|_{\left(L^{2}\left(\Omega^{a}\right)\right)^{3 \times 3}}+\left\|h_{+}^{b \varepsilon}\right\|_{\left(L^{2}\left(\omega^{b}\right)\right)^{3}}+\left\|h_{-}^{b \varepsilon}\right\|_{\left(L^{2}\left(\omega^{b}\right)\right)^{3}}\right)\left\|\bar{e}^{b \varepsilon}\right\|_{\left(L^{2}\left(\Omega^{b}\right)\right)^{3 \times 3}} \\
& \leq C\left(\left\|\bar{e}^{a \varepsilon}\right\|_{\left(L^{2}\left(\Omega^{a}\right)\right)^{3 \times 3}}+\left\|\bar{e}^{b \varepsilon}\right\|_{\left(L^{2}\left(\Omega^{b}\right)\right)^{3 \times 3}}\right) .
\end{aligned}
$$

- If $q^{\varepsilon}$ is bounded from below by some positive constant, that is if $q$ defined in (2.5) is equal to some positive number or to $+\infty$, it follows that $\bar{e}^{a \varepsilon}$ is bounded in $\left(L^{2}\left(\Omega^{a}\right)\right)^{3 \times 3}$ and $\bar{e}^{b \varepsilon}$ is bounded in $\left(L^{2}\left(\Omega^{b}\right)\right)^{3 \times 3}$. Then, from Korn's inequality, it results that $\bar{u}^{a \varepsilon}$ is bounded in $\left(H^{1}\left(\Omega^{a}\right)\right)^{3}$ and $\bar{u}^{b \varepsilon}$ is bounded in $\left(H^{1}\left(\Omega^{b}\right)\right)^{3}$. Moreover, in the particular case where $q=+\infty, \bar{e}^{b \varepsilon}$ tends to zero (strongly) in $\left(L^{2}\left(\Omega^{b}\right)\right)^{3 \times 3}$ and $\bar{u}^{b \varepsilon}$ tends to zero (strongly) in $\left(H^{1}\left(\Omega^{b}\right)\right)^{3}$.

- Otherwise, i.e. if $q^{\varepsilon}$ tends to zero, we define $\tilde{u}^{\varepsilon}$ by:

$$
\tilde{u}^{\varepsilon}=\left(\tilde{u}^{a \varepsilon}, \tilde{u}^{b \varepsilon}\right)=q^{\varepsilon} \bar{u}^{\varepsilon}=q^{\varepsilon}\left(\bar{u}^{a \varepsilon}, \bar{u}^{b \varepsilon}\right) .
$$

It is clear that $\tilde{u}^{\varepsilon}$ solves:

$$
\left\{\begin{array}{l}
\tilde{u}^{\varepsilon} \in \mathcal{Y}^{\varepsilon} \text { and } \forall u \in \mathcal{Y}^{\varepsilon} \\
\frac{1}{q^{\varepsilon}} \int_{\Omega^{a}}\left[A^{a} e^{a \varepsilon}\left(\tilde{u}^{a \varepsilon}\right), e^{a \varepsilon}\left(u^{a}\right)\right] \mathrm{d} x+\int_{\Omega^{b}}\left[A^{b} e^{b \varepsilon}\left(\tilde{u}^{b \varepsilon}\right), e^{b \varepsilon}\left(u^{b}\right)\right] \mathrm{d} x \\
=\int_{\Omega^{a}} f^{a \varepsilon} \cdot u^{a} \mathrm{~d} x+\int_{\Omega^{b}} f^{b \varepsilon} \cdot u^{b} \mathrm{~d} x+\int_{\Omega^{a}}\left[g^{a \varepsilon}, e^{a \varepsilon}\left(u^{a}\right)\right] \mathrm{d} x+\int_{\Omega^{b}}\left[g^{b \varepsilon}, e^{b \varepsilon}\left(u^{b}\right)\right] \mathrm{d} x \\
+\int_{\Sigma^{a}} h^{a \varepsilon} \cdot u^{a} \mathrm{~d} \sigma+\int_{\omega^{b}}\left(h_{+}^{b \varepsilon} \cdot u_{\mid x_{3}=0}^{b}+h_{-}^{b \varepsilon} \cdot u_{\mid x_{3}=-1}^{b}\right) \mathrm{d} x^{\prime} .
\end{array}\right.
$$

Taking $u=\tilde{u}^{\varepsilon}$ as test function in (4.3), it is easy to prove (as we have done in the case $q^{\varepsilon} \geq C>0$ ) that $\tilde{e}^{a \varepsilon}=e^{a \varepsilon}\left(\tilde{u}^{a \varepsilon}\right)=q^{\varepsilon} \bar{e}^{a \varepsilon}$ tends to zero in $\left(L^{2}\left(\Omega^{a}\right)\right)^{3 \times 3}, \tilde{e}^{b \varepsilon}=e^{b \varepsilon}\left(\tilde{u}^{b \varepsilon}\right)=q^{\varepsilon} \bar{e}^{b \varepsilon}$ is bounded in $\left(L^{2}\left(\Omega^{b}\right)\right)^{3 \times 3}$, $\tilde{u}^{a \varepsilon}=q^{\varepsilon} \bar{u}^{a \varepsilon}$ tends to zero in $\left(H^{1}\left(\Omega^{a}\right)\right)^{3}$ and $\tilde{u}^{b \varepsilon}=q^{\varepsilon} \bar{u}^{b \varepsilon}$ is bounded in $\left(H^{1}\left(\Omega^{b}\right)\right)^{3}$.

\subsection{Compactness arguments}

- If $q^{\varepsilon}$ tends to $q$ with $0<q \leq+\infty$, it results from the a priori estimates that there exist $\bar{u}=\left(\bar{u}^{a}, \bar{u}^{b}\right)$ in $\left(H^{1}\left(\Omega^{a}\right)\right)^{3} \times\left(H^{1}\left(\Omega^{b}\right)\right)^{3}$ and $\bar{e}=\left(\bar{e}^{a}, \bar{e}^{b}\right)$ in $\left(L^{2}\left(\Omega^{a}\right)\right)^{3 \times 3} \times\left(L^{2}\left(\Omega^{b}\right)\right)^{3 \times 3}$ such that:

$$
\begin{gathered}
\bar{u}^{\varepsilon}=\left(\bar{u}^{a \varepsilon}, \bar{u}^{b \varepsilon}\right) \rightarrow \bar{u}=\left(\bar{u}^{a}, \bar{u}^{b}\right) \text { weakly in }\left(H^{1}\left(\Omega^{a}\right)\right)^{3} \times\left(H^{1}\left(\Omega^{b}\right)\right)^{3}, \\
\bar{e}^{\varepsilon}=\left(\bar{e}^{a \varepsilon}, \bar{e}^{b \varepsilon}\right) \rightarrow \bar{e}=\left(\bar{e}^{a}, \bar{e}^{b}\right) \text { weakly in }\left(L^{2}\left(\Omega^{a}\right)\right)^{3 \times 3} \times\left(L^{2}\left(\Omega^{b}\right)\right)^{3 \times 3} .
\end{gathered}
$$

Clearly $\bar{u}^{a}=0$ on $T^{a}, \bar{u}^{b}=0$ on $\Sigma^{b}$ and $\bar{e}^{a}, \bar{e}^{b}$ are symmetric matrices. Moreover, from the boundedness of $\bar{e}^{\varepsilon}=\left(\bar{e}^{a \varepsilon}, \bar{e}^{b \varepsilon}\right)$ and a classical semicontinuity argument, we get that $\bar{u}^{a}$ is a Bernoulli-Navier displacement and $\bar{u}^{b}$ is a Kirchhoff-Love displacement:

$$
e_{\alpha \beta}\left(\bar{u}^{a}\right)=0 \text { and } e_{\alpha 3}\left(\bar{u}^{a}\right)=0, \quad e_{\alpha 3}\left(\bar{u}^{b}\right)=0 \text { and } e_{33}\left(\bar{u}^{b}\right)=0,
$$


which, combined with the constraints $\bar{u}^{a}=0$ on $T^{a}, \bar{u}^{b}=0$ on $\Sigma^{b}$, is equivalent to (see [20]):

$$
\begin{aligned}
& \bar{u}^{a} \in\left(H^{2}(0,1)\right)^{2} \times H^{1}\left(\Omega^{a}\right), \quad \bar{u}_{\alpha}^{a}(1)=\frac{\mathrm{d} \bar{u}_{\alpha}^{a}}{\mathrm{~d} x_{3}}(1)=0, \\
& \exists \bar{\zeta}^{a} \in H^{1}(0,1), \quad \bar{\zeta}^{a}(1)=0, \quad \bar{u}_{3}^{a}=\bar{\zeta}^{a}-x_{1} \frac{\mathrm{d} \bar{u}_{1}^{a}}{\mathrm{~d} x_{3}}-x_{2} \frac{\mathrm{d} \bar{u}_{2}^{a}}{\mathrm{~d} x_{3}} \\
& \bar{u}^{b} \in \mathcal{U}^{b} .
\end{aligned}
$$

Moreover one can prove as in [28] that there exist $\left(\bar{v}^{a}, \bar{w}^{a}\right)$ and $\left(\bar{v}^{b}, \bar{w}^{b}\right)$ such that $\bar{e}^{a}=e^{a}\left(\bar{u}^{a}, \bar{v}^{a}, \bar{w}^{a}\right)$ and $\bar{e}^{b}=e^{b}\left(\bar{u}^{b}, \bar{v}^{b}, \bar{w}^{b}\right)$ (see the definitions of $e^{a}$ and $e^{b}$ in (2.6)) and such that:

$$
\begin{array}{ll}
\bar{v}^{a} \in\left(H^{1}\left(\Omega^{a}\right)\right)^{2} \times L^{2}\left(0,1 ; H^{1}\left(\omega^{a}\right)\right), \quad \exists \bar{c} \in H^{1}(0,1), & \bar{c}(1)=0, \quad \bar{v}_{1}^{a}=-\bar{c} x_{2}, \quad \bar{v}_{2}^{a}=\bar{c} x_{1}, \\
& \int_{\omega^{a}} \bar{v}_{3}^{a}\left(x^{\prime}, x_{3}\right) \mathrm{d} x^{\prime}=0, \quad \text { for a.e. } x_{3} \in(0,1), \\
\bar{w}^{a} \in \mathcal{W}^{a}, \quad \bar{v}^{b} \in \mathcal{V}^{b}, \quad \bar{w}^{b} \in \mathcal{W}^{b}, &
\end{array}
$$

and suitable expressions of $\bar{u}^{a \varepsilon}$ (resp. $\left.\bar{u}^{b \varepsilon}\right)$ tend to $\left(\bar{v}^{a}, \bar{w}^{a}\right)\left(\right.$ resp. $\left.\left(\bar{v}^{b}, \bar{w}^{b}\right)\right)$. For the convenience of the reader, the proof of this fact is given in the Appendix (see Sect. 8.1). In particular, $\bar{v}^{a}$ defines some $\bar{c} \in H^{1}(0,1)$ with $\bar{c}(1)=0$, which is actually the limit in $L^{2}(0,1)$ of $\bar{c}^{\varepsilon}$ given by

$$
\bar{c}^{\varepsilon}\left(x_{3}\right)=\frac{\int_{\omega^{a}}\left(x_{1} \bar{u}_{2}^{a \varepsilon}\left(x^{\prime}, x_{3}\right)-x_{2} \bar{u}_{1}^{a \varepsilon}\left(x^{\prime}, x_{3}\right)\right) \mathrm{d} x^{\prime}}{r^{\varepsilon} \int_{\omega^{a}}\left(x_{1}^{2}+x_{2}^{2}\right) \mathrm{d} x^{\prime}} .
$$

In conclusion we have proved (2.13), (2.14).

In the particular case $q=+\infty$, we have already noticed (see the a priori estimates) that:

$$
\bar{u}^{b \varepsilon} \rightarrow \bar{u}^{b}=0 \text { strongly in }\left(H^{1}\left(\Omega^{b}\right)\right)^{3} \text { and } \bar{e}^{b \varepsilon} \rightarrow \bar{e}^{b}=0 \text { strongly in }\left(L^{2}\left(\Omega^{b}\right)\right)^{3 \times 3}
$$

that is we have proved (2.16), (2.17).

- If $q^{\varepsilon}$ tends to zero, it results from the a priori estimates that:

$$
\left\{\begin{array}{l}
q^{\varepsilon} \bar{u}^{a \varepsilon} \rightarrow 0 \text { strongly in }\left(H^{1}\left(\Omega^{a}\right)\right)^{3}, \quad q^{\varepsilon} \bar{u}^{b \varepsilon} \rightarrow \bar{u}^{b} \text { weakly in }\left(H^{1}\left(\Omega^{b}\right)\right)^{3}, \\
q^{\varepsilon} \bar{e}^{a \varepsilon} \rightarrow 0 \text { strongly in }\left(L^{2}\left(\Omega^{a}\right)\right)^{3 \times 3}, \quad q^{\varepsilon} \bar{e}^{b \varepsilon} \rightarrow \bar{e}^{b} \text { weakly in }\left(L^{2}\left(\Omega^{b}\right)\right)^{3 \times 3}
\end{array}\right.
$$

for some $\bar{u}^{b} \in \mathcal{U}^{b}$ and some symmetric matrix $\bar{e}^{b} \in\left(L^{2}\left(\Omega^{b}\right)\right)^{3 \times 3}$. Again (see the App., Sect. 8.1), there exists $\left(\bar{v}^{b}, \bar{w}^{b}\right)$ in $\mathcal{V}^{b} \times \mathcal{W}^{b}$, which are limits of suitable expressions of $\bar{u}^{b \varepsilon}$ and such that $\bar{e}^{b}=e^{b}\left(\bar{u}^{b}, \bar{v}^{b}, \bar{w}^{b}\right)=e^{b}\left(\bar{z}^{b}\right)$. In other words, we have proved (2.21), (2.22). 


\section{The Limit CONStRaints that ARE DUe to THE JUNCTION}

As for the limit constraints, it remains to prove that

1) $\bar{u}_{\alpha}^{a}(0)=0$,

2) $\bar{u}_{3}^{a}\left(x^{\prime}, 0\right)=\bar{u}_{3}^{b}(0)$, which is equivalent to $\bar{\zeta}^{a}(0)=\bar{u}_{3}^{b}(0)$ and $\frac{\mathrm{d} \bar{u}_{\alpha}^{a}}{\mathrm{~d} x_{3}}(0)=0$,

3) $\bar{c}(0)=0$,

since the above three conditions give $\bar{u}_{\alpha}^{a} \in\left(H_{0}^{2}(0,1)\right)^{2}$ and $\bar{c} \in H_{0}^{1}(0,1)$, so that $\bar{u}^{a} \in \mathcal{U}^{a}$ and $\bar{v}^{a} \in \mathcal{V}^{a}$. These limit constraints are derived below.

\subsection{Proof of $\bar{u}_{\alpha}^{a}(\mathbf{0})=\mathbf{0}$}

The fact that $\bar{u}_{\alpha}^{a}(0)=0$ results from the following easy lemma:

Lemma 1. Assume that $\left\{u^{b \varepsilon}\right\}_{\varepsilon}$ is bounded in $L^{2}\left(\omega^{b}\right)$. Then $\left\{r^{\varepsilon} u^{b \varepsilon}\left(r^{\varepsilon} .\right)\right\}_{\varepsilon}$ is bounded in $L^{2}\left(\omega^{a}\right)$, for every $\omega^{a}$ such that $r^{\varepsilon} \omega^{a} \subset \omega^{b}$, for any $\varepsilon$.

Proof. We have:

$$
\int_{\omega^{a}}\left|r^{\varepsilon} u^{b \varepsilon}\left(r^{\varepsilon} x^{\prime}\right)\right|^{2} \mathrm{~d} x^{\prime}=\int_{r^{\varepsilon} \omega^{a}}\left|u^{b \varepsilon}\left(x^{\prime}\right)\right|^{2} \mathrm{~d} x^{\prime} \leq \int_{\omega^{b}}\left|u^{b \varepsilon}\left(x^{\prime}\right)\right|^{2} \mathrm{~d} x^{\prime} \leq C .
$$

Application. If $q \neq 0$, we write the junction condition for $\bar{u}_{\alpha}^{\varepsilon}$ as:

$$
\bar{u}_{\alpha}^{a \varepsilon}\left(x^{\prime}, 0\right)=\varepsilon r^{\varepsilon} \bar{u}_{\alpha}^{b \varepsilon}\left(r^{\varepsilon} x^{\prime}, 0\right), \quad \text { for } \text { a.e. } x^{\prime} \in \omega^{a} .
$$

The left-hand side tends to $\bar{u}_{\alpha}^{a}\left(x^{\prime}, 0\right)=\bar{u}_{\alpha}^{a}(0)$ in $L^{2}\left(\omega^{a}\right)$. The right-hand side tends to zero in this space, by Lemma 1 , since $\bar{u}_{\alpha}^{b \varepsilon}(., 0)$ is bounded in $L^{2}\left(\omega^{b}\right)$, so that $\bar{u}_{\alpha}^{a}(0)=0$. If $q=0$, the same proof applies to $\tilde{u}^{\varepsilon}=q^{\varepsilon} \bar{u}^{\varepsilon}$.

5.2. Proof of $\bar{u}_{3}^{a}\left(x^{\prime}, 0\right)=\bar{u}_{3}^{b}(0)$

This is a crucial part of this paper. It is derived from the following general lemma:

Lemma 2. Assume that $\varepsilon$ and $r^{\varepsilon}$ tend to zero, with $0<\varepsilon^{2} \ll r^{\varepsilon}$. Let $u^{b \varepsilon} \in\left(H^{1}\left(\Omega^{b}\right)\right)^{3}$ be such that:

$$
\begin{gathered}
u^{b \varepsilon}=0 \text { on } \Sigma^{b}, \\
\left\{e^{b \varepsilon}\left(u^{b \varepsilon}\right)\right\}_{\varepsilon} \text { is bounded in }\left(L^{2}\left(\Omega^{b}\right)\right)^{3 \times 3},
\end{gathered}
$$

with $e^{b \varepsilon}$ defined in (2.2). Then, up to a subsequence:

$$
u^{b \varepsilon} \rightarrow u^{b} \text { weakly in }\left(H^{1}\left(\Omega^{b}\right)\right)^{3},
$$

for some $u^{b} \in \mathcal{U}^{b}$ (in particular $u_{3}^{b} \in H_{0}^{2}\left(\omega^{b}\right)$ ). Moreover $u_{3}^{b \varepsilon}\left(r^{\varepsilon} ., 0\right)$ tends to $u_{3}^{b}(0)$ strongly in $L^{2}\left(\omega^{a}\right)$, for every $\omega^{a}$ such that $r^{\varepsilon} \omega^{a} \subset \omega^{b}$, for any $\varepsilon$.

Proof. The first part of the lemma is classical (see [5]). Let us prove the convergence of $u_{3}^{b \varepsilon}\left(r^{\varepsilon}\right.$.,0). We define $U^{\varepsilon}: \omega^{b} \rightarrow \mathbf{R}$ by:

$$
U^{\varepsilon}\left(x^{\prime}\right)=k \int_{-1}^{0} \int_{-1}^{0} \int_{t<x_{3}<t^{\prime}} u_{3}^{b \varepsilon}\left(x^{\prime}, x_{3}\right) \mathrm{d} x_{3} \mathrm{~d} t \mathrm{~d} t^{\prime}=k \int_{-1}^{0} \int_{-1}^{0} \rho\left(t, t^{\prime}\right) \int_{t}^{t^{\prime}} u_{3}^{b \varepsilon}\left(x^{\prime}, x_{3}\right) \mathrm{d} x_{3} \mathrm{~d} t \mathrm{~d} t^{\prime}
$$

with $\rho\left(t, t^{\prime}\right)=1$ if $t<t^{\prime}, 0$ otherwise, and with $k$ chosen so that:

$$
k \int_{-1}^{0} \int_{-1}^{0} \rho\left(t, t^{\prime}\right)\left(t^{\prime}-t\right) \mathrm{d} t \mathrm{~d} t^{\prime}=1 .
$$


Moreover we define $e_{\alpha}^{\varepsilon}: \Omega^{b} \rightarrow \mathbf{R}, E_{\alpha}^{\varepsilon}$ and $D_{\alpha}^{\varepsilon}: \omega^{b} \rightarrow \mathbf{R}$ by:

$$
\begin{gathered}
e_{\alpha}^{\varepsilon}=2 e_{\alpha 3}\left(u^{b \varepsilon}\right)=\frac{\partial u_{\alpha}^{b \varepsilon}}{\partial x_{3}}+\frac{\partial u_{3}^{b \varepsilon}}{\partial x_{\alpha}} \\
E_{\alpha}^{\varepsilon}\left(x^{\prime}\right)=k \int_{-1}^{0} \int_{-1}^{0} \rho\left(t, t^{\prime}\right) \int_{t}^{t^{\prime}} e_{\alpha}^{\varepsilon}\left(x^{\prime}, x_{3}\right) \mathrm{d} x_{3} \mathrm{~d} t \mathrm{~d} t^{\prime} \\
D_{\alpha}^{\varepsilon}\left(x^{\prime}\right)=k \int_{-1}^{0} \int_{-1}^{0} \rho\left(t, t^{\prime}\right) \int_{t}^{t^{\prime}} \frac{\partial u_{\alpha}^{b \varepsilon}}{\partial x_{3}}\left(x^{\prime}, x_{3}\right) \mathrm{d} x_{3} \mathrm{~d} t \mathrm{~d} t^{\prime}=k \int_{-1}^{0} \int_{-1}^{0} \rho\left(t, t^{\prime}\right)\left(u_{\alpha}^{b \varepsilon}\left(x^{\prime}, t^{\prime}\right)-u_{\alpha}^{b \varepsilon}\left(x^{\prime}, t\right)\right) \mathrm{d} t \mathrm{~d} t^{\prime} .
\end{gathered}
$$

It is clear that:

$$
\nabla U^{\varepsilon}=E^{\varepsilon}-D^{\varepsilon}
$$

Still denoting by $C$ various constants that do not depend on $\varepsilon$, we have from Cauchy-Schwarz inequality:

$$
\left|E_{\alpha}^{\varepsilon}\left(x^{\prime}\right)\right|^{2} \leq C \int_{-1}^{0}\left|e_{\alpha}^{\varepsilon}\left(x^{\prime}, x_{3}\right)\right|^{2} \mathrm{~d} x_{3}
$$

which gives, by definition of $e_{\alpha}^{\varepsilon}$ and by (5.1):

$$
\left\|E_{\alpha}^{\varepsilon}\right\|_{L^{2}\left(\omega^{b}\right)} \leq C\left\|e_{\alpha}^{\varepsilon}\right\|_{L^{2}\left(\Omega^{b}\right)}=C\left\|e_{\alpha 3}\left(u^{b \varepsilon}\right)\right\|_{L^{2}\left(\Omega^{b}\right)} \leq C \varepsilon .
$$

From (5.7), Cauchy-Schwarz inequality and the boundedness of $u_{\alpha}^{b \varepsilon}$ in $H_{0}^{1}\left(\Omega^{b}\right)$, we have:

$$
\left\|D_{\alpha}^{\varepsilon}\right\|_{H_{0}^{1}\left(\omega^{b}\right)} \leq C\left\|u_{\alpha}^{b \varepsilon}\right\|_{H_{0}^{1}\left(\Omega^{b}\right)} \leq C .
$$

From (5.8), we get the following decomposition:

$$
U^{\varepsilon}=\hat{U}^{\varepsilon}+\tilde{U}^{\varepsilon},
$$

with $\hat{U}^{\varepsilon}, \tilde{U}^{\varepsilon}$ the respective solutions in $H_{0}^{1}\left(\omega^{b}\right)$ of:

$$
-\Delta \hat{U}^{\varepsilon}=-\operatorname{div} E^{\varepsilon} \quad \text { and } \quad-\Delta \tilde{U}^{\varepsilon}=\operatorname{div} D^{\varepsilon} \quad \text { in } \omega^{b},
$$

and from (5.9), (5.10):

$$
\begin{gathered}
\left\|\nabla \hat{U}^{\varepsilon}\right\|_{\left(L^{2}\left(\omega^{b}\right)\right)^{2}} \leq\left\|E^{\varepsilon}\right\|_{\left(L^{2}\left(\omega^{b}\right)\right)^{2}} \leq C \varepsilon, \\
\hat{U}^{\varepsilon} \rightarrow 0 \text { in } H_{0}^{1}\left(\omega^{b}\right), \\
\left\|\tilde{U}^{\varepsilon}\right\|_{H^{2}\left(\omega^{b}\right)} \leq C\left\|\operatorname{div} D^{\varepsilon}\right\|_{L^{2}\left(\omega^{b}\right)} \leq C .
\end{gathered}
$$

But, using (5.2) and (5.4), it is easy to prove that:

$$
U^{\varepsilon} \rightarrow u_{3}^{b}=u_{3}^{b}\left(x^{\prime}\right) \text { weakly in } L^{2}\left(\omega^{b}\right),
$$

which gives, by virtue of (5.12), (5.13):

$$
\tilde{U}^{\varepsilon}=U^{\varepsilon}-\hat{U}^{\varepsilon} \rightarrow u_{3}^{b} \text { weakly in } H^{2}\left(\omega^{b}\right) .
$$


Then, as the embedding $H^{2}\left(\omega^{b}\right) \subset \mathcal{C}^{0}\left(\overline{\omega^{b}}\right)$ is compact, for $\omega^{b}$ bidimensional, we get that:

$$
\tilde{U}^{\varepsilon} \rightarrow u_{3}^{b} \text { in } \mathcal{C}^{0}\left(\overline{\omega^{b}}\right) .
$$

This is enough to prove that $u_{3}^{b \varepsilon}\left(r^{\varepsilon} ., 0\right)$ tends to $u_{3}^{b}(0)$ strongly in $L^{2}\left(\omega^{a}\right)$.

Actually we have, for a.e. $x^{\prime}$ in $\omega^{a}$,

$$
\left\{\begin{array}{l}
u_{3}^{b \varepsilon}\left(r^{\varepsilon} x^{\prime}, 0\right)-u_{3}^{b}(0) \\
=\left[u_{3}^{b \varepsilon}\left(r^{\varepsilon} x^{\prime}, 0\right)-U^{\varepsilon}\left(r^{\varepsilon} x^{\prime}\right)\right]+\left[U^{\varepsilon}\left(r^{\varepsilon} x^{\prime}\right)-\tilde{U}^{\varepsilon}\left(r^{\varepsilon} x^{\prime}\right)\right]+\left[\tilde{U}^{\varepsilon}\left(r^{\varepsilon} x^{\prime}\right)-u_{3}^{b}\left(r^{\varepsilon} x^{\prime}\right)\right]+\left[u_{3}^{b}\left(r^{\varepsilon} x^{\prime}\right)-u_{3}^{b}(0)\right] .
\end{array}\right.
$$

We will show that each of the above brackets tends strongly to zero in $L^{2}\left(\omega^{a}\right)$.

As for the first bracket, we have:

$$
\int_{\omega^{a}}\left|u_{3}^{b \varepsilon}\left(r^{\varepsilon} x^{\prime}, 0\right)-U^{\varepsilon}\left(r^{\varepsilon} x^{\prime}\right)\right|^{2} \mathrm{~d} x^{\prime}=\frac{1}{\left(r^{\varepsilon}\right)^{2}} \int_{r^{\varepsilon} \omega^{a}}\left|u_{3}^{b \varepsilon}\left(x^{\prime}, 0\right)-U^{\varepsilon}\left(x^{\prime}\right)\right|^{2} \mathrm{~d} x^{\prime} .
$$

But, by using (5.4):

$$
\begin{aligned}
U^{\varepsilon}\left(x^{\prime}\right) & =k \int_{-1}^{0} \int_{-1}^{0} \rho\left(t, t^{\prime}\right) \int_{t}^{t^{\prime}}\left(u_{3}^{b \varepsilon}\left(x^{\prime}, 0\right)+\int_{0}^{x_{3}} \frac{\partial u_{3}^{b \varepsilon}}{\partial x_{3}}\left(x^{\prime}, y_{3}\right) \mathrm{d} y_{3}\right) \mathrm{d} x_{3} \mathrm{~d} t \mathrm{~d} t^{\prime} \\
& =u_{3}^{b \varepsilon}\left(x^{\prime}, 0\right)+k \int_{-1}^{0} \int_{-1}^{0} \rho\left(t, t^{\prime}\right) \int_{t}^{t^{\prime}} \int_{0}^{x_{3}} \frac{\partial u_{3}^{b \varepsilon}}{\partial x_{3}}\left(x^{\prime}, y_{3}\right) \mathrm{d} y_{3} \mathrm{~d} x_{3} \mathrm{~d} t \mathrm{~d} t^{\prime},
\end{aligned}
$$

so that:

$$
\left|U^{\varepsilon}\left(x^{\prime}\right)-u_{3}^{b \varepsilon}\left(x^{\prime}, 0\right)\right| \leq C \int_{-1}^{0}\left|\frac{\partial u_{3}^{b \varepsilon}}{\partial x_{3}}\left(x^{\prime}, y_{3}\right)\right| \mathrm{d} y_{3},
$$

which combined with (5.1) gives:

$$
\int_{r^{\varepsilon} \omega^{a}}\left|u_{3}^{b \varepsilon}\left(x^{\prime}, 0\right)-U^{\varepsilon}\left(x^{\prime}\right)\right|^{2} \mathrm{~d} x^{\prime} \leq C \int_{\Omega^{b}}\left|\frac{\partial u_{3}^{b \varepsilon}}{\partial x_{3}}\right|^{2} \mathrm{~d} x \leq C \varepsilon^{4} .
$$

Coming back to (5.16), it results that:

$$
\int_{\omega^{a}}\left|u_{3}^{b \varepsilon}\left(r^{\varepsilon} x^{\prime}, 0\right)-U^{\varepsilon}\left(r^{\varepsilon} x^{\prime}\right)\right|^{2} \mathrm{~d} x^{\prime} \leq C \frac{\varepsilon^{4}}{\left(r^{\varepsilon}\right)^{2}},
$$

which tends to zero, since we have assumed that $\varepsilon^{2} \ll r^{\varepsilon}$. Now we consider the second bracket in (5.15), that is $\hat{U}^{\varepsilon}\left(r^{\varepsilon} x^{\prime}\right)$, and we are going to prove that its $L^{2}$-norm tends to zero, again if $\varepsilon^{2} \ll r^{\varepsilon}$. In fact, from CauchySchwarz inequality, the continuity of the embedding $H_{0}^{1}\left(\omega^{b}\right) \subset L^{4}\left(\omega^{b}\right)$ (actually $L^{q}\left(\omega^{b}\right)$, for every finite $q$, in dimension 2) and from (5.11):

$$
\begin{aligned}
\int_{\omega^{a}}\left|\hat{U}^{\varepsilon}\left(r^{\varepsilon} x^{\prime}\right)\right|^{2} \mathrm{~d} x^{\prime} & =\frac{1}{\left(r^{\varepsilon}\right)^{2}} \int_{r^{\varepsilon} \omega^{a}}\left|\hat{U}^{\varepsilon}\left(x^{\prime}\right)\right|^{2} \mathrm{~d} x^{\prime} \leq \frac{1}{\left(r^{\varepsilon}\right)^{2}}\left(\int_{r^{\varepsilon} \omega^{a}}\left|\hat{U}^{\varepsilon}\left(x^{\prime}\right)\right|^{4} \mathrm{~d} x^{\prime}\right)^{\frac{1}{2}}\left|r^{\varepsilon} \omega^{a}\right|^{\frac{1}{2}} \\
& \leq C \frac{1}{r^{\varepsilon}}\left\|\hat{U}^{\varepsilon}\right\|_{L^{4}\left(\omega^{b}\right)}^{2} \leq C \frac{1}{r^{\varepsilon}}\left\|\hat{U}^{\varepsilon}\right\|_{H_{0}^{1}\left(\omega^{b}\right)}^{2} \leq C \frac{\varepsilon^{2}}{r^{\varepsilon}} .
\end{aligned}
$$

By virtue of (5.14), the third and the fourth brackets in (5.15) tend to zero in $L^{\infty}\left(\omega^{a}\right)$. This concludes the proof of Lemma 2. 
Application: If $q \neq 0$, we write the junction condition for $\bar{u}_{3}^{\varepsilon}$ as:

$$
\bar{u}_{3}^{a \varepsilon}\left(x^{\prime}, 0\right)=\bar{u}_{3}^{b \varepsilon}\left(r^{\varepsilon} x^{\prime}, 0\right), \quad \text { for a.e. } x^{\prime} \in \omega^{a} .
$$

The left-hand side tends to $\bar{u}_{3}^{a}\left(x^{\prime}, 0\right)$ in $L^{2}\left(\omega^{a}\right)$ while the right-hand side tends to $\bar{u}_{3}^{b}(0)$ in the same space by Lemma 2. It follows that $\bar{u}_{3}^{a}\left(x^{\prime}, 0\right)=\bar{u}_{3}^{b}(0)$ for a.e. $x^{\prime}$ in $\omega^{a}$. If $q=0$ the same proof applies to $\tilde{u}^{\varepsilon}=q^{\varepsilon} \bar{u}^{\varepsilon}$.

\subsection{Proof of $\bar{c}(0)=0$}

This proof also is crucial.

Lemma 3. Assume that $\varepsilon$ and $r^{\varepsilon}$ tend to zero, with $0<\varepsilon^{2} \ll r^{\varepsilon}$. Let $\left(u^{a \varepsilon}, u^{b \varepsilon}\right) \in\left(H^{1}\left(\Omega^{a}\right)\right)^{3} \times\left(H^{1}\left(\Omega^{b}\right)\right)^{3}$ be such that:

$$
\begin{gathered}
u_{\mid x_{3}=1}^{a \varepsilon}=0, \\
u_{\alpha}^{a \varepsilon}\left(x^{\prime}, 0\right)=\varepsilon r^{\varepsilon} u_{\alpha}^{b \varepsilon}\left(r^{\varepsilon} x^{\prime}, 0\right), \quad \text { a.e. } x^{\prime} \in \omega^{a}, \\
\left\{e^{a \varepsilon}\left(u^{a \varepsilon}\right)\right\}_{\varepsilon} \text { is bounded in }\left(L^{2}\left(\Omega^{a}\right)\right)^{3 \times 3}, \\
\left\{u_{\alpha}^{b \varepsilon}\right\}_{\varepsilon} \text { is bounded in } H^{1}\left(\Omega^{b}\right) .
\end{gathered}
$$

Let $c^{\varepsilon}$ be defined by:

$$
c^{\varepsilon}\left(x_{3}\right)=\frac{\int_{\omega^{a}}\left(x_{1} u_{2}^{a \varepsilon}\left(x^{\prime}, x_{3}\right)-x_{2} u_{1}^{a \varepsilon}\left(x^{\prime}, x_{3}\right)\right) \mathrm{d} x^{\prime}}{r^{\varepsilon} \int_{\omega^{a}}\left(x_{1}^{2}+x_{2}^{2}\right) \mathrm{d} x^{\prime}} .
$$

Then $c^{\varepsilon}$ tends to $c$ in $L^{2}(0,1)$, where $c$ belongs to $H_{0}^{1}(0,1)$.

Proof. For $\alpha=1,2$, we define $x_{\alpha}^{R}$ by

and we set:

$$
x_{1}^{R}=-x_{2}, \quad x_{2}^{R}=x_{1},
$$

$$
v^{a \varepsilon}=\frac{u^{a \varepsilon}}{r^{\varepsilon}}, \quad e_{\alpha}^{\varepsilon}=2 e_{\alpha 3}\left(v^{a \varepsilon}\right)=2 e_{\alpha 3}^{a \varepsilon}\left(u^{a \varepsilon}\right),
$$

(this notation should not be confused with the notation $e_{\alpha}^{\varepsilon}$ appearing in (5.5)),

$$
m_{\alpha}^{\varepsilon}=\frac{1}{\left|\omega^{a}\right|} \int_{\omega^{a}} v_{\alpha}^{a \varepsilon} \mathrm{d} x^{\prime}, \quad \rho_{\alpha}^{\varepsilon}=\frac{1}{r^{\varepsilon}}\left[v_{\alpha}^{a \varepsilon}-c^{\varepsilon} x_{\alpha}^{R}-m_{\alpha}^{\varepsilon}\right],
$$

with $c^{\varepsilon}$ given by (5.21).

We begin by proving two a priori estimates. Due to (5.19), we have:

$$
\left\|e^{\varepsilon}\right\|_{\left(L^{2}\left(\Omega^{a}\right)\right)^{2}} \leq C .
$$

As for $\rho^{\varepsilon}$, it follows from (1.1) that $\rho_{\alpha}^{\varepsilon}\left(., x_{3}\right)$ has mean-value zero on $\omega^{a}$, for every $x_{3}$ and, as $e_{\alpha \beta}\left(\rho^{\varepsilon}\right)=$ $\left(1 / r^{\varepsilon}\right) e_{\alpha \beta}\left(v^{a \varepsilon}\right)$, we get from the Poincaré-Wirtinger inequality for elasticity:

$$
\left\|\rho^{\varepsilon}\right\|_{\left(L^{2}\left(\Omega^{a}\right)\right)^{2}}^{2} \leq C \sum_{\alpha \beta}\left\|e_{\alpha \beta}\left(\rho^{\varepsilon}\right)\right\|_{L^{2}\left(\Omega^{a}\right)}^{2}=\frac{C}{\left(r^{\varepsilon}\right)^{2}} \sum_{\alpha \beta}\left\|e_{\alpha \beta}\left(v^{a \varepsilon}\right)\right\|_{L^{2}\left(\Omega^{a}\right)}^{2}=C \sum_{\alpha \beta}\left\|e_{\alpha \beta}^{a \varepsilon}\left(u^{a \varepsilon}\right)\right\|_{L^{2}\left(\Omega^{a}\right)}^{2},
$$

which gives, with (5.19):

$$
\left\|\rho^{\varepsilon}\right\|_{\left(L^{2}\left(\Omega^{a}\right)\right)^{2}} \leq C
$$


Now we prove that one can derive a single equation, of the form $c^{\varepsilon}=K^{\varepsilon}-r^{\varepsilon} R^{\varepsilon}$, from the system of two equations $c^{\varepsilon} x_{\alpha}^{R}+m_{\alpha}^{\varepsilon}=v_{\alpha}^{a \varepsilon}-r^{\varepsilon} \rho_{\alpha}^{\varepsilon}$. This is a cleaver argument appearing in [28], see also Section 8.1. Indeed we get by differentiating the previous system with respect to $x_{3}$ :

$$
\frac{\mathrm{d} c^{\varepsilon}}{\mathrm{d} x_{3}} x_{\alpha}^{R}+\frac{\mathrm{d} m_{\alpha}^{\varepsilon}}{\mathrm{d} x_{3}}+\frac{\partial v_{3}^{a \varepsilon}}{\partial x_{\alpha}}=e_{\alpha}^{\varepsilon}-r^{\varepsilon} \frac{\partial \rho_{\alpha}^{\varepsilon}}{\partial x_{3}}, \quad \forall \alpha=1,2 .
$$

After multiplying (5.24) by a test function $\varphi_{\alpha} \in \mathcal{D}\left(\omega^{a}\right)$, summing over $\alpha$ and integrating over $\omega^{a}$, we have:

$$
\left\{\begin{array}{l}
\frac{\mathrm{d} c^{\varepsilon}}{\mathrm{d} x_{3}} \int_{\omega^{a}} \sum_{\alpha} \varphi_{\alpha} x_{\alpha}^{R} \mathrm{~d} x^{\prime}+\sum_{\alpha} \frac{\mathrm{d} m_{\alpha}^{\varepsilon}}{\mathrm{d} x_{3}} \int_{\omega^{a}} \varphi_{\alpha} \mathrm{d} x^{\prime}-\int_{\omega^{a}} v_{3}^{a \varepsilon} \operatorname{div} \varphi \mathrm{d} x^{\prime} \\
=\int_{\omega^{a}} \sum_{\alpha} e_{\alpha}^{\varepsilon} \varphi_{\alpha} \mathrm{d} x^{\prime}-r^{\varepsilon} \frac{\mathrm{d}}{\mathrm{d} x_{3}} \int_{\omega^{a}} \sum_{\alpha} \rho_{\alpha}^{\varepsilon} \varphi_{\alpha} \mathrm{d} x^{\prime} .
\end{array}\right.
$$

We choose the test function $\varphi_{\alpha}$ so that:

$$
\begin{gathered}
\int_{\omega^{a}} \sum_{\alpha} \varphi_{\alpha} x_{\alpha}^{R} \mathrm{~d} x^{\prime}=1, \\
\int_{\omega^{a}} \varphi_{\alpha} \mathrm{d} x^{\prime}=0, \quad \forall \alpha=1,2, \\
\operatorname{div} \varphi=0 .
\end{gathered}
$$

It is easy to check that such test function does exist: take e.g.

$$
\varphi_{1}=\frac{\partial \phi}{\partial x_{2}}, \quad \varphi_{2}=-\frac{\partial \phi}{\partial x_{1}}, \quad \text { with } \quad \phi \in \mathcal{D}\left(\omega^{a}\right), \quad \int_{\omega^{a}} \phi \mathrm{d} x^{\prime}=\frac{1}{2} .
$$

Now we set (this notation should not be confused with the notation $E^{\varepsilon}$ appearing in (5.6)):

$$
E^{\varepsilon}=\int_{\omega^{a}} e^{\varepsilon} \cdot \varphi \mathrm{d} x^{\prime}, \quad K^{\varepsilon}=-\int_{x_{3}}^{1} E^{\varepsilon}\left(y_{3}\right) \mathrm{d} y_{3}, \quad R^{\varepsilon}=\int_{\omega^{a}} \rho^{\varepsilon} \cdot \varphi \mathrm{d} x^{\prime},
$$

where . denotes the scalar product in $\mathbf{R}^{2}$. Then (5.25) reads as:

$$
\frac{\mathrm{d} c^{\varepsilon}}{\mathrm{d} x_{3}}=\frac{\mathrm{d} K^{\varepsilon}}{\mathrm{d} x_{3}}-r^{\varepsilon} \frac{\mathrm{d} R^{\varepsilon}}{\mathrm{d} x_{3}}
$$

which gives by integration:

$$
c^{\varepsilon}=K^{\varepsilon}-r^{\varepsilon} R^{\varepsilon}
$$

since $c^{\varepsilon}(1)=K^{\varepsilon}(1)=0$ and since also $R^{\varepsilon}(1)=0$, because $\rho^{\varepsilon}(1)=0$.

Now we pass to the limit in (5.29). Due to (5.22) and (5.23), $E^{\varepsilon}$ and $R^{\varepsilon}$ are bounded in $L^{2}(0,1)$. Moreover it follows that $K^{\varepsilon}$ is bounded in $H^{1}(0,1)$, since by Poincaré inequality one has:

$$
\left\|K^{\varepsilon}\right\|_{H^{1}(0,1)}^{2} \leq C \int_{0}^{1}\left|\frac{\mathrm{d} K^{\varepsilon}}{\mathrm{d} x_{3}}\right|^{2} \mathrm{~d} x_{3}=C \int_{0}^{1}\left|E^{\varepsilon}\right|^{2} \mathrm{~d} x_{3} \leq C .
$$

Then we deduce that there exists $c$ in $H^{1}(0,1)$, with $c(1)=0$, such that:

$$
K^{\varepsilon} \rightarrow c \text { weakly in } H^{1}(0,1) \text {, hence } K^{\varepsilon} \rightarrow c \text { strongly in } \mathcal{C}^{0}(0,1) .
$$

As $r^{\varepsilon} R^{\varepsilon}$ tends to zero strongly in $L^{2}(0,1)$, it follows from (5.29) and the above that $c^{\varepsilon}$ tends to $c$ strongly in $L^{2}(0,1)$. 
It remains to prove that $c$ vanishes at the origin. For this, we notice that $K^{\varepsilon}(0) \rightarrow c(0)$. But one has also another expression for $K^{\varepsilon}$. Actually, from (5.29), (5.26), (5.27) and by the definition of $\rho_{\alpha}^{\varepsilon}$ :

$$
\begin{aligned}
K^{\varepsilon}=r^{\varepsilon} R^{\varepsilon}+c^{\varepsilon} & =r^{\varepsilon} \int_{\omega^{a}} \rho^{\varepsilon} \cdot \varphi \mathrm{d} x^{\prime}+c^{\varepsilon} \int_{\omega^{a}} \sum_{\alpha} \varphi_{\alpha} x_{\alpha}^{R} \mathrm{~d} x^{\prime}+\sum_{\alpha} m_{\alpha}^{\varepsilon} \int_{\omega^{a}} \sum_{\alpha} \varphi_{\alpha} \mathrm{d} x^{\prime} \\
& =\sum_{\alpha} \int_{\omega^{a}}\left(r^{\varepsilon} \rho_{\alpha}^{\varepsilon}+c^{\varepsilon} x_{\alpha}^{R}+m_{\alpha}^{\varepsilon}\right) \varphi_{\alpha} \mathrm{d} x^{\prime}=\sum_{\alpha} \int_{\omega^{a}} v_{\alpha}^{a \varepsilon} \varphi_{\alpha} \mathrm{d} x^{\prime}
\end{aligned}
$$

that is:

$$
K^{\varepsilon}=\sum_{\alpha} \int_{\omega^{a}} v_{\alpha}^{a \varepsilon} \varphi_{\alpha} \mathrm{d} x^{\prime}=\sum_{\alpha} \int_{\omega^{a}} \frac{1}{r^{\varepsilon}} u_{\alpha}^{a \varepsilon} \varphi_{\alpha} \mathrm{d} x^{\prime},
$$

and in particular, due to the boundary condition (5.18):

$$
K^{\varepsilon}(0)=\varepsilon \sum_{\alpha} \int_{\omega^{a}} u_{\alpha}^{b \varepsilon}\left(r^{\varepsilon} x^{\prime}, 0\right) \varphi_{\alpha} \mathrm{d} x^{\prime} .
$$

Hence, by using Hölder inequality, the continuity of the embedding of $H^{\frac{1}{2}}\left(\omega^{b}\right)$ in $L^{4}\left(\omega^{b}\right)$ (in dimension 2), the continuity of the trace mapping from $H^{1}\left(\Omega^{b}\right)$ to $H^{\frac{1}{2}}\left(\omega^{b}\right)$ and $(5.20)$, we get:

$$
\begin{aligned}
\left|K^{\varepsilon}(0)\right| & \leq C \varepsilon \sum_{\alpha} \int_{\omega^{a}}\left|u_{\alpha}^{b \varepsilon}\left(r^{\varepsilon} x^{\prime}, 0\right)\right| \mathrm{d} x^{\prime}=C \frac{\varepsilon}{\left(r^{\varepsilon}\right)^{2}} \sum_{\alpha} \int_{r^{\varepsilon} \omega^{a}}\left|u_{\alpha}^{b \varepsilon}\left(x^{\prime}, 0\right)\right| \mathrm{d} x^{\prime} \\
& \leq C \frac{\varepsilon}{\left(r^{\varepsilon}\right)^{2}} \sum_{\alpha}\left[\int_{r^{\varepsilon} \omega^{a}}\left|u_{\alpha}^{b \varepsilon}\left(x^{\prime}, 0\right)\right|^{4} \mathrm{~d} x^{\prime}\right]^{\frac{1}{4}}\left|r^{\varepsilon} \omega^{a}\right|^{\frac{3}{4}}=C \varepsilon\left(r^{\varepsilon}\right)^{-\frac{1}{2}} \sum_{\alpha}\left[\int_{r^{\varepsilon} \omega^{a}}\left|u_{\alpha}^{b \varepsilon}\left(x^{\prime}, 0\right)\right|^{4} \mathrm{~d} x^{\prime}\right]^{\frac{1}{4}} \\
& \leq C \varepsilon\left(r^{\varepsilon}\right)^{-\frac{1}{2}} \sum_{\alpha}\left\|u_{\alpha}^{b \varepsilon}(., 0)\right\|_{L^{4}\left(\omega^{b}\right)} \leq C \varepsilon\left(r^{\varepsilon}\right)^{-\frac{1}{2}} \sum_{\alpha}\left\|u_{\alpha}^{b \varepsilon}(., 0)\right\|_{H^{\frac{1}{2}}\left(\Omega^{b}\right)} \leq C \varepsilon\left(r^{\varepsilon}\right)^{-\frac{1}{2}},
\end{aligned}
$$

which tends to zero, since $0<\varepsilon^{2} \ll r^{\varepsilon}$. As we have proved that $K^{\varepsilon}(0) \rightarrow c(0)$, we conclude that $c(0)=0$.

\section{The Use of CONVEnient TEST FunCtions}

This is the third crucial part of the paper, at least in the case $0<q<+\infty$.

\subsection{The case $q=+\infty$}

Observe that $\mathcal{Z}_{\infty}=\left\{z^{a} \in \mathcal{Z}^{a}, \zeta^{a}\left(=\zeta^{a}\left(u^{a}\right)\right) \in H_{0}^{1}(0,1)\right\}$. Let $u^{a} \in \mathcal{U}^{a}$, with $\zeta^{a} \in H_{0}^{1}(0,1)$ and let $\left(v^{a}, w^{a}\right)$ be such that:

$$
\begin{aligned}
& v_{1}^{a}=-c x_{2} \text { and } v_{2}^{a}=c x_{1} \text { with } c \in H_{0}^{1}(0,1), v_{3}^{a} \in \mathcal{C}^{1}\left(\overline{\Omega^{a}}\right), v_{3 \mid x_{3}=0}^{a}=0, \\
& w_{\alpha}^{a} \in \mathcal{C}^{1}\left(\overline{\Omega^{a}}\right), w_{\alpha \mid x_{3}=0}^{a}=0, w_{3}^{a}=0 .
\end{aligned}
$$

In other words, $v^{a}$ and $w^{a}$ satisfy all the conditions given in the definitions of $\mathcal{V}^{a}$ and $\mathcal{W}^{a}$, but the integral ones; moreover $v_{3}^{a}$ and $w_{\alpha}^{a}$ belong to the space $\mathcal{R}$ defined by:

$$
\mathcal{R}=\left\{v \in \mathcal{C}^{1}\left(\overline{\Omega^{a}}\right), v_{\mid x_{3}=0}=0\right\} .
$$


Let $u^{a \varepsilon}=u^{a}+r^{\varepsilon} v^{a}+\left(r^{\varepsilon}\right)^{2} w^{a}$. Then it is easy to check that $u=\left(u^{a \varepsilon}, 0\right)$ is in $\mathcal{Y}^{\varepsilon}$. Taking it as test function in the variational equation of problem (1.2), we get:

$$
\int_{\Omega^{a}}\left[A^{a} \bar{e}^{a \varepsilon}, e^{a \varepsilon}\left(u^{a \varepsilon}\right)\right] \mathrm{d} x=\int_{\Omega^{a}} f^{a \varepsilon} \cdot u^{a \varepsilon} \mathrm{d} x+\int_{\Omega^{a}}\left[g^{a \varepsilon}, e^{a \varepsilon}\left(u^{a \varepsilon}\right)\right] \mathrm{d} x+\int_{\Sigma^{a}} h^{a \varepsilon} \cdot u^{a \varepsilon} \mathrm{d} \sigma .
$$

But we have, since $e_{\alpha \beta}\left(u^{a}\right)=e_{\alpha 3}\left(u^{a}\right)=e_{\alpha \beta}\left(v^{a}\right)=e_{33}\left(w^{a}\right)=0$ :

$$
e^{a \varepsilon}\left(u^{a \varepsilon}\right)=\left(\begin{array}{cc}
e_{\alpha \beta}\left(w^{a}\right) & e_{\alpha 3}\left(v^{a}\right) \\
e_{3 \alpha}\left(v^{a}\right) & e_{33}\left(u^{a}\right)
\end{array}\right)+r^{\varepsilon}\left(\begin{array}{cc}
0 & e_{\alpha 3}\left(w^{a}\right) \\
e_{3 \alpha}\left(w^{a}\right) & e_{33}\left(v^{a}\right)
\end{array}\right),
$$

so that $e^{a \varepsilon}\left(u^{a \varepsilon}\right)$ tends to $e^{a}\left(z^{a}\right)=e^{a}\left(u^{a}, v^{a}, w^{a}\right)$ (strongly) in $\left(L^{2}\left(\Omega^{a}\right)\right)^{3 \times 3}$. Moreover $u^{a \varepsilon}$ tends to $u^{a}$ (strongly) in $\left(H^{1}\left(\Omega^{a}\right)\right)^{3}$ and we have seen in (4.5) that $\bar{e}^{a \varepsilon}$ tends to $e^{a}\left(\bar{z}^{a}\right)$ weakly in $\left(L^{2}\left(\Omega^{a}\right)\right)^{3 \times 3}$. By passing to the limit in (6.3), using (2.7), (2.9), (2.11), it follows that:

$$
\int_{\Omega^{a}}\left[A^{a} e^{a}\left(\bar{z}^{a}\right), e^{a}\left(z^{a}\right)\right] \mathrm{d} x=\int_{\Omega^{a}} f^{a} \cdot u^{a} \mathrm{~d} x+\int_{\Omega^{a}}\left[g^{a}, e^{a}\left(z^{a}\right)\right] \mathrm{d} x+\int_{\Sigma^{a}} h^{a} \cdot u^{a} \mathrm{~d} \sigma,
$$

which is the variational equation of (2.18). It holds also true, by density and continuity, for every $\left(v^{a}, w^{a}\right)$ such that:

$$
\begin{aligned}
& v_{1}^{a}=-c x_{2} \text { and } v_{2}^{a}=c x_{1} \text { with } c \in H_{0}^{1}(0,1), \quad v_{3}^{a} \in L^{2}\left(0,1 ; H^{1}\left(\omega^{a}\right)\right), \\
& w_{\alpha}^{a} \in L^{2}\left(0,1 ; H^{1}\left(\omega^{a}\right)\right), \quad w_{3}^{a}=0,
\end{aligned}
$$

i.e. for every $\left(v^{a}, w^{a}\right)$ satisfying the conditions given in the definitions of $\mathcal{V}^{a}$ and $\mathcal{W}^{a}$, but the integral ones (note that $\mathcal{R}$ defined in $(6.2)$ is dense in $L^{2}\left(0,1 ; H^{1}\left(\omega^{a}\right)\right)$ ). In particular (6.4) is also true for any $z^{a} \in \mathcal{Z}_{\infty}$. This means that $\bar{z}^{a}$ solves the variational problem (2.18).

\subsection{The case $q=0$}

We have seen that $\tilde{u}^{\varepsilon}=q^{\varepsilon} \bar{u}^{\varepsilon}=q^{\varepsilon}\left(\bar{u}^{a \varepsilon}, \bar{u}^{b \varepsilon}\right)$ solves (4.3) and that (see (4.8)) $\tilde{u}^{a \varepsilon}=q^{\varepsilon} \bar{u}^{a \varepsilon}$ tends to $\bar{u}^{a}=0$ in $\left(H^{1}\left(\Omega^{a}\right)\right)^{3}, \tilde{e}^{b \varepsilon}=q^{\varepsilon} \bar{e}^{b \varepsilon}$ tends to $\bar{e}^{b}=e^{b}\left(\bar{z}^{b}\right)$ weakly in $\left(L^{2}\left(\Omega^{b}\right)\right)^{3 \times 3}$, for some $\bar{z}^{b}$ in $\mathcal{Z}_{0}$ (in particular $\left.\bar{u}_{3}^{b}(0)=0\right)$.

Let $B$ be some given small ball, with center zero, in $\omega^{b}$. Let $z^{b}=\left(u^{b}, v^{b}, w^{b}\right)$ be such that:

$$
\left\{\begin{array}{l}
u^{b} \in \mathcal{U}^{b}, \quad \zeta_{\alpha}^{b}\left(=\zeta_{\alpha}^{b}\left(u^{b}\right)\right)=u_{3}^{b}=0 \text { in } B, \\
v_{\alpha}^{b} \in \mathcal{C}^{1}\left(\overline{\Omega^{b}}\right), \quad v_{\alpha}^{b}=0 \text { in } B \times\{0\}, \quad v_{3}^{b}=0, \\
w_{\alpha}^{b}=0, \quad w_{3}^{b} \in \mathcal{C}^{1}\left(\overline{\Omega^{b}}\right), \quad w_{3}^{b}=0 \text { in } B \times\{0\} .
\end{array}\right.
$$

In other words, $z^{b}$ satisfies all the conditions given in the definition of $\mathcal{Z}_{0}$, except the integral ones; moreover $\zeta_{\alpha}^{b}$ and $u_{3}^{b}$ vanish in $B, v_{\alpha}^{b}$ and $w_{3}^{b}$ belong to $\mathcal{C}^{1}\left(\overline{\Omega^{b}}\right)$ and vanish in $B \times\{0\}$. Let $u^{b \varepsilon}=u^{b}+\varepsilon v^{b}+(\varepsilon)^{2} w^{b}$. Then it is easy to check that $u=\left(0, u^{b \varepsilon}\right)$ is in $\mathcal{Y}^{\varepsilon}$, for $\varepsilon$ small enough. Taking it as test function in the variational equation of problem (1.2), we get:

$$
\int_{\Omega^{b}}\left[A^{b} \tilde{e}^{b \varepsilon}, e^{b \varepsilon}\left(u^{b \varepsilon}\right)\right] \mathrm{d} x=\int_{\Omega^{b}} f^{b \varepsilon} \cdot u^{b \varepsilon} \mathrm{d} x+\int_{\Omega^{b}}\left[g^{b \varepsilon}, e^{b \varepsilon}\left(u^{b \varepsilon}\right)\right] \mathrm{d} x+\int_{\omega^{b}}\left(h_{+}^{b \varepsilon} \cdot u_{\mid x_{3}=0}^{b \varepsilon}+h_{-}^{b \varepsilon} \cdot u_{\mid x_{3}=-1}^{b \varepsilon}\right) \mathrm{d} x^{\prime} .
$$

But we have, since $e_{\alpha 3}\left(u^{b}\right)=e_{33}\left(u^{b}\right)=e_{33}\left(v^{b}\right)=e_{\alpha \beta}\left(w^{b}\right)=0$ :

$$
e^{b \varepsilon}\left(u^{b \varepsilon}\right)=\left(\begin{array}{cc}
e_{\alpha \beta}\left(u^{b}\right) & e_{\alpha 3}\left(v^{b}\right) \\
e_{3 \alpha}\left(v^{b}\right) & e_{33}\left(w^{b}\right)
\end{array}\right)+\varepsilon\left(\begin{array}{cc}
e_{\alpha \beta}\left(v^{b}\right) & e_{\alpha 3}\left(w^{b}\right) \\
e_{3 \alpha}\left(w^{b}\right) & 0
\end{array}\right),
$$


so that $e^{b \varepsilon}\left(u^{b \varepsilon}\right)$ tends to $e^{b}\left(z^{b}\right)$ (strongly) in $\left(L^{2}\left(\Omega^{b}\right)\right)^{3 \times 3}$. Moreover $u^{b \varepsilon}$ tends to $u^{b}$ (strongly) in $\left(H^{1}\left(\Omega^{b}\right)\right)^{3}$ and we have seen that $\tilde{e}^{b \varepsilon}$ tends to $e^{b}\left(\bar{z}^{b}\right)$ weakly in $\left(L^{2}\left(\Omega^{b}\right)\right)^{3 \times 3}$. By passing to the limit in (6.6), using (2.8), (2.10), (2.12), it follows that:

$$
\int_{\Omega^{b}}\left[A^{b} e^{b}\left(\bar{z}^{b}\right), e^{b}\left(z^{b}\right)\right] \mathrm{d} x=\int_{\Omega^{b}} f^{b} \cdot u^{b} \mathrm{~d} x+\int_{\Omega^{b}}\left[g^{b}, e^{b}\left(z^{b}\right)\right] \mathrm{d} x+\int_{\omega^{b}}\left(h_{+}^{b} \cdot u_{\mid x_{3}=0}^{b}+h_{-}^{b} \cdot u_{\mid x_{3}=-1}^{b}\right) \mathrm{d} x^{\prime},
$$

for every $z^{b}=\left(u^{b}, v^{b}, w^{b}\right)$ having the regularity (6.5).

But the following density results are proved in the Appendix (Sect. 8.2). First, from Lemma 5, any $\zeta_{\alpha}^{b} \in H_{0}^{1}\left(\omega^{b}\right)$ can be approximated (in the strong topology of $H_{0}^{1}\left(\omega^{b}\right)$ ) by a sequence $\zeta_{\alpha}^{b n}$ with $\zeta_{\alpha}^{b n}=0$ in a ball $B^{n}$ of radius $r^{n}$ tending to zero. Moreover, from Lemma 6 , any $u_{3}^{b} \in H_{0}^{2}\left(\omega^{b}\right)$, with $u_{3}^{b}(0)=0$ can be approximated (in the weak topology of $\left.H_{0}^{2}\left(\omega^{b}\right)\right)$ by a sequence $u_{3}^{b n}$ that vanishes in $B^{n}$. Finally, from Lemma 7 , any $v_{\alpha}^{b}\left(\right.$ or $\left.w_{3}^{b}\right)$ in $L^{2}\left(\omega^{b} ; H^{1}(-1,0)\right)$ can be approximated (in the strong topology of $L^{2}\left(\omega^{b} ; H^{1}(-1,0)\right)$ ) by a sequence of functions $v_{\alpha}^{n b}$ (or $w_{3}^{n b}$ ) in $\mathcal{C}^{1}\left(\overline{\Omega^{b}}\right)$ that vanish in $B^{n} \times\{0\}$.

By continuity, it results that (6.7) holds true for any $\left(u^{b}, v^{b}, w^{b}\right)$ such that:

$$
\begin{aligned}
& u^{b} \in \mathcal{U}^{b}, \quad u_{3}^{b}(0)=0, \\
& v_{\alpha}^{b} \in L^{2}\left(\omega^{b} ; H^{1}(-1,0)\right), \quad v_{3}^{b}=0, \\
& w_{\alpha}^{b}=0, \quad w_{3}^{b} \in L^{2}\left(\omega^{b} ; H^{1}(-1,0)\right),
\end{aligned}
$$

i.e. for every $z^{b}$ satisfying the conditions given in the definition of $\mathcal{Z}_{0}$, but the integral ones. In particular (6.7) is also true for any $z^{b} \in \mathcal{Z}_{0}$. This means that $\bar{z}^{b}$ solves the variational problem (2.23).

\subsection{The case $0<q<+\infty$}

Let $z=\left(z^{a}, z^{b}\right)=\left(\left(u^{a}, v^{a}, w^{a}\right),\left(u^{b}, v^{b}, w^{b}\right)\right) \in\left(\mathcal{C}^{1}\left(\overline{\Omega^{a}}\right)\right)^{3} \times\left(\mathcal{C}^{1}\left(\overline{\Omega^{b}}\right)\right)^{3}$. We assume that $z$ satisfies all the conditions required in the definition of $Z$, except the integral ones, and we assume that it is "more regular". In particular $u_{3}^{a}\left(x^{\prime}, 0\right)=u_{3}^{b}(0)$, that is $\zeta^{a}(0)=u_{3}^{b}(0)$. The precise requirements are given by:

$$
\left\{\begin{array}{l}
u^{a} \in \mathcal{U}^{a}, \quad u_{\alpha}^{a} \in \mathcal{C}^{2}[0,1], \quad \zeta^{a} \in \mathcal{C}^{1}[0,1], \\
v_{1}^{a}=-c x_{2} \text { and } v_{2}^{a}=c x_{1} \quad \text { with } c \in \mathcal{C}^{1}[0,1], \quad c(0)=c(1)=0, \quad v_{3}^{a} \in \mathcal{C}^{1}\left(\overline{\Omega^{a}}\right), \quad v_{3 \mid x_{3}=0}^{a}=0, \\
w_{\alpha}^{a} \in \mathcal{C}^{1}\left(\overline{\Omega^{a}}\right), \quad w_{\alpha \mid x_{3}=0}^{a}=0, \quad w_{3}^{a}=0, \\
u^{b} \in \mathcal{U}^{b}, \quad \zeta_{\alpha}^{b} \in \mathcal{C}^{1}\left(\overline{\omega^{b}}\right) \cap H_{0}^{1}\left(\omega^{b}\right), \quad u_{3}^{b} \in \mathcal{C}^{1}\left(\overline{\omega^{b}}\right) \cap H_{0}^{2}\left(\omega^{b}\right), \\
v^{b} \in\left(\mathcal{C}^{1}\left(\overline{\Omega^{b}}\right)\right)^{2} \times\{0\}, \quad v_{\alpha}^{b}=0 \text { on } \Sigma^{b}, \\
w^{b} \in(\{0\})^{2} \times \mathcal{C}^{1}\left(\overline{\Omega^{b}}\right), \quad w_{3}^{b}=0 \text { on } \Sigma^{b}, \\
u_{3}^{b}(0)=\zeta^{a}(0) .
\end{array}\right.
$$

We are going to define a convenient test function $u^{\varepsilon}=\left(u^{a \varepsilon}, u^{b \varepsilon}\right)$ in $\mathcal{Y}^{\varepsilon}$.

- In $\Omega^{b}$, we choose:

$$
u^{b \varepsilon}=u^{b}+\varepsilon v^{b}+\varepsilon^{2} w^{b} .
$$

As the couple $u^{\varepsilon}=\left(u^{a \varepsilon}, u^{b \varepsilon}\right)$ needs to satisfy the transmission conditions (3.1), i.e.:

$$
u_{\alpha}^{a}\left(x^{\prime}, 0\right)=\varepsilon r^{\varepsilon} u_{\alpha}^{b}\left(r^{\varepsilon} x^{\prime}, 0\right) \text { and } u_{3}^{a}\left(x^{\prime}, 0\right)=u_{3}^{b}\left(r^{\varepsilon} x^{\prime}, 0\right), \quad \text { for a.e. } x^{\prime} \in \omega^{a},
$$


then, necessarily, $u^{a \varepsilon}\left(x^{\prime}, 0\right)$ is given by:

- In $\Omega^{a} \cap\left\{x_{3}>r^{\varepsilon}\right\}$, we choose

$$
\begin{gathered}
u_{\alpha}^{a \varepsilon}\left(x^{\prime}, 0\right)=\varepsilon r^{\varepsilon}\left(\zeta_{\alpha}^{b}\left(r^{\varepsilon} x^{\prime}\right)+\varepsilon v_{\alpha}^{b}\left(r^{\varepsilon} x^{\prime}, 0\right)\right), \\
u_{3}^{a \varepsilon}\left(x^{\prime}, 0\right)=u_{3}^{b}\left(r^{\varepsilon} x^{\prime}\right)+\varepsilon^{2} w_{3}^{b}\left(r^{\varepsilon} x^{\prime}, 0\right) .
\end{gathered}
$$

$$
u^{a \varepsilon}=u^{a}+r^{\varepsilon} v^{a}+\left(r^{\varepsilon}\right)^{2} w^{a} .
$$

- In $\Omega^{a} \cap\left\{0<x_{3}<r^{\varepsilon}\right\}, u^{a \varepsilon}$ is obtained by linear interpolation between $u^{a \varepsilon}\left(x^{\prime}, 0\right)$ and $u^{a \varepsilon}\left(x^{\prime}, r^{\varepsilon}\right)$ :

$$
u^{a \varepsilon}\left(x^{\prime}, x_{3}\right)=\frac{x_{3}}{r^{\varepsilon}}\left(u^{a}\left(x^{\prime}, r^{\varepsilon}\right)+r^{\varepsilon} v^{a}\left(x^{\prime}, r^{\varepsilon}\right)+\left(r^{\varepsilon}\right)^{2} w^{a}\left(x^{\prime}, r^{\varepsilon}\right)\right)+\left(1-\frac{x_{3}}{r^{\varepsilon}}\right) u^{a \varepsilon}\left(x^{\prime}, 0\right),
$$

that is (see above):

$$
\begin{gathered}
u_{\alpha}^{a \varepsilon}\left(x^{\prime}, x_{3}\right)=\frac{x_{3}}{r^{\varepsilon}}\left(u_{\alpha}^{a}\left(r^{\varepsilon}\right)+r^{\varepsilon} v_{\alpha}^{a}\left(x^{\prime}, r^{\varepsilon}\right)+\left(r^{\varepsilon}\right)^{2} w_{\alpha}^{a}\left(x^{\prime}, r^{\varepsilon}\right)\right)+\left(1-\frac{x_{3}}{r^{\varepsilon}}\right) \varepsilon r^{\varepsilon}\left(\zeta_{\alpha}^{b}\left(r^{\varepsilon} x^{\prime}\right)+\varepsilon v_{\alpha}^{b}\left(r^{\varepsilon} x^{\prime}, 0\right)\right) \\
u_{3}^{a \varepsilon}\left(x^{\prime}, x_{3}\right)=\frac{x_{3}}{r^{\varepsilon}}\left(u_{3}^{a}\left(x^{\prime}, r^{\varepsilon}\right)+r^{\varepsilon} v_{3}^{a}\left(x^{\prime}, r^{\varepsilon}\right)\right)+\left(1-\frac{x_{3}}{r^{\varepsilon}}\right)\left(u_{3}^{b}\left(r^{\varepsilon} x^{\prime}\right)+\varepsilon^{2} w_{3}^{b}\left(r^{\varepsilon} x^{\prime}, 0\right)\right)
\end{gathered}
$$

for $0<x_{3}<r^{\varepsilon}$. Taking $u^{\varepsilon}=\left(u^{a \varepsilon}, u^{b \varepsilon}\right)$ as test function in the variational equation of problem (2.3), we get:

$$
\left\{\begin{array}{l}
\int_{\Omega^{a}}\left[A^{a} \bar{e}^{a \varepsilon}, e^{a \varepsilon}\left(u^{a \varepsilon}\right)\right] \mathrm{d} x+q^{\varepsilon} \int_{\Omega^{b}}\left[A^{b} \bar{e}^{b \varepsilon}, e^{b \varepsilon}\left(u^{b \varepsilon}\right)\right] \mathrm{d} x \\
=\int_{\Omega^{a}} f^{a \varepsilon} \cdot u^{a \varepsilon} \mathrm{d} x+\int_{\Omega^{b}} f^{b \varepsilon} \cdot u^{b \varepsilon} \mathrm{d} x+\int_{\Omega^{a}}\left[g^{a \varepsilon}, e^{a \varepsilon}\left(u^{a \varepsilon}\right)\right] \mathrm{d} x+\int_{\Omega^{b}}\left[g^{b \varepsilon}, e^{b \varepsilon}\left(u^{b \varepsilon}\right)\right] \mathrm{d} x \\
+\int_{\Sigma^{a}} h^{a \varepsilon} \cdot u^{a \varepsilon} \mathrm{d} \sigma+\int_{\omega^{b}}\left(h_{+}^{b \varepsilon} \cdot u_{\mid x_{3}=0}^{b \varepsilon}+h_{-}^{b \varepsilon} \cdot u_{\mid x_{3}=-1}^{b \varepsilon}\right) \mathrm{d} x^{\prime} .
\end{array}\right.
$$

Passing to the limit in the integral terms in $\Omega^{b}$ is easy. As for the terms in $\Omega^{a} \cap\left\{x_{3}>r^{\varepsilon}\right\}$ and $\Sigma^{a} \cap\left\{x_{3}>r^{\varepsilon}\right\}$, we have from Lebesgue's theorem, with $u^{a \varepsilon}=u^{a}+r^{\varepsilon} v^{a}+\left(r^{\varepsilon}\right)^{2} w^{a}$ and $\chi^{\varepsilon}$ the characteristic function of $\left\{x_{3}>r^{\varepsilon}\right\}$ :

$$
\begin{aligned}
& \chi^{\varepsilon} e^{a \varepsilon}\left(u^{a \varepsilon}\right) \rightarrow e^{a}\left(z^{a}\right) \text { strongly in }\left(L^{2}\left(\Omega^{a}\right)\right)^{3 \times 3}, \\
& \chi^{\varepsilon} u^{a \varepsilon} \rightarrow u^{a} \text { strongly in }\left(L^{2}\left(\Omega^{a}\right)\right)^{3}, \\
& \chi^{\varepsilon} u_{\mid \Sigma^{a}}^{a \varepsilon} \rightarrow u_{\mid \Sigma^{a}}^{a} \text { strongly in }\left(L^{2}\left(\Sigma^{a}\right)\right)^{3},
\end{aligned}
$$

so that, by virtue of (2.7), (2.9), (2.11), (4.5):

$$
\begin{aligned}
& \int_{\Omega^{a} \cap\left\{x_{3}>r^{\varepsilon}\right\}}\left[A^{a} \bar{e}^{a \varepsilon}-g^{a \varepsilon}, e^{a \varepsilon}\left(u^{a \varepsilon}\right)\right] \mathrm{d} x-\int_{\Omega^{a} \cap\left\{x_{3}>r^{\varepsilon}\right\}} f^{a \varepsilon} \cdot u^{a \varepsilon} \mathrm{d} x-\int_{\Sigma^{a} \cap\left\{x_{3}>r^{\varepsilon}\right\}} h^{a \varepsilon} \cdot u^{a \varepsilon} \mathrm{d} \sigma \\
& \rightarrow \int_{\Omega^{a}}\left[A^{a} \bar{e}^{a}-g^{a}, e^{a}\left(z^{a}\right)\right] \mathrm{d} x-\int_{\Omega^{a}} f^{a} \cdot u^{a} \mathrm{~d} x-\int_{\Sigma^{a}} h^{a} \cdot u^{a} \mathrm{~d} \sigma .
\end{aligned}
$$

For the terms in $\Omega^{a} \cap\left\{0<x_{3}<r^{\varepsilon}\right\}$ and $\Sigma^{a} \cap\left\{0<x_{3}<r^{\varepsilon}\right\}$, it is clear, from (2.7), (2.11) and from the uniform boundedness of $u^{a \varepsilon}$, that:

$$
\int_{\Omega^{a} \cap\left\{0<x_{3}<r^{\varepsilon}\right\}} f^{a \varepsilon} \cdot u^{a \varepsilon} \mathrm{d} x-\int_{\Sigma^{a} \cap\left\{0<x_{3}<r^{\varepsilon}\right\}} h^{a \varepsilon} \cdot u^{a \varepsilon} \mathrm{d} \sigma \rightarrow 0 .
$$


Hence it remains to show that:

$$
\int_{\Omega^{a} \cap\left\{0<x_{3}<r^{\varepsilon}\right\}}\left[A^{a} \bar{e}^{a \varepsilon}-g^{a \varepsilon}, e^{a \varepsilon}\left(u^{a \varepsilon}\right)\right] \mathrm{d} x \rightarrow 0 .
$$

But we have, from Cauchy-Schwarz inequality, (2.9) and (4.5):

$$
\int_{\Omega^{a} \cap\left\{0<x_{3}<r^{\varepsilon}\right\}}\left[A^{a} \bar{e}^{a \varepsilon}-g^{a \varepsilon}, e^{a \varepsilon}\left(u^{a \varepsilon}\right)\right] \mathrm{d} x \leq C\left\|e^{a \varepsilon}\left(u^{a \varepsilon}\right)\right\|_{\left(L^{2}\left(\Omega^{a} \cap\left\{0<x_{3}<r^{\varepsilon}\right\}\right)\right)^{3 \times 3}},
$$

and it is enough to prove that:

$$
\left\|e^{a \varepsilon}\left(u^{a \varepsilon}\right)\right\|_{\left(L^{2}\left(\Omega^{a} \cap\left\{0<x_{3}<r^{\varepsilon}\right\}\right)\right)^{3 \times 3}} \rightarrow 0 .
$$

Then, by passing to the limit in (6.13), we get:

$$
\left\{\begin{array}{l}
\int_{\Omega^{a}}\left[A^{a} e^{a}\left(\bar{z}^{a}\right), e^{a}\left(z^{a}\right)\right] \mathrm{d} x+q \int_{\Omega^{b}}\left[A^{b} e^{b}\left(\bar{z}^{b}\right), e^{b}\left(z^{b}\right)\right] \mathrm{d} x=\int_{\Omega^{a}} f^{a} \cdot u^{a} \mathrm{~d} x+\int_{\Omega^{b}} f^{b} \cdot u^{b} \mathrm{~d} x \\
+\int_{\Omega^{a}}\left[g^{a}, e^{a}\left(z^{a}\right)\right] \mathrm{d} x+\int_{\Omega^{b}}\left[g^{b}, e^{b}\left(z^{b}\right)\right] \mathrm{d} x+\int_{\Sigma^{a}} h^{a} \cdot u^{a} \mathrm{~d} \sigma+\int_{\omega^{b}}\left(h_{+}^{b} \cdot u_{\mid x_{3}=0}^{b}+h_{-}^{b} \cdot u_{\mid x_{3}=-1}^{b}\right) \mathrm{d} x^{\prime}
\end{array}\right.
$$

for any $z$ having the regularity given in (6.8), and then, by a density argument given in Lemma 8 of the Appendix (Sect. 8.2), for any $z$ satisfying all the requirements of $\mathcal{Z}$, except the integral conditions. A fortiori, the same variational equality holds true for any $z$ in $\mathcal{Z}$, that is $\left(\bar{z}^{a}, \bar{z}^{b}\right)$ solves $(2.15)$.

Proof of (6.14). We will prove that the norms in $L^{2}\left(\left\{0<x_{3}<r^{\varepsilon}\right\}\right)$ of the terms $e_{33}\left(u^{a \varepsilon}\right), \frac{1}{\left(r^{\varepsilon}\right)^{2}} e_{\alpha \beta}\left(u^{a \varepsilon}\right)$ and $\frac{1}{r^{\varepsilon}} e_{\alpha 3}\left(u^{a \varepsilon}\right)$ tend to zero.

- Term $e_{33}\left(u^{a \varepsilon}\right)$. We easily get from (6.12) that:

$$
e_{33}\left(u^{a \varepsilon}\right)=\frac{\partial u_{3}^{a \varepsilon}}{\partial x_{3}}=\frac{1}{r^{\varepsilon}}\left(u_{3}^{a}\left(x^{\prime}, r^{\varepsilon}\right)-u_{3}^{b}\left(r^{\varepsilon} x^{\prime}\right)\right)+v_{3}^{a}\left(x^{\prime}, r^{\varepsilon}\right)-\frac{\varepsilon^{2}}{r^{\varepsilon}} w_{3}^{b}\left(r^{\varepsilon} x^{\prime}, 0\right) .
$$

The norms in $L^{2}\left(\left\{0<x_{3}<r^{\varepsilon}\right\}\right)$ of the two last terms in (6.16) tend to zero, since $v_{3}^{a}\left(x^{\prime}, r^{\varepsilon}\right)$ and $w_{3}^{b}\left(r^{\varepsilon} x^{\prime}, 0\right)$ are uniformly bounded:

$$
\begin{gathered}
\int_{0<x_{3}<r^{\varepsilon}}\left|v_{3}^{a}\left(x^{\prime}, r^{\varepsilon}\right)\right|^{2} \mathrm{~d} x \leq C r^{\varepsilon} \rightarrow 0, \\
\int_{0<x_{3}<r^{\varepsilon}}\left(\frac{\varepsilon^{2}}{r^{\varepsilon}}\right)^{2}\left|w_{3}^{b}\left(r^{\varepsilon} x^{\prime}, 0\right)\right|^{2} \mathrm{~d} x \leq C \frac{\varepsilon^{4}}{r^{\varepsilon}} \ll C \frac{\varepsilon^{2}}{r^{\varepsilon}} \rightarrow 0,
\end{gathered}
$$

since, by assumption, $\varepsilon^{2} \ll r^{\varepsilon}$. As for the first term in (6.16), it is uniformly bounded, because of the junction condition:

$$
\begin{aligned}
\frac{1}{r^{\varepsilon}}\left(u_{3}^{a}\left(x^{\prime}, r^{\varepsilon}\right)-u_{3}^{b}\left(r^{\varepsilon} x^{\prime}\right)\right) & =\frac{1}{r^{\varepsilon}}\left(u_{3}^{a}\left(x^{\prime}, 0\right)+\int_{0}^{r^{\varepsilon}} \frac{\partial u_{3}^{a}}{\partial x_{3}}\left(x^{\prime}, t\right) \mathrm{d} t-u_{3}^{b}(0)-\int_{0}^{r^{\varepsilon}} \nabla u_{3}^{b}\left(t x^{\prime}\right) \cdot x^{\prime} \mathrm{d} t\right) \\
& =\frac{1}{r^{\varepsilon}} \int_{0}^{r^{\varepsilon}} \frac{\partial u_{3}^{a}}{\partial x_{3}}\left(x^{\prime}, t\right) \mathrm{d} t-\frac{1}{r^{\varepsilon}} \int_{0}^{r^{\varepsilon}} \nabla u_{3}^{b}\left(t x^{\prime}\right) \cdot x^{\prime} \mathrm{d} t \leq C,
\end{aligned}
$$

and hence, its norm in $L^{2}\left(\left\{0<x_{3}<r^{\varepsilon}\right\}\right)$ tends to zero. 
- Term $\frac{1}{\left(r^{\varepsilon}\right)^{2}} e_{\alpha \beta}\left(u^{a \varepsilon}\right)$. From (6.11) we have:

$$
\frac{\partial u_{\alpha}^{a \varepsilon}}{\partial x_{\beta}}=\frac{x_{3}}{r^{\varepsilon}}\left(r^{\varepsilon} \frac{\partial v_{\alpha}^{a}}{\partial x_{\beta}}\left(x^{\prime}, r^{\varepsilon}\right)+\left(r^{\varepsilon}\right)^{2} \frac{\partial w_{\alpha}^{a}}{\partial x_{\beta}}\left(x^{\prime}, r^{\varepsilon}\right)\right)+\left(1-\frac{x_{3}}{r^{\varepsilon}}\right) \varepsilon\left(r^{\varepsilon}\right)^{2}\left(\frac{\partial \zeta_{\alpha}^{b}}{\partial x_{\beta}}\left(r^{\varepsilon} x^{\prime}\right)+\varepsilon \frac{\partial v_{\alpha}^{b}}{\partial x_{\beta}}\left(r^{\varepsilon} x^{\prime}, 0\right)\right)
$$

and hence, since $e_{\alpha \beta}\left(v^{a}\right)=0$ :

$$
\frac{1}{\left(r^{\varepsilon}\right)^{2}} e_{\alpha \beta}\left(u^{a \varepsilon}\right)=\frac{x_{3}}{r^{\varepsilon}} e_{\alpha \beta}\left(w^{a}\right)\left(x^{\prime}, r^{\varepsilon}\right)+\left(1-\frac{x_{3}}{r^{\varepsilon}}\right) \varepsilon\left(e_{\alpha \beta}\left(\zeta^{b}\right)\left(r^{\varepsilon} x^{\prime}\right)+\varepsilon e_{\alpha \beta}\left(v^{b}\right)\left(r^{\varepsilon} x^{\prime}, 0\right)\right),
$$

which gives, from the regularity of $w^{a}, \zeta^{b}$ and $v^{b}$ (see (6.8)):

$$
\left|\frac{1}{\left(r^{\varepsilon}\right)^{2}} e_{\alpha \beta}\left(u^{a \varepsilon}\right)\right| \leq C+C \varepsilon(1+\varepsilon) \leq C,
$$

and hence, the norm in $L^{2}\left(\left\{0<x_{3}<r^{\varepsilon}\right\}\right)$ of this term tends to zero.

- $\operatorname{Term} \frac{1}{r^{\varepsilon}} e_{\alpha 3}\left(u^{a \varepsilon}\right)$. From (6.11) we have:

$$
\frac{\partial u_{\alpha}^{a \varepsilon}}{\partial x_{3}}=\frac{1}{r^{\varepsilon}}\left(u_{\alpha}^{a}\left(r^{\varepsilon}\right)+r^{\varepsilon} v_{\alpha}^{a}\left(x^{\prime}, r^{\varepsilon}\right)+\left(r^{\varepsilon}\right)^{2} w_{\alpha}^{a}\left(x^{\prime}, r^{\varepsilon}\right)\right)-\varepsilon\left(\zeta_{\alpha}^{b}\left(r^{\varepsilon} x^{\prime}\right)+\varepsilon v_{\alpha}^{b}\left(r^{\varepsilon} x^{\prime}, 0\right)\right),
$$

and, from (6.12):

$$
\frac{\partial u_{3}^{a \varepsilon}}{\partial x_{\alpha}}=\frac{x_{3}}{r^{\varepsilon}}\left(\frac{\partial u_{3}^{a}}{\partial x_{\alpha}}\left(x^{\prime}, r^{\varepsilon}\right)+r^{\varepsilon} \frac{\partial v_{3}^{a}}{\partial x_{\alpha}}\left(x^{\prime}, r^{\varepsilon}\right)\right)+\left(1-\frac{x_{3}}{r^{\varepsilon}}\right) r^{\varepsilon}\left(\frac{\partial u_{3}^{b}}{\partial x_{\alpha}}\left(r^{\varepsilon} x^{\prime}\right)+\varepsilon^{2} \frac{\partial w_{3}^{b}}{\partial x_{\alpha}}\left(r^{\varepsilon} x^{\prime}, 0\right)\right),
$$

so that:

$$
\frac{2}{r^{\varepsilon}} e_{\alpha 3}\left(u^{a \varepsilon}\right)=\frac{1}{r^{\varepsilon}}\left(\frac{\partial u_{\alpha}^{a \varepsilon}}{\partial x_{3}}+\frac{\partial u_{3}^{a \varepsilon}}{\partial x_{\alpha}}\right)=T_{1}+T_{2}+T_{3}+T_{4}
$$

with:

$$
\begin{aligned}
& T_{1}=\frac{1}{\left(r^{\varepsilon}\right)^{2}}\left(u_{\alpha}^{a}\left(r^{\varepsilon}\right)+x_{3} \frac{\partial u_{3}^{a}}{\partial x_{\alpha}}\left(x^{\prime}, r^{\varepsilon}\right)\right), \\
& T_{2}=\frac{1}{r^{\varepsilon}}\left(v_{\alpha}^{a}\left(x^{\prime}, r^{\varepsilon}\right)-\varepsilon\left(\zeta_{\alpha}^{b}\left(r^{\varepsilon} x^{\prime}\right)+\varepsilon v_{\alpha}^{b}\left(r^{\varepsilon} x^{\prime}, 0\right)\right)\right), \\
& T_{3}=w_{\alpha}^{a}\left(x^{\prime}, r^{\varepsilon}\right), \\
& T_{4}=\frac{x_{3}}{r^{\varepsilon}} \frac{\partial u_{3}^{a}}{\partial x_{\alpha}}\left(x^{\prime}, r^{\varepsilon}\right)+\left(1-\frac{x_{3}}{r^{\varepsilon}}\right)\left(\frac{\partial u_{3}^{b}}{\partial x_{\alpha}}\left(r^{\varepsilon} x^{\prime}\right)+\varepsilon^{2} \frac{\partial w_{3}^{b}}{\partial x_{\alpha}}\left(r^{\varepsilon} x^{\prime}, 0\right)\right) .
\end{aligned}
$$

We will show that the norm in $L^{2}\left(\left\{0<x_{3}<r^{\varepsilon}\right\}\right)$ of each term tends to zero.

$\circ \operatorname{Term} T_{1}$. As $u_{\alpha}^{a}(0)=0$, we have:

$$
u_{\alpha}^{a}\left(r^{\varepsilon}\right)=\int_{0}^{r^{\varepsilon}} \frac{\mathrm{d} u_{\alpha}^{a}}{\mathrm{~d} x_{3}}(t) \mathrm{d} t
$$


and, as $e_{\alpha 3}\left(u^{a}\right)=0$ :

$$
x_{3} \frac{\partial u_{3}^{a}}{\partial x_{\alpha}}\left(x^{\prime}, r^{\varepsilon}\right)=-x_{3} \frac{\mathrm{d} u_{\alpha}^{a}}{\mathrm{~d} x_{3}}\left(r^{\varepsilon}\right)=-\frac{x_{3}}{r^{\varepsilon}} \int_{0}^{r^{\varepsilon}} \frac{\mathrm{d} u_{\alpha}^{a}}{\mathrm{~d} x_{3}}(t) \mathrm{d} t+\frac{x_{3}}{r^{\varepsilon}} \int_{0}^{r^{\varepsilon}}\left(\frac{\mathrm{d} u_{\alpha}^{a}}{\mathrm{~d} x_{3}}(t)-\frac{\mathrm{d} u_{\alpha}^{a}}{\mathrm{~d} x_{3}}\left(r^{\varepsilon}\right)\right) \mathrm{d} t,
$$

so that, since $\frac{\mathrm{d} u_{\alpha}^{a}}{\mathrm{~d} x_{3}}(0)=0$ :

$u_{\alpha}^{a}\left(r^{\varepsilon}\right)+x_{3} \frac{\partial u_{3}^{a}}{\partial x_{\alpha}}\left(x^{\prime}, r^{\varepsilon}\right)=\left(1-\frac{x_{3}}{r^{\varepsilon}}\right) \int_{0}^{r^{\varepsilon}} \frac{\mathrm{d} u_{\alpha}^{a}}{\mathrm{~d} x_{3}}(t) \mathrm{d} t+\frac{x_{3}}{r^{\varepsilon}} \int_{0}^{r^{\varepsilon}}\left(\frac{\mathrm{d} u_{\alpha}^{a}}{\mathrm{~d} x_{3}}(t)-\frac{\mathrm{d} u_{\alpha}^{a}}{\mathrm{~d} x_{3}}\left(r^{\varepsilon}\right)\right) \mathrm{d} t=\int_{0}^{r^{\varepsilon}} \int_{0}^{t} \frac{\mathrm{d}^{2} u_{\alpha}^{a}}{\mathrm{~d} x_{3}^{2}}(\tau) \mathrm{d} \tau \mathrm{d} t$,

and, from the regularity of $u_{\alpha}^{a}$ :

$$
\left|T_{1}\right|=\frac{1}{\left(r^{\varepsilon}\right)^{2}}\left|u_{\alpha}^{a}\left(r^{\varepsilon}\right)+x_{3} \frac{\partial u_{3}^{a}}{\partial x_{\alpha}}\left(x^{\prime}, r^{\varepsilon}\right)\right| \leq \frac{1}{\left(r^{\varepsilon}\right)^{2}} \int_{0}^{r^{\varepsilon}} \int_{0}^{t}\left|\frac{\mathrm{d}^{2} u_{\alpha}^{a}}{\mathrm{~d} x_{3}^{2}}(\tau)\right| \mathrm{d} \tau \mathrm{d} t \leq C,
$$

so that the norm in $L^{2}\left(\left\{0<x_{3}<r^{\varepsilon}\right\}\right)$ of $T_{1}$ tends to zero.

○ Term $T_{2}$. We have:

But, as $c(0)=0$ :

$$
T_{2}=\frac{1}{r^{\varepsilon}} v_{\alpha}^{a}\left(x^{\prime}, r^{\varepsilon}\right)-\frac{\varepsilon}{r^{\varepsilon}}\left(\zeta_{\alpha}^{b}\left(r^{\varepsilon} x^{\prime}\right)+\varepsilon v_{\alpha}^{b}\left(r^{\varepsilon} x^{\prime}, 0\right)\right)
$$

$$
\left|\frac{1}{r^{\varepsilon}} v_{\alpha}^{a}\left(x^{\prime}, r^{\varepsilon}\right)\right| \leq \frac{C}{r^{\varepsilon}}\left|c\left(r^{\varepsilon}\right)\right| \leq C
$$

and the norm of this term in $L^{2}\left(\left\{0<x_{3}<r^{\varepsilon}\right\}\right)$ tends to zero. On the other hand, as $\varepsilon^{2} \ll r^{\varepsilon}$ and as $\zeta_{\alpha}^{b}$ and $v_{\alpha}^{b}$ are uniformly bounded, due to the regularity conditions (6.8), we have:

$$
\left|\frac{\varepsilon}{r^{\varepsilon}}\left(\zeta_{\alpha}^{b}\left(r^{\varepsilon} x^{\prime}\right)+\varepsilon v_{\alpha}^{b}\left(r^{\varepsilon} x^{\prime}, 0\right)\right)\right| \leq C \frac{\varepsilon}{r^{\varepsilon}}
$$

so that the norm of this term in $L^{2}\left(\left\{0<x_{3}<r^{\varepsilon}\right\}\right)$ is bounded by $C \varepsilon / \sqrt{r^{\varepsilon}}$, which tends to zero by assumption. Therefore the norm in $L^{2}\left(\left\{0<x_{3}<r^{\varepsilon}\right\}\right)$ of $T_{2}$ tends to zero.

- Terms $T_{3}$ and $T_{4}$. These terms are bounded, due to the regularity conditions (6.8), and therefore the norms in $L^{2}\left(\left\{0<x_{3}<r^{\varepsilon}\right\}\right)$ of those two terms tend to zero.

\section{Proof of Stronger convergences and proof of Corollary 1}

Actually, the stronger convergences in Theorem 1 are deduced from Corollary 1 . The proof is as follows. Taking $\bar{u}^{\varepsilon}=\left(\bar{u}^{a \varepsilon}, \bar{u}^{b \varepsilon}\right)$ as test function in the variational equation of problem (2.3), we get

$$
\left\{\begin{aligned}
\mathcal{E}^{\varepsilon} & =\int_{\Omega^{a}}\left[A^{a} \bar{e}^{a \varepsilon}, \bar{e}^{a \varepsilon}\right] \mathrm{d} x+q^{\varepsilon} \int_{\Omega^{b}}\left[A^{b} \bar{e}^{b \varepsilon}, \bar{e}^{b \varepsilon}\right] \mathrm{d} x \\
& =\int_{\Omega^{a}} f^{a \varepsilon} \cdot \bar{u}^{a \varepsilon} \mathrm{d} x+\int_{\Omega^{b}} f^{b \varepsilon} \cdot \bar{u}^{b \varepsilon} \mathrm{d} x+\int_{\Omega^{a}}\left[g^{a \varepsilon}, \bar{e}^{a \varepsilon}\right] \mathrm{d} x+\int_{\Omega^{b}}\left[g^{b \varepsilon}, \bar{e}^{b \varepsilon}\right] \mathrm{d} x \\
& +\int_{\Sigma^{a}} h^{a \varepsilon} \cdot \bar{u}^{a \varepsilon} \mathrm{d} \sigma+\int_{\omega^{b}}\left(h_{+}^{b \varepsilon} \cdot \bar{u}_{\mid x_{3}=0}^{b \varepsilon}+h_{-}^{b \varepsilon} \cdot \bar{u}_{\mid x_{3}=-1}^{b \varepsilon}\right) \mathrm{d} x^{\prime} .
\end{aligned}\right.
$$

We are going to pass to the limit in the right-hand side of the above equality. 
- If $0<q<+\infty$, we have, from the convergences already proved in Theorem 1 and from classical compactness arguments,

$$
\begin{aligned}
& \left(\bar{e}^{a \varepsilon}, \bar{e}^{b \varepsilon}\right) \rightarrow\left(\bar{e}^{a}, \bar{e}^{b}\right) \quad \text { weakly in }\left(L^{2}\left(\Omega^{a}\right)\right)^{3 \times 3} \times\left(L^{2}\left(\Omega^{b}\right)\right)^{3 \times 3}, \\
& \left(\bar{u}^{a \varepsilon}, \bar{u}^{b \varepsilon}\right) \rightarrow\left(\bar{u}^{a}, \bar{u}^{b}\right) \quad \text { strongly in }\left(L^{2}\left(\Omega^{a}\right)\right)^{3} \times\left(L^{2}\left(\Omega^{b}\right)\right)^{3}, \\
& \bar{u}_{\mid \Sigma^{a}}^{a \varepsilon} \rightarrow \bar{u}_{\mid \Sigma^{a}}^{a} \quad \text { strongly in }\left(L^{2}\left(\Sigma^{a}\right)\right)^{3}, \\
& \bar{u}_{\mid x_{3}=0}^{b \varepsilon} \rightarrow \bar{u}_{\mid x_{3}=0}^{b} \quad \text { strongly in }\left(L^{2}\left(\omega^{b}\right)\right)^{3}, \\
& \bar{u}_{\mid x_{3}=-1}^{b \varepsilon} \rightarrow \bar{u}_{\mid x_{3}=-1}^{b} \quad \text { strongly in }\left(L^{2}\left(\omega^{b}\right)\right)^{3} .
\end{aligned}
$$

If $\left(g^{a \varepsilon}, g^{b \varepsilon}\right)$ tends to $\left(g^{a}, g^{b}\right)$ strongly in $\left(L^{2}\left(\Omega^{a}\right)\right)^{3 \times 3} \times\left(L^{2}\left(\Omega^{b}\right)\right)^{3 \times 3}$, it follows that:

$$
\begin{aligned}
\mathcal{E}^{\varepsilon}= & \int_{\Omega^{a}} f^{a \varepsilon} \cdot \bar{u}^{a \varepsilon} \mathrm{d} x+\int_{\Omega^{b}} f^{b \varepsilon} \cdot \bar{u}^{b \varepsilon} \mathrm{d} x+\int_{\Omega^{a}}\left[g^{a \varepsilon}, \bar{e}^{a \varepsilon}\right] \mathrm{d} x+\int_{\Omega^{b}}\left[g^{b \varepsilon}, \bar{e}^{b \varepsilon}\right] \mathrm{d} x \\
& +\int_{\Sigma^{a}} h^{a \varepsilon} \cdot \bar{u}^{a \varepsilon} \mathrm{d} \sigma+\int_{\omega^{b}}\left(h_{+}^{b \varepsilon} \cdot \bar{u}_{\mid x_{3}=0}^{b \varepsilon}+h_{-}^{b \varepsilon} \cdot \bar{u}_{\mid x_{3}=-1}^{b \varepsilon}\right) \mathrm{d} x^{\prime} \\
& \longrightarrow \int_{\Omega^{a}} f^{a} \cdot \bar{u}^{a} \mathrm{~d} x+\int_{\Omega^{b}} f^{b} \cdot \bar{u}^{b} \mathrm{~d} x+\int_{\Omega^{a}}\left[g^{a}, \bar{e}^{a}\right] \mathrm{d} x+\int_{\Omega^{b}}\left[g^{b}, \bar{e}^{b}\right] \mathrm{d} x \\
& +\int_{\Sigma^{a}} h^{a} \cdot \bar{u}^{a} \mathrm{~d} \sigma+\int_{\omega^{b}}\left(h_{+}^{b} \cdot \bar{u}_{+\mid x_{3}=0}^{b}+h_{-}^{b} \cdot \bar{u}_{\mid x_{3}=-1}^{b}\right) \mathrm{d} x^{\prime} \\
= & \int_{\Omega^{a}}\left[A^{a} \bar{e}^{a}, \bar{e}^{a}\right] \mathrm{d} x+q \int_{\Omega^{b}}\left[A^{b} \bar{e}^{b}, \bar{e}^{b}\right] \mathrm{d} x=\mathcal{E},
\end{aligned}
$$

which proves the first part of Corollary 1. Moreover, we get, from the convergence of $\mathcal{E}^{\varepsilon}$ to $\mathcal{E}$ and from a classical lower semicontinuity argument:

$$
\begin{aligned}
0 & =\liminf \left(\int_{\Omega^{a}}\left[A^{a} \bar{e}^{a \varepsilon}, \bar{e}^{a \varepsilon}\right] \mathrm{d} x-\int_{\Omega^{a}}\left[A^{a} \bar{e}^{a}, \bar{e}^{a}\right] \mathrm{d} x+q^{\varepsilon} \int_{\Omega^{b}}\left[A^{b} \bar{e}^{b \varepsilon}, \bar{e}^{b \varepsilon}\right] \mathrm{d} x-q \int_{\Omega^{b}}\left[A^{b} \bar{e}^{b}, \bar{e}^{b}\right] \mathrm{d} x\right) \\
& \geq \liminf \left(\int_{\Omega^{a}}\left[A^{a} \bar{e}^{a \varepsilon}, \bar{e}^{a \varepsilon}\right] \mathrm{d} x-\int_{\Omega^{a}}\left[A^{a} \bar{e}^{a}, \bar{e}^{a}\right] \mathrm{d} x\right)+\liminf \left(q^{\varepsilon} \int_{\Omega^{b}}\left[A^{b} \bar{e}^{b \varepsilon}, \bar{e}^{b \varepsilon}\right] \mathrm{d} x-q \int_{\Omega^{b}}\left[A^{b} \bar{e}^{b}, \bar{e}^{b}\right] \mathrm{d} x\right) \\
& =\liminf \left(\int_{\Omega^{a}}\left[A^{a} \bar{e}^{a \varepsilon}, \bar{e}^{a \varepsilon}\right] \mathrm{d} x-\int_{\Omega^{a}}\left[A^{a} \bar{e}^{a}, \bar{e}^{a}\right] \mathrm{d} x\right)+\liminf q\left(\int_{\Omega^{b}}\left[A^{b} \bar{e}^{b \varepsilon}, \bar{e}^{b \varepsilon}\right] \mathrm{d} x-\int_{\Omega^{b}}\left[A^{b} \bar{e}^{b}, \bar{e}^{b}\right] \mathrm{d} x\right) \geq 0,
\end{aligned}
$$

which gives, up to extraction of a new subsequence,

$$
\begin{aligned}
& \int_{\Omega^{a}}\left[A^{a} \bar{e}^{a \varepsilon}, \bar{e}^{a \varepsilon}\right] \mathrm{d} x \longrightarrow \int_{\Omega^{a}}\left[A^{a} \bar{e}^{a}, \bar{e}^{a}\right] \mathrm{d} x \\
& \int_{\Omega^{b}}\left[A^{b} \bar{e}^{b \varepsilon}, \bar{e}^{b \varepsilon}\right] \mathrm{d} x \longrightarrow \int_{\Omega^{b}}\left[A^{b} \bar{e}^{b}, \bar{e}^{b}\right] \mathrm{d} x .
\end{aligned}
$$


448

A. GAUDiello ET $A L$.

It follows that:

$$
\begin{aligned}
C\left\|\bar{e}^{a \varepsilon}-\bar{e}^{a}\right\|_{\left(L^{2}\left(\Omega^{a}\right)\right)^{3 \times 3}}^{2} & \leq \int_{\Omega^{a}}\left[A^{a}\left(\bar{e}^{a \varepsilon}-\bar{e}^{a}\right),\left(\bar{e}^{a \varepsilon}-\bar{e}^{a}\right)\right] \mathrm{d} x \\
& =\int_{\Omega^{a}}\left[A^{a} \bar{e}^{a \varepsilon}, \bar{e}^{a \varepsilon}\right] \mathrm{d} x+\int_{\Omega^{a}}\left[A^{a} \bar{e}^{a}, \bar{e}^{a}\right] \mathrm{d} x-\int_{\Omega^{a}}\left[A^{a} \bar{e}^{a \varepsilon}, \bar{e}^{a}\right] \mathrm{d} x-\int_{\Omega^{a}}\left[A^{a} \bar{e}^{a}, \bar{e}^{a \varepsilon}\right] \mathrm{d} x \longrightarrow 0,
\end{aligned}
$$

and hence $\bar{e}^{a \varepsilon}$ tends to $\bar{e}^{a}$ strongly in $\left(L^{2}\left(\Omega^{a}\right)\right)^{3 \times 3}$. Therefore $e\left(\bar{u}^{a \varepsilon}\right)$ tends to $e\left(\bar{u}^{a}\right)$ strongly in $\left(L^{2}\left(\Omega^{a}\right)\right)^{3 \times 3}$ and then, from Korn's inequality, $\bar{u}^{a \varepsilon}$ tends to $\bar{u}^{a}$ strongly in $\left(H^{1}\left(\Omega^{a}\right)\right)^{3}$. By the same proof, $\bar{e}^{b \varepsilon}$ tends to $\bar{e}^{b}$ strongly in $\left(L^{2}\left(\Omega^{b}\right)\right)^{3 \times 3}$ and $\bar{u}^{b \varepsilon}$ tends to $\bar{u}^{b}$ strongly in $\left(H^{1}\left(\Omega^{b}\right)\right)^{3}$. In conclusion, we proved the stronger convergences mentioned in Theorem 1 when $0<q<+\infty$.

- If $q=+\infty$, we have seen that:

$$
\begin{gathered}
\bar{u}^{b \varepsilon} \rightarrow \bar{u}^{b}=0 \text { strongly in }\left(H^{1}\left(\Omega^{b}\right)\right)^{3}, \\
\bar{e}^{b \varepsilon} \rightarrow \bar{e}^{b}=0 \text { strongly in }\left(L^{2}\left(\Omega^{b}\right)\right)^{3 \times 3},
\end{gathered}
$$

and, with appropriate changes in the above proof, we have, if $g^{a \varepsilon}$ tend to $g^{a}$ strongly in $\left(L^{2}\left(\Omega^{a}\right)\right)^{3 \times 3}$ :

$$
\begin{gathered}
\mathcal{E}^{\varepsilon} \longrightarrow \int_{\Omega^{a}} f^{a} \cdot \bar{u}^{a} \mathrm{~d} x+\int_{\Omega^{a}}\left[g^{a}, \bar{e}^{a}\right] \mathrm{d} x+\int_{\Sigma^{a}} h^{a} \cdot \bar{u}^{a} \mathrm{~d} \sigma=\int_{\Omega^{a}}\left[A^{a} \bar{e}^{a}, \bar{e}^{a}\right] \mathrm{d} x=\mathcal{E}_{\infty}, \\
0=\liminf \left(\int_{\Omega^{a}}\left[A^{a} \bar{e}^{a \varepsilon}, \bar{e}^{a \varepsilon}\right] \mathrm{d} x-\int_{\Omega^{a}}\left[A^{a} \bar{e}^{a}, \bar{e}^{a}\right] \mathrm{d} x+q^{\varepsilon} \int_{\Omega^{b}}\left[A^{b} \bar{e}^{b \varepsilon}, \bar{e}^{b \varepsilon}\right] \mathrm{d} x\right) \\
\geq \liminf \left(\int_{\Omega^{a}}\left[A^{a} \bar{e}^{a \varepsilon}, \bar{e}^{a \varepsilon}\right] \mathrm{d} x-\int_{\Omega^{a}}\left[A^{a} \bar{e}^{a}, \bar{e}^{a}\right] \mathrm{d} x\right)+\liminf \left(q^{\varepsilon} \int_{\Omega^{b}}\left[A^{b} \bar{e}^{b \varepsilon}, \bar{e}^{b \varepsilon}\right] \mathrm{d} x\right) \geq 0, \\
\int_{\Omega^{a}}\left[A^{a} \bar{e}^{a \varepsilon}, \bar{e}^{a \varepsilon}\right] \mathrm{d} x \longrightarrow \int_{\Omega^{a}}\left[A^{a} \bar{e}^{a}, \bar{e}^{a}\right] \mathrm{d} x, \\
q^{\varepsilon} \int_{\Omega^{b}}\left[A^{b} \bar{e}^{b \varepsilon}, \bar{e}^{b \varepsilon}\right] \mathrm{d} x \longrightarrow 0, \\
\bar{e}^{a \varepsilon} \rightarrow \bar{e}^{a} \text { strongly in }\left(L^{2}\left(\Omega^{a}\right)\right)^{3 \times 3}, \\
\sqrt{q^{\varepsilon}} \bar{e}^{b \varepsilon} \rightarrow 0 \text { strongly in }\left(L^{2}\left(\Omega^{a}\right)\right)^{3 \times 3}, \\
\bar{u}^{a \varepsilon} \rightarrow \bar{u}^{a} \text { strongly in }\left(H^{1}\left(\Omega^{a}\right)\right)^{3} .
\end{gathered}
$$

- If $q=0$, we have, with $\tilde{u}^{\varepsilon}=q^{\varepsilon} \bar{u}^{\varepsilon}, \tilde{e}^{\varepsilon}=q^{\varepsilon} \bar{e}^{\varepsilon}$ :

$$
\left\{\begin{aligned}
\mathcal{E}^{\varepsilon} & =\frac{1}{q^{\varepsilon}} \int_{\Omega^{a}}\left[A^{a} \tilde{e}^{a \varepsilon}, \tilde{e}^{a \varepsilon}\right] \mathrm{d} x+\int_{\Omega^{b}}\left[A^{b} \tilde{e}^{b \varepsilon}, \tilde{e}^{b \varepsilon}\right] \mathrm{d} x \\
& =\int_{\Omega^{a}} f^{a \varepsilon} \cdot \tilde{u}^{a \varepsilon} \mathrm{d} x+\int_{\Omega^{b}} f^{b \varepsilon} \cdot \tilde{u}^{b \varepsilon} \mathrm{d} x+\int_{\Omega^{a}}\left[g^{a \varepsilon}, \tilde{e}^{a \varepsilon}\right] \mathrm{d} x+\int_{\Omega^{b}}\left[g^{b \varepsilon}, \tilde{e}^{b \varepsilon}\right] \mathrm{d} x \\
& +\int_{\Sigma^{a}} h^{a \varepsilon} \cdot \tilde{u}^{a \varepsilon} \mathrm{d} \sigma+\int_{\omega^{b}}\left(h_{+}^{b \varepsilon} \cdot \tilde{u}_{\mid x_{3}=0}^{b \varepsilon}+h_{-}^{b \varepsilon} \cdot \tilde{u}_{\mid x_{3}=-1}^{b \varepsilon}\right) \mathrm{d} x^{\prime}
\end{aligned}\right.
$$




$$
\begin{gathered}
\tilde{u}^{a \varepsilon} \rightarrow \bar{u}^{a}=0 \text { strongly in }\left(H^{1}\left(\Omega^{a}\right)\right)^{3}, \\
\tilde{e}^{a \varepsilon} \rightarrow \bar{e}^{a}=0 \text { strongly in }\left(L^{2}\left(\Omega^{a}\right)\right)^{3 \times 3},
\end{gathered}
$$

and we have, if $g^{b \varepsilon}$ tend to $g^{b}$ strongly in $\left(L^{2}\left(\Omega^{b}\right)\right)^{3 \times 3}$ :

$$
\begin{gathered}
\mathcal{E}^{\varepsilon} \longrightarrow \int_{\Omega^{b}} f^{b} \cdot \bar{u}^{b} \mathrm{~d} x+\int_{\Omega^{b}}\left[g^{b}, \bar{e}^{b}\right] \mathrm{d} x+\int_{\omega^{b}}\left(h_{+}^{b} \bar{u}_{\mid x_{3}=0}^{b}+h_{-}^{b} \cdot \bar{u}_{\mid x_{3}=-1}^{b}\right) \mathrm{d} x^{\prime}=\int_{\Omega^{b}}\left[A^{b} \bar{e}^{b}, \bar{e}^{b}\right] \mathrm{d} x=\mathcal{E}_{0}, \\
0=\liminf \left(\frac{1}{q^{\varepsilon}} \int_{\Omega^{a}}\left[A^{a} \tilde{e}^{a \varepsilon}, \tilde{e}^{a \varepsilon}\right] \mathrm{d} x+\int_{\Omega^{b}}\left[A^{b} \tilde{e}^{b \varepsilon}, \tilde{e}^{b \varepsilon}\right] \mathrm{d} x-\int_{\Omega^{b}}\left[A^{b} \bar{e}^{b}, \bar{e}^{b}\right] \mathrm{d} x\right) \\
\geq \liminf \left(\frac{1}{q^{\varepsilon}} \int_{\Omega^{a}}\left[A^{a} \tilde{e}^{a \varepsilon}, \tilde{e}^{a \varepsilon}\right] \mathrm{d} x\right)+\liminf \left(\int_{\Omega^{b}}\left[A^{b} \tilde{e}^{b \varepsilon}, \tilde{e}^{b \varepsilon}\right] \mathrm{d} x-\int_{\Omega^{b}}\left[A^{b} \bar{e}^{b}, \bar{e}^{b}\right] \mathrm{d} x\right) \geq 0, \\
\int_{\Omega^{b}}\left[A^{b} \tilde{e}^{b \varepsilon}, \tilde{e}^{b \varepsilon}\right] \mathrm{d} x \longrightarrow \int_{\Omega^{b}}\left[A^{a} \bar{e}^{b}, \bar{e}^{b}\right] \mathrm{d} x \\
\frac{1}{q^{\varepsilon}} \int_{\Omega^{a}}\left[A^{a} \tilde{e}^{a \varepsilon}, \tilde{e}^{a \varepsilon}\right] \mathrm{d} x \longrightarrow 0, \\
q^{\varepsilon} \bar{e}^{b \varepsilon}=\tilde{e}^{b \varepsilon} \rightarrow \bar{e}^{b} \text { strongly in }\left(L^{2}\left(\Omega^{b}\right)\right)^{3 \times 3}, \\
\sqrt{q^{\varepsilon}} \bar{e}^{a \varepsilon}=\frac{1}{\sqrt{q^{\varepsilon}}} \tilde{e}^{a \varepsilon} \rightarrow 0 \text { strongly in }\left(L^{2}\left(\Omega^{b}\right)\right)^{3 \times 3}, \\
q^{\varepsilon} \bar{u}^{b \varepsilon} \rightarrow \bar{u}^{b} \text { strongly in }\left(H^{1}\left(\Omega^{b}\right)\right)^{3} .
\end{gathered}
$$

\section{ApPENDix}

\subsection{The definitions of $\left(v^{a}, w^{a}\right)$ and $\left(v^{b}, w^{b}\right)$ as suitable limits}

For the convenience of the reader, we give in this appendix a sketch of the proof of the following result, mentionned in Section 4.2 (for thin cylinders, a complete proof is given in [28]). The case of plates is analogous and simpler.

Lemma 4. (i) Let $\left\{u^{\varepsilon}\right\}_{\varepsilon}$ be a sequence in $\left(H^{1}\left(\Omega^{a}\right)\right)^{3}$ such that $u^{\varepsilon}=0$ on $T^{a}=\omega^{a} \times\{1\}$ and:

$$
\left\{e^{a \varepsilon}\left(u^{\varepsilon}\right)\right\}_{\varepsilon} \text { is bounded in }\left(L^{2}\left(\Omega^{a}\right)\right)^{3 \times 3} .
$$

Let $\mathcal{W}^{a}$ be the space defined in Section 2.2 and let:

$$
\begin{array}{r}
\mathcal{V}_{-}^{a}=\left\{v^{a} \in\left(H^{1}\left(\Omega^{a}\right)\right)^{2} \times L^{2}\left(0,1 ; H^{1}\left(\omega^{a}\right)\right), \quad \exists c \in H^{1}(0,1), \quad c(1)=0, \quad v_{1}^{a}=-c x_{2}, \quad v_{2}^{a}=c x_{1},\right. \\
\left.\int_{\omega^{a}} v_{3}^{a}\left(x^{\prime}, x_{3}\right) \mathrm{d} x^{\prime}=0, \quad \text { for } \text { a.e. } \quad x_{3} \in(0,1)\right\},
\end{array}
$$

(note that $\mathcal{V}_{-}^{a}$ satisfies the same requirements as $\mathcal{V}^{a}$, in Section 2.2 , except $\left.c(0)=0\right)$. Then there exists a pair 
$\left(v^{a}, w^{a}\right) \in \mathcal{V}_{-}^{a} \times \mathcal{W}^{a}$ such that for all $\alpha, \beta=1,2$ :

$$
\begin{gathered}
\frac{1}{r^{\varepsilon}} e_{\alpha 3}\left(u^{\varepsilon}\right) \rightarrow e_{\alpha 3}\left(v^{a}\right) \text { weakly in } L^{2}\left(\Omega^{a}\right), \\
\frac{1}{\left(r^{\varepsilon}\right)^{2}} e_{\alpha \beta}\left(u^{\varepsilon}\right) \rightarrow e_{\alpha \beta}\left(w^{a}\right) \text { weakly in } L^{2}\left(\Omega^{a}\right) .
\end{gathered}
$$

Morever, denoting by $\left(c, v_{3}^{a}\right)$ the couple defining $v^{a}$ and setting:

$$
\begin{gathered}
c^{\varepsilon}\left(x_{3}\right)=\frac{\int_{\omega^{a}}\left(x_{1} u_{2}^{\varepsilon}\left(x^{\prime}, x_{3}\right)-x_{2} u_{1}^{\varepsilon}\left(x^{\prime}, x_{3}\right)\right) \mathrm{d} x^{\prime}}{r^{\varepsilon} \int_{\omega^{a}}\left(x_{1}^{2}+x_{2}^{2}\right) \mathrm{d} x^{\prime}}, \\
v_{3}^{\varepsilon}=\frac{u_{3}^{\varepsilon}}{r^{\varepsilon}}-\frac{1}{\left|\omega^{a}\right|} \int_{\omega^{a}} \frac{u_{3}^{\varepsilon}}{r^{\varepsilon}} \mathrm{d} x^{\prime}+\frac{1}{\left|\omega^{a}\right|} \sum_{\alpha} x_{\alpha} \frac{\mathrm{d}}{\mathrm{d} x_{3}} \int_{\omega^{a}} u_{\alpha}^{\varepsilon} \mathrm{d} x^{\prime},
\end{gathered}
$$

we have:

$$
c^{\varepsilon} \rightarrow c \text { strongly in } L^{2}(0,1)
$$

$$
v_{3}^{\varepsilon} \rightarrow v_{3}^{a} \text { weakly in } H^{-1}\left(0,1 ; H^{1}\left(\omega^{a}\right)\right)
$$

Finally, setting:

$$
d_{\alpha}^{\varepsilon}\left(x_{3}\right)=\frac{1}{\left|\omega^{a}\right|} \int_{\omega^{a}} \frac{u_{\alpha}^{\varepsilon}\left(x^{\prime}, x_{3}\right)}{r^{\varepsilon}} \mathrm{d} x^{\prime}
$$

and $x_{1}^{R}=-x_{2}, x_{2}^{R}=x_{1}$, we have:

$$
\frac{u_{\alpha}^{\varepsilon}}{\left(r^{\varepsilon}\right)^{2}}-\frac{1}{r^{\varepsilon}}\left(c^{\varepsilon} x_{\alpha}^{R}+d_{\alpha}^{\varepsilon}\right) \rightarrow w_{\alpha}^{a} \text { weakly in } L^{2}\left(0,1 ; H^{1}\left(\omega^{a}\right)\right) .
$$

(ii) If $\left\{u^{\varepsilon}\right\}_{\varepsilon}$ is a sequence in $\left(H^{1}\left(\Omega^{b}\right)\right)^{3}$ such that $u^{\varepsilon}=0$ on $\Sigma^{b}=\partial \omega^{b} \times(-1,0)$ and:

$$
\left\{e^{b \varepsilon}\left(u^{\varepsilon}\right)\right\}_{\varepsilon} \text { is bounded in }\left(L^{2}\left(\Omega^{b}\right)\right)^{3 \times 3},
$$

then there exists a pair $\left(v^{b}, w^{b}\right) \in \mathcal{V}^{b} \times \mathcal{W}^{b}$ such that for all $\alpha=1,2$ :

$$
\begin{aligned}
& \frac{1}{\varepsilon} e_{\alpha 3}\left(u^{\varepsilon}\right) \rightarrow e_{\alpha 3}\left(v^{b}\right) \text { weakly in } L^{2}\left(\Omega^{b}\right), \\
& \frac{1}{\varepsilon^{2}} e_{33}\left(u^{\varepsilon}\right) \rightarrow e_{33}\left(w^{b}\right) \text { weakly in } L^{2}\left(\Omega^{b}\right) .
\end{aligned}
$$

In addition, we have:

$$
\frac{u_{\alpha}^{\varepsilon}}{\varepsilon}-\tilde{u}_{\alpha}^{\varepsilon}-\int_{-1}^{0}\left(\frac{u_{\alpha}^{\varepsilon}}{\varepsilon}-\tilde{u}_{\alpha}^{\varepsilon}\right) \mathrm{d} x_{3} \rightarrow v_{\alpha}^{b} \text { weakly in } L^{2}\left(\omega^{b} ; H^{1}(-1,0)\right), \text { for } \alpha=1,2,
$$

with $\tilde{u}^{\varepsilon}$ defined by:

Moreover:

$$
\tilde{u}_{\alpha}^{\varepsilon}=-\int_{0}^{x_{3}} \frac{1}{\varepsilon} \frac{\partial u_{3}^{\varepsilon}}{\partial x_{\alpha}}\left(x^{\prime}, s\right) \mathrm{d} s .
$$

$$
\frac{u_{3}^{\varepsilon}}{\varepsilon^{2}}-\int_{-1}^{0} \frac{u_{3}^{\varepsilon}}{\varepsilon^{2}} \mathrm{~d} x_{3} \rightarrow w_{3}^{b} \text { weakly in } L^{2}\left(\omega^{b} ; H^{1}(-1,0)\right) .
$$


Proof of (i). We use the following decomposition and estimate, whose proof may be found for instance in [20,21]: there exists a positive constant $C$ such that, for every $u$ in $L^{2}\left(0,1 ; H^{1}\left(\omega^{a}\right)\right)^{2}$, there exist $\bar{u}$ and $\hat{u}$ satisfying:

$$
\left\{\begin{array}{l}
u=\bar{u}+\hat{u}, \\
\int_{\omega^{a}} \bar{u}_{\alpha}\left(x^{\prime}, x_{3}\right) \mathrm{d} x^{\prime}=0, \quad \int_{\omega^{a}}\left(x_{1} \bar{u}_{2}\left(x^{\prime}, x_{3}\right)-x_{2} \bar{u}_{1}\left(x^{\prime}, x_{3}\right)\right) \mathrm{d} x^{\prime}=0 \\
e_{\alpha \beta}(\hat{u})=0, \quad \forall \alpha, \beta=1,2, \\
\|\bar{u}\|_{\left(L^{2}\left(0,1 ; H^{1}\left(\omega^{a}\right)\right)\right)^{2}} \leq C \sum_{\alpha, \beta}\left\|e_{\alpha \beta}(u)\right\|_{L^{2}\left(\Omega^{a}\right)} .
\end{array}\right.
$$

The function $\hat{u}$ is a rigid displacement:

$$
\hat{u}_{\alpha}\left(x^{\prime}, x_{3}\right)=c\left(x_{3}\right) x_{\alpha}^{R}+d_{\alpha}\left(x_{3}\right),
$$

with $x_{1}^{R}=-x_{2}, x_{2}^{R}=x_{1}$ ( $R$ for "rotation" $)$. Applying (8.15) and (8.17) to $u=\frac{1}{r^{\varepsilon}}\left(u_{1}^{\varepsilon}, u_{2}^{\varepsilon}\right)$, we get:

$$
\frac{1}{r^{\varepsilon}} u_{\alpha}^{\varepsilon}=\bar{u}_{\alpha}^{\varepsilon}+\hat{u}_{\alpha}^{\varepsilon}, \text { with } \hat{u}_{\alpha}^{\varepsilon}=c^{\varepsilon}\left(x_{3}\right) x_{\alpha}^{R}+d_{\alpha}^{\varepsilon}\left(x_{3}\right) .
$$

One can check easily that the functions $c^{\varepsilon}$ and $d_{\alpha}^{\varepsilon}$ are given in terms of $u^{\varepsilon}$ by the formulae (8.4) and (8.8). From (8.16), we obtain:

Setting $w_{\alpha}^{\varepsilon}=\bar{u}_{\alpha}^{\varepsilon} / r^{\varepsilon}$ and using (8.1), it follows that:

$$
\left\|\bar{u}_{\alpha}^{\varepsilon}\right\|_{L^{2}\left(0,1 ; H^{1}\left(\omega^{a}\right)\right)} \leq C \sum_{\alpha, \beta}\left\|e_{\alpha \beta}\left(\frac{1}{r^{\varepsilon}} u^{\varepsilon}\right)\right\|_{L^{2}\left(\Omega^{a}\right)} .
$$

$$
\left\|w_{\alpha}^{\varepsilon}\right\|_{L^{2}\left(0,1 ; H^{1}\left(\omega^{a}\right)\right)} \leq C .
$$

So, taking a subsequence of $\varepsilon$, still denoted by the same letter, we may assume the existence of $w_{\alpha}^{a}$ such that:

$$
w_{\alpha}^{\varepsilon} \rightarrow w_{\alpha}^{a} \text { weakly in } L^{2}\left(0,1 ; H^{1}\left(\omega^{a}\right)\right), \forall \alpha=1,2,
$$

that is (8.9). Moreover it is clear that $\left(w_{1}^{\varepsilon}, w_{2}^{\varepsilon}, 0\right)$ and $w^{a}=\left(w_{1}^{a}, w_{2}^{a}, 0\right)$ belong to $\mathcal{W}^{a}$. Since (8.18) implies that:

$$
\frac{1}{\left(r^{\varepsilon}\right)^{2}} e_{\alpha \beta}\left(u^{\varepsilon}\right)=e_{\alpha \beta}\left(w^{\varepsilon}\right)
$$

we see that (8.3) is proved.

It remains to prove the convergences involving $v^{a}$. In Section 5.3, it is proved that there exists $c$ in $H^{1}(0,1)$, $c(1)=0$ such that, for a subsequence of $\varepsilon,(8.6)$ holds true. As for the other convergences involving $v^{a}$, we use again the decomposition (8.18), from which we deduce the following equality:

$$
\frac{2}{r^{\varepsilon}} e_{\alpha 3}\left(u^{\varepsilon}\right)=\frac{\partial \bar{u}_{\alpha}^{\varepsilon}}{\partial x_{3}}+\frac{\mathrm{d} c^{\varepsilon}}{\mathrm{d} x_{3}} x_{\alpha}^{R}+\frac{\mathrm{d} d_{\alpha}^{\varepsilon}}{\mathrm{d} x_{3}}+\frac{1}{r^{\varepsilon}} \frac{\partial u_{3}^{\varepsilon}}{\partial x_{\alpha}}, \quad \forall \alpha=1,2 .
$$

Now, setting

$$
v_{3}^{\varepsilon}=\frac{u_{3}^{\varepsilon}}{r^{\varepsilon}}-\frac{1}{\left|\omega^{a}\right|} \int_{\omega^{a}} \frac{u_{3}^{\varepsilon}}{r^{\varepsilon}} \mathrm{d} x^{\prime}+\sum_{\beta} x_{\beta} \frac{\mathrm{d}}{\mathrm{d} x_{3}} d_{\beta}^{\varepsilon}\left(x_{3}\right)
$$


equality (8.19) can be written as:

$$
\frac{2}{r^{\varepsilon}} e_{\alpha 3}\left(u^{\varepsilon}\right)=\frac{\mathrm{d} c^{\varepsilon}}{\mathrm{d} x_{3}} x_{\alpha}^{R}+\frac{\partial v_{3}^{\varepsilon}}{\partial x_{\alpha}}+\frac{\partial \bar{u}_{\alpha}^{\varepsilon}}{\partial x_{3}}
$$

The following estimate is proved in [24]:

$$
\left\|v_{3}^{\varepsilon}\right\|_{H^{-1}\left(0,1 ; H^{1}\left(\omega^{a}\right)\right)} \leq C\left(\sum_{\alpha \beta}\left\|e_{\alpha \beta}\left(\frac{u^{\varepsilon}}{r^{\varepsilon}}\right)\right\|_{L^{2}\left(\Omega^{a}\right)}+\sum_{\alpha}\left\|e_{\alpha 3}\left(\frac{u^{\varepsilon}}{r^{\varepsilon}}\right)\right\|_{L^{2}\left(\Omega^{a}\right)}\right),
$$

so that, from (8.1), the sequence $\left\{v_{3}^{\varepsilon}\right\}_{\varepsilon}$ is bounded in $H^{-1}\left(0,1 ; H^{1}\left(\omega^{a}\right)\right)$. Therefore there exists some $v_{3}^{a}$ in $H^{-1}\left(0,1 ; H^{1}\left(\omega^{a}\right)\right)$, with zero mean-value on $\omega^{a}$, such that (8.7) holds true (for a subsequence). It follows also from (8.1) that:

$$
\frac{1}{r^{\varepsilon}} e_{\alpha 3}\left(u^{\varepsilon}\right) \rightarrow \tau_{\alpha 3} \text { weakly in } L^{2}\left(\Omega^{a}\right),
$$

(again for some subsequence and some $\tau_{\alpha 3}$ in $L^{2}\left(\Omega^{a}\right)$ ). Moreover, since $w_{\alpha}^{\varepsilon}$ is bounded in $L^{2}\left(0,1 ; H^{1}\left(\omega^{a}\right)\right.$ ):

$$
\frac{\partial \bar{u}_{\alpha}^{\varepsilon}}{\partial x_{3}}=r^{\varepsilon} \frac{\partial w_{\alpha}^{\varepsilon}}{\partial x_{3}} \longrightarrow 0 \text { in the sense of distributions. }
$$

By passing to the limit in (8.20), using (8.6), (8.7), (8.21) and (8.22), we get:

$$
2 \tau_{\alpha 3}=\frac{\mathrm{d} c}{\mathrm{~d} x_{3}} x_{\alpha}^{R}+\frac{\partial}{\partial x_{\alpha}} v_{3}^{a}
$$

which, using $c \in H^{1}(0,1)$, implies that:

$$
\frac{\partial}{\partial x_{\alpha}} v_{3}^{a} \in L^{2}\left(\Omega^{a}\right)
$$

From (8.24) and from the fact that $v_{3}^{a}$ belongs to $H^{-1}\left(0,1 ; H^{1}\left(\omega^{a}\right)\right)$ and has zero mean-value on $\omega^{a}$, one deduces that $v_{3}^{a}$ belongs to $L^{2}\left(0,1 ; H^{1}\left(\omega^{a}\right)\right)$, so that $v^{a}=\left(c\left(x_{3}\right) x_{\alpha}^{R}, v_{3}^{a}\right) \in \mathcal{V}_{-}^{a}$ and satisfies (8.2).

Proof of (ii). Now we prove the analogous of the previous properties in the framework of $3 \mathrm{~d}-2 \mathrm{~d}$ reduction of dimension. This is much easier. Indeed, in order to prove (8.11) and (8.13), we consider the sequence $\left\{v_{\alpha}^{\varepsilon}\right\}_{\varepsilon}$ defined by:

$$
v_{\alpha}^{\varepsilon}=\frac{u_{\alpha}^{\varepsilon}}{\varepsilon}-\tilde{u}_{\alpha}^{\varepsilon}-\int_{-1}^{0}\left(\frac{u_{\alpha}^{\varepsilon}}{\varepsilon}-\tilde{u}_{\alpha}^{\varepsilon}\right) \mathrm{d} x_{3}
$$

and

Then we have as above:

$$
\tilde{u}^{\varepsilon}=\left(-\int_{0}^{x_{3}} \frac{1}{\varepsilon} \frac{\partial u_{3}^{\varepsilon}}{\partial x_{1}}\left(x^{\prime}, s\right) \mathrm{d} s,-\int_{0}^{x_{3}} \frac{1}{\varepsilon} \frac{\partial u_{3}^{\varepsilon}}{\partial x_{2}}\left(x^{\prime}, s\right) \mathrm{d} s, \frac{u_{3}^{\varepsilon}}{\varepsilon}\right) .
$$

$$
\frac{\partial v_{\alpha}^{\varepsilon}}{\partial x_{3}}=\frac{2}{\varepsilon} e_{\alpha 3}\left(u^{\varepsilon}\right)
$$

which is bounded in $L^{2}\left(\Omega^{b}\right)$, as a consequence of (8.10). As $v_{\alpha}^{\varepsilon}$ has mean-value zero with respect to $x_{3}$, it is bounded in $L^{2}\left(\omega^{b} ; H^{1}(-1,0)\right)$, so that $(8.13)$ holds true, i.e.:

$$
v_{\alpha}^{\varepsilon} \rightarrow v_{\alpha}^{b} \text { weakly in } L^{2}\left(\omega^{b} ; H^{1}(-1,0)\right),
$$

for some subsequence of $\varepsilon$ and for some $v_{\alpha}^{b}$ in $L^{2}\left(\omega^{b} ; H^{1}(-1,0)\right)$. 
Setting $v^{b}=\left(v_{1}^{b}, v_{2}^{b}, 0\right)$, we get:

$$
e_{\alpha 3}\left(v^{b}\right)=\frac{1}{2} \frac{\partial v_{\alpha}^{b}}{\partial x_{3}}
$$

so that we derive (8.11) from (8.25) and (8.26).

Finally we prove (8.12) and (8.14), by introducing the sequence of functions $w^{\varepsilon}$ defined by:

$$
w^{\varepsilon}=\frac{1}{\varepsilon^{2}} u_{3}^{\varepsilon}-\int_{-1}^{0} \frac{1}{\varepsilon^{2}} u_{3}^{\varepsilon} \mathrm{d} x_{3},
$$

which is bounded in $L^{2}\left(\omega^{b} ; H^{1}(-1,0)\right)$, since $\frac{\partial w^{\varepsilon}}{\partial x_{3}}=\frac{1}{\varepsilon^{2}} e_{33}\left(u^{\varepsilon}\right)$ is bounded in $L^{2}\left(\Omega^{b}\right)$, due to $(8.10)$. So, extracting a subsequence, we can find $w_{3}^{b}$ in $L^{2}\left(\omega^{b} ; H^{1}(-1,0)\right)$, with mean-value zero in $x_{3}$, such that $(8.12)$ and $(8.14)$ hold true, which completes the proof of Lemma 4.

\subsection{The density arguments}

In Section 6, we have mentionned four density arguments. These are stated in the following lemmata and proved below. This is done for the sake of completeness, since Lemmata 7 and 8 are very classical, Lemma 5 is classical and very similar to the density result proved in [13], while Lemma 6, though less classical, results from Theorem 9.1.3 of [2].

Lemma 5. Let $v \in H_{0}^{1}\left(\omega^{b}\right), 0 \in \omega^{b} \subset \mathbf{R}^{2}$. There exist a sequence of positive numbers $r^{n}$, tending to zero, and a sequence of functions $v^{n} \in H_{0}^{1}\left(\omega^{b}\right)$ such that:

$$
\begin{gathered}
v^{n}=0 \text { in the ball } B^{n} \text { of center } 0 \text { and radius } r^{n}, \\
\qquad v^{n} \rightarrow v \text { in } H_{0}^{1}\left(\omega^{b}\right) .
\end{gathered}
$$

Proof. Let $\tilde{V}=\left\{v \in \mathcal{C}^{1}\left(\overline{\omega^{b}}\right), v=0\right.$ on $\left.\partial \omega^{b}\right\}$. As $\tilde{V}$ is dense in $H_{0}^{1}\left(\omega^{b}\right)$, we may restrict to $v$ in $\tilde{V}$. Then the proof goes as follows. For any integer $n$, we consider two balls $B^{n}$ and $B^{\prime n}$ in $\omega^{b} \subset \mathbf{R}^{2}$, with center 0 and respective radii $r^{n}$ and $R^{n}$, to be determined later on, and such that $0<r^{n}<R^{n}, R^{n}$ tends to zero as $n$ tends to infinity. We define $v^{n} \in H_{0}^{1}\left(\omega^{b}\right)$ by:

$$
v^{n}=0 \text { in } B^{n}, v^{n}=v \text { in } \omega^{b} \backslash B^{\prime n}, v^{n}=\left(1-\phi^{n}\right) v \text { in } B^{\prime n} \backslash B^{n},
$$

where $\phi^{n}$ is the solution of the capacity problem in $B^{\prime n} \backslash B^{n}$ :

$$
\Delta \phi^{n}=0 \text { in } B^{\prime n} \backslash B^{n}, \quad \phi^{n}=1 \text { on } \partial B^{n}, \quad \phi^{n}=0 \text { on } \partial B^{\prime n} .
$$

It is clear that $v^{n} \in W^{1, \infty}\left(\omega^{b}\right) \cap H_{0}^{1}\left(\omega^{b}\right)$ and $v^{n}=0$ in $B^{n}$. We are going to prove that, for convenient $r^{n}$ and $R^{n}, v^{n} \rightarrow v$ in $H_{0}^{1}\left(\omega^{b}\right)$. Actually, as $0 \leq \phi^{n} \leq 1$, we have: 


$$
\begin{aligned}
\left\|v^{n}-v\right\|_{H_{0}^{1}\left(\omega^{b}\right)}^{2} & =\int_{B^{\prime n}}\left|\nabla\left(v^{n}-v\right)\right|^{2} \mathrm{~d} x^{\prime} \\
& =\int_{B^{n}}|\nabla v|^{2} \mathrm{~d} x^{\prime}+\int_{B^{\prime n} \backslash B^{n}}\left|\phi^{n} \nabla v+v \nabla \phi^{n}\right|^{2} \mathrm{~d} x^{\prime} \\
& \leq \int_{B^{n}}|\nabla v|^{2} \mathrm{~d} x^{\prime}+2 \int_{B^{\prime n} \backslash B^{n}}\left|\phi^{n} \nabla v\right|^{2} \mathrm{~d} x^{\prime}+2 \int_{B^{\prime n} \backslash B^{n}}\left|v \nabla \phi^{n}\right|^{2} \mathrm{~d} x^{\prime} \\
& \leq 3 \int_{B^{\prime n}}|\nabla v|^{2} \mathrm{~d} x^{\prime}+2 \int_{B^{\prime n} \backslash B^{n}}\left|v \nabla \phi^{n}\right|^{2} \mathrm{~d} x^{\prime} \\
& \leq 3 \pi\left(R^{n}\right)^{2}\|\nabla v\|_{L^{\infty}\left(\omega^{b}\right)}^{2}+2\|v\|_{L^{\infty}\left(\omega^{b}\right)}^{2} \int_{B^{\prime n} \backslash B^{n}}\left|\nabla \phi^{n}\right|^{2} \mathrm{~d} x^{\prime} \\
& =3 \pi\left(R^{n}\right)^{2}\|\nabla v\|_{L^{\infty}\left(\omega^{b}\right)}^{2}+4 \pi\|v\|_{L^{\infty}\left(\omega^{b}\right)}^{2}\left(\log \frac{R^{n}}{r^{n}}\right)^{-1} .
\end{aligned}
$$

It is enough to take e.g. $r^{n}=1 / n^{2}$ and $R^{n}=1 / n$, in order to get $v^{n} \rightarrow v$ in $H_{0}^{1}\left(\omega^{b}\right)$.

Lemma 6. Let $v \in H_{0}^{2}\left(\omega^{b}\right), 0 \in \omega^{b} \subset \mathbf{R}^{2}, v(0)=0$. There exist a sequence of positive numbers $r^{n}$, tending to zero, and a sequence of functions $v^{n} \in H_{0}^{2}\left(\omega^{b}\right)$ such that:

$$
\begin{gathered}
v^{n}=0 \text { in the ball } B^{n} \text { of center } 0 \text { and radius } r^{n}, \\
v^{n}-v \text { weakly in } H_{0}^{2}\left(\omega^{b}\right) .
\end{gathered}
$$

Proof. • For any $v \in H_{0}^{2}\left(\omega^{b}\right)$, with $v(0)=0$, there exists $\bar{v}^{n} \in \mathcal{C}^{2}\left(\overline{\omega^{b}}\right) \cap H_{0}^{2}\left(\omega^{b}\right)$ such that $\bar{v}^{n}$ tends to $v$ in $H^{2}\left(\omega^{b}\right)$ and hence in $\mathcal{C}^{0}\left(\overline{\omega^{b}}\right)$. In particular, $\bar{v}^{n}(0)$ tends to $v(0)=0$. Setting $v^{n}=\bar{v}^{n}-\bar{v}^{n}(0) \phi$, with $\phi \in \mathcal{D}\left(\omega^{b}\right)$ and $\phi(0)=1$, it is clear that $v^{n} \in \mathcal{C}^{2}\left(\overline{\omega^{b}}\right) \cap H_{0}^{2}\left(\omega^{b}\right), v^{n}(0)=0$ and that $v^{n}$ tends to $v$ in $H^{2}\left(\omega^{b}\right)$.

- In view of the result of the previous step, we may restrict to $v$ in $\mathcal{C}^{2}\left(\overline{\omega^{b}}\right) \cap H_{0}^{2}\left(\omega^{b}\right), v(0)=0$. Let $v^{n}=v \phi^{n}$, with $\phi^{n}\left(x^{\prime}\right)=\phi\left(n\left|x^{\prime}\right|\right)$ and $\phi \in \mathcal{C}^{\infty}(\mathbf{R}), 0 \leq \phi \leq 1, \phi=0$ on $(-\infty, 1], \phi=1$ on $[2,+\infty)$. Clearly $v^{n} \in H_{0}^{2}\left(\omega^{b}\right)$, $v^{n}=0$ in the ball of center 0 and radius $1 / n$ and we have:

$$
\int_{\omega^{b}}\left|v^{n}-v\right|^{2} \mathrm{~d} x^{\prime} \leq \int_{\left|x^{\prime}\right|<\frac{2}{n}}|v|^{2} \mathrm{~d} x^{\prime} \rightarrow 0,
$$

that is $v^{n} \rightarrow v$ in $L^{2}\left(\omega^{b}\right)$. Hence the lemma is proved, as soon as we have proved that $v^{n}$ is bounded uniformly in $H_{0}^{2}\left(\omega^{b}\right)$, i.e.:

$$
\frac{\partial^{2} v^{n}}{\partial x_{\alpha} \partial x_{\beta}} \text { is bounded in } L^{2}\left(\omega^{b}\right)
$$

But we have:

$$
\frac{\partial^{2} v^{n}}{\partial x_{\alpha} \partial x_{\beta}}=v \frac{\partial^{2} \phi^{n}}{\partial x_{\alpha} \partial x_{\beta}}+\phi^{n} \frac{\partial^{2} v}{\partial x_{\alpha} \partial x_{\beta}}+\frac{\partial v}{\partial x_{\alpha}} \frac{\partial \phi^{n}}{\partial x_{\beta}}+\frac{\partial v}{\partial x_{\beta}} \frac{\partial \phi^{n}}{\partial x_{\alpha}} .
$$

The second term is obviously bounded in $L^{\infty}\left(\omega^{b}\right)$. Moreover, since:

$$
\frac{\partial \phi^{n}}{\partial x_{\alpha}}=n \phi^{\prime}\left(n\left|x^{\prime}\right|\right) \frac{x_{\alpha}}{\left|x^{\prime}\right|} \text { and } \frac{\partial^{2} \phi^{n}}{\partial x_{\alpha} \partial x_{\beta}}=n^{2} \phi^{\prime \prime}\left(n\left|x^{\prime}\right|\right) \frac{x_{\alpha} x_{\beta}}{\left|x^{\prime}\right|^{2}}+n \phi^{\prime}\left(n\left|x^{\prime}\right|\right)\left(\frac{\delta_{\alpha \beta}}{\left|x^{\prime}\right|}-\frac{x_{\alpha} x_{\beta}}{\left|x^{\prime}\right|^{3}}\right),
$$


it follows that:

$$
\begin{aligned}
& \left|\frac{\partial \phi^{n}}{\partial x_{\alpha}}\right| \leq C n, \quad\left|\frac{\partial^{2} \phi^{n}}{\partial x_{\alpha} \partial x_{\beta}}\right| \leq C n^{2}, \\
& \int_{\omega^{b}}\left|\frac{\partial v}{\partial x_{\beta}} \frac{\partial \phi^{n}}{\partial x_{\alpha}}\right|^{2} \mathrm{~d} x^{\prime} \leq C\left\|\frac{\partial v}{\partial x_{\beta}}\right\|_{\infty}^{2} \int_{\frac{1}{n}<\left|x^{\prime}\right|<\frac{2}{n}} n^{2} \mathrm{~d} x^{\prime}=C\left\|\frac{\partial v}{\partial x_{\beta}}\right\|_{\infty}^{2} \int_{1<\left|x^{\prime}\right|<2} \mathrm{~d} x^{\prime}=C, \\
& \int_{\omega^{b}}\left|v \frac{\partial^{2} \phi^{n}}{\partial x_{\alpha} \partial x_{\beta}}\right|^{2} \mathrm{~d} x^{\prime} \leq\|v\|_{L^{\infty}\left(\frac{1}{n}<\left|x^{\prime}\right|<\frac{2}{n}\right)}^{2} \int_{\frac{1}{n}<\left|x^{\prime}\right|<\frac{2}{n}} C n^{4} \mathrm{~d} x^{\prime}=C n^{2}\|v\|_{L^{\infty}\left(\frac{1}{n}<\left|x^{\prime}\right|<\frac{2}{n}\right)}^{2} .
\end{aligned}
$$

But, for $1 / n<\left|x^{\prime}\right|<2 / n,\left|v\left(x^{\prime}\right)\right| \leq C\left|x^{\prime}\right| \leq C / n$, since $v$ is regular and $v(0)=0$. It follows that:

$$
\int_{\omega^{b}}\left|v \frac{\partial^{2} \phi^{n}}{\partial x_{\alpha} \partial x_{\beta}}\right|^{2} \mathrm{~d} x^{\prime} \leq C,
$$

and finally, (8.27) holds true, which completes the proof of Lemma 6.

Lemma 7. Let $v \in L^{2}\left(\omega^{b} ; H^{1}(-1,0)\right), 0 \in \omega^{b} \subset \mathbf{R}^{2}$. There exist a sequence of positive numbers $r^{n}$, tending to zero, and a sequence of functions $v^{n}$ such that:

$$
\begin{aligned}
& v^{n} \in \mathcal{C}^{1}\left(\overline{\Omega^{b}}\right) \\
& v^{n}=0 \text { in } B^{n} \times\{0\}, B^{n} \text { denoting the ball of center } 0 \text { and radius } r^{n}, \\
& v^{n} \rightarrow v \text { in } L^{2}\left(\omega^{b} ; H^{1}(-1,0)\right) .
\end{aligned}
$$

Proof. By density of $\mathcal{C}^{1}\left(\overline{\Omega^{b}}\right)$ in $L^{2}\left(\omega^{b} ; H^{1}(-1,0)\right)$, we may restrict to $v \in \mathcal{C}^{1}\left(\overline{\Omega^{b}}\right)$. We consider a sequence $r^{n}$ of positive numbers, converging to zero, and a sequence of functions $\phi^{n}: \omega^{b} \rightarrow \mathbf{R}$, of class $\mathcal{C}^{\infty}$, with $\phi^{n}=0$ in the ball $B^{n}$ of center 0 and radius $r^{n}, \phi^{n}=1$ outside the ball $B^{\prime n}$ of center 0 and radius $2 r^{n}, 0 \leq \phi^{n} \leq 1$ in $B^{\prime n} \backslash B^{n}$. We set $v^{n}=\phi^{n} v$. Then clearly $v^{n} \in \mathcal{C}^{1}\left(\overline{\Omega^{b}}\right)$ and:

$$
\begin{aligned}
\left\|v^{n}-v\right\|_{L^{2}\left(\omega^{b} ; H^{1}(-1,0)\right)}^{2} & =\int_{\Omega^{b}}\left|v^{n}-v\right|^{2} \mathrm{~d} x+\int_{\Omega^{b}}\left|\frac{\partial}{\partial x_{3}}\left(v^{n}-v\right)\right|^{2} \mathrm{~d} x \\
& =\int_{B^{n} \times(-1,0)}|v|^{2} \mathrm{~d} x+\int_{\left(B^{\prime n} \backslash B^{n}\right) \times(-1,0)}\left|\left(1-\phi^{n}\right) v\right|^{2} \mathrm{~d} x \\
& +\int_{B^{n} \times(-1,0)}\left|\frac{\partial v}{\partial x_{3}}\right|^{2} \mathrm{~d} x+\int_{\left(B^{\prime n} \backslash B^{n}\right) \times(-1,0)}\left|\left(1-\phi^{n}\right) \frac{\partial v}{\partial x_{3}}\right|^{2} \mathrm{~d} x \\
& \leq \int_{B^{\prime n} \times(-1,0)}\left(|v|^{2}+\left|\frac{\partial v}{\partial x_{3}}\right|^{2}\right) \mathrm{d} x,
\end{aligned}
$$

which tends to zero, as soon as $r^{n}$ tends to zero.

Lemma 8. Assume that $0 \in \omega^{b} \subset \mathbf{R}^{2}$. Let $U=\left\{u \in H^{1}(0,1), u(1)=0\right\}, \widetilde{U}=\left\{u \in \mathcal{C}^{1}[0,1], u(1)=0\right\}$, $V=H_{0}^{2}\left(\omega^{b}\right), \widetilde{V}=\mathcal{C}^{1}\left(\overline{\omega^{b}}\right) \cap H_{0}^{2}\left(\omega^{b}\right), W=\{(u, v) \in U \times V, u(0)=v(0)\}$ and $\widetilde{W}=\{(u, v) \in \widetilde{U} \times \widetilde{V}, u(0)=v(0)\}$. Then $\widetilde{W}$ is dense in $W$. 
Proof. It is clear that $\widetilde{U}$ is dense in $U$ and that $\widetilde{V}$ is dense in $V$. Therefore, for any $(u, v) \in W$, there exists $\left(\bar{u}^{n}, \bar{v}^{n}\right) \in \widetilde{U} \times \widetilde{V}$ such that:

$$
\begin{aligned}
& \bar{u}^{n} \rightarrow u \text { in } H^{1}(0,1) \text { and hence in } \mathcal{C}^{0}[0,1], \\
& \bar{v}^{n} \rightarrow v \text { in } H^{2}\left(\omega^{b}\right) \text { and hence in } \mathcal{C}^{0}\left(\overline{\omega^{b}}\right) .
\end{aligned}
$$

Let $\phi^{1} \in \mathcal{C}^{\infty}[0,1]$ with $\phi^{1}(0)=1, \phi^{1}(1)=0, \phi^{2} \in \mathcal{D}\left(\omega^{b}\right)$ with $\phi^{2}(0)=1$ and let:

$$
\begin{gathered}
u^{n}=\bar{u}^{n}-\left(\bar{u}^{n}(0)-u(0)\right) \phi^{1}, \\
v^{n}=\bar{v}^{n}-\left(\bar{v}^{n}(0)-v(0)\right) \phi^{2} .
\end{gathered}
$$

It is clear that $u^{n} \in \widetilde{U}, v^{n} \in \widetilde{V}$ and $u^{n}(0)=u(0)=v(0)=v^{n}(0)$, so that $\left(u^{n}, v^{n}\right) \in \widetilde{W}$. Moreover:

$$
\left\|u^{n}-\bar{u}^{n}\right\|_{H^{1}(0,1)}=\left|\bar{u}^{n}(0)-u(0)\right|\left\|\phi^{1}\right\|_{H^{1}(0,1)} \rightarrow 0
$$

and hence $u^{n}$ tends to $u$ in $H^{1}(0,1)$. Similarly $v^{n}$ tends to $v$ in $H^{2}\left(\omega^{b}\right)$.

Acknowledgements. The work of Antonio Gaudiello and Jacqueline Mossino was partially supported by the project "Strutture sottili" of the 2004-2006 program "Collaborazioni interuniversitarie internazionali" of the Italian Ministry of Education, University and Research.

\section{REFERENCES}

[1] E. Acerbi, G. Buttazzo and D. Percivale, A variational definition of the strain energy for an elastic string. J. Elasticity 25 (1991) $137-148$.

[2] D.R. Adams and L.I. Hedberg, Fonctions Spaces and Potential Theory. Springer Verlag, Berlin (1996).

[3] G. Anzellotti, S. Baldo and D. Percivale, Dimension reduction in variational problems, asymptotic development in $\Gamma$-convergence and thin structures in elasticity. Asymptot. Anal. 9 (1994) 61-100.

[4] D. Caillerie, Thin elastic and periodic plates. Math. Methods Appl. Sci. 6 (1984) 159-191.

[5] P.G. Ciarlet, Plates and Junctions in Elastic Multi-Structures: An Asymptotic Analysis. Masson, Paris (1990).

[6] P.G. Ciarlet, Mathematical Elasticity, Volume II: Theory of Plates. North-Holland, Amsterdam (1997).

[7] P.G. Ciarlet and P. Destuynder, A justification of the two-dimensional linear plate model. J. Mécanique 18 (1979) 315-344.

[8] A. Cimetière, G. Geymonat, H. Le Dret, A. Raoult, Z. Tutek, Asymptotic theory and analysis for displacements and stress distribution in nonlinear elastic straight slender rods. J. Elasticity 19 (1988) 111-161.

[9] D. Cioranescu and J. Saint Jean Paulin, Homogenization of Reticulated Structures. Springer-Verlag, New York (1999).

[10] M. Dauge and I. Gruais, Asymptotics of arbitrary order for a thin elastic clamped plate, I: Optimal error estimates. Asymptot. Anal. 13 (1996) 167-197.

[11] G. Friesecke, R.D. James and S. Müller, A theorem on geometric rigidity and the derivation of nonlinear plate theory from three-dimensional elasticity. Comm. Pure Appl. Math. 55 (2002) 1461-1506.

[12] G. Friesecke, R.D. James and S. Müller, A hierarchy of plate models derived from nonlinear elasticity by gamma-convergence. Arch. Rat. Mech. Anal. 180 (2006) 183-236.

[13] A. Gaudiello, B. Gustafsson, C. Lefter and J. Mossino, Asymptotic analysis of a class of minimization problems in a thin multidomain. Calc. Var. Part. Diff. Eq. 15 (2002) 181-201.

[14] A. Gaudiello, B. Gustafsson, C. Lefter and J. Mossino, Asymptotic analysis for monotone quasilinear problems in thin multidomains. Diff. Int. Eq. 15 (2002) 623-640.

[15] A. Gaudiello, R. Monneau, J. Mossino, F. Murat and A. Sili, On the junction of elastic plates and beams. C.R. Acad. Sci. Paris Sér. I 335 (2002) 717-722.

[16] A. Gaudiello and E. Zappale, Junction in a thin multidomain for a fourth order problem. M3AS: Math. Models Methods Appl. Sci. 16 (2006) 1887-1918.

[17] I. Gruais, Modélisation de la jonction entre une plaque et une poutre en élasticité linéarisée. RAIRO: Modél. Math. Anal. Numér. 27 (1993) 77-105.

[18] I. Gruais, Modeling of the junction between a plate and a rod in nonlinear elasticity. Asymptotic Anal. 7 (1993) $179-194$. 
[19] V.A. Kozlov, V.G. Ma'zya and A.B. Movchan, Asymptotic representation of elastic fields in a multi-structure. Asymptot. Anal. 11 (1995) 343-415.

[20] H. Le Dret, Problèmes Variationnels dans les Multi-domaines: Modélisation des Jonctions et Applications. Masson, Paris (1991).

[21] H. Le Dret, Convergence of displacements and stresses in linearly elastic slender rods as the thickness goes to zero. Asymptot. Anal. 10 (1995) 367-402.

[22] H. Le Dret and A. Raoult, The nonlinear membrane model as variational limit of nonlinear three-dimensional elasticity. J. Math. Pures Appl. 74 (1995) 549-578.

[23] H. Le Dret and A. Raoult, The membrane shell model in nonlinear elasticity: a variational asymptotic derivation. J. Nonlinear Sci. 6 (1996) 59-84.

[24] R. Monneau, F. Murat and A. Sili, Error estimate for the transition 3d-1d in anisotropic heterogeneous linearized elasticity. To appear.

[25] M.G. Mora and S. Müller, Derivation of the nonlinear bending-torsion theory for inextensible rods by $\Gamma$-convergence. Calc. Var. Part. Diff. Eq. 18 (2003) 287-305.

[26] M.G. Mora and S. Müller, A nonlinear model for inextensible rods as a low energy $\Gamma$-limit of three-dimensional nonlinear elasticity. Ann. Inst. H. Poincaré Anal. Non Linéaire 21 (2004) 271-293.

[27] F. Murat and A. Sili, Comportement asymptotique des solutions du sytème de l'élasticité linéarisée anisotrope hétérogène dans des cylindres minces. C.R. Acad. Sci. Paris Sér. I 328 (1999) 179-184.

[28] F. Murat and A. Sili, Anisotropic, heterogeneous, linearized elasticity problems in thin cylinders. To appear.

[29] O.A. Oleinik, A.S. Shamaev and G.A. Yosifian, Mathematical Problems in Elasticity and Homogenization. North-Holland, Amsterdam (1992).

[30] D. Percivale, Thin elastic beams: the variational approach to St. Venant's problem. Asymptot. Anal. 20 (1999) 39-60.

[31] L. Trabucho and J.M. Viano, Mathematical Modelling of Rods, Handbook of Numerical Analysis 4. North-Holland, Amsterdam (1996). 